\title{
Roles of the HECT-Type Ubiquitin E3 \\ Ligases of the Nedd4 and WWP Subfamilies in Neuronal Development
}

\section{Doctoral Thesis}

\author{
in partial fulfilment of the requirements \\ for the degree "Doctor rerum naturalium" \\ in the Neuroscience Program \\ at the Georg August University Göttingen, \\ Faculty of Biology
}

Submitted by

Hung-En Hsia

Born in

Taipei, Taiwan

May 2015 


\section{Supervisor}

Dr. Hiroshi Kawabe

Department of Molecular Neurobiology, Max Planck Institute of Experimental Medicine, Göttingen

\section{Thesis Committee Members}

Prof. Dr. Nils Brose

Department of Molecular Neurobiology, Max Planck Institute of Experimental Medicine, Göttingen

Dr. Judith Stegmüller

Max Planck Institute of Experimental Medicine, Göttingen

Prof. Dr. Andreas Wodarz

Institute I for Anatomy, University of Cologne, Cologne

\section{Extended Thesis Committee Members}

Dr. Till Marquardt

Developmental Neurobiology, European Neuroscience Institute, Göttingen

Prof. Dr. Andre Fischer

Department of Psychiatry and Psychotherapy, University Medical Center, German Center for Neurodegenerative Diseases, Göttingen

Prof. Dr. Thomas Dresbach

Department of Anatomy and Embryology, Göttingen University Medical School Center of Anatomy, Göttingen

\section{Date of Oral Examination}

$20^{\text {th }}$ October 2014 


\section{Declaration}

I hereby declare that this thesis has been written independently, with no other aids than those cited.

Hung-En Hsia 


\section{Acknowledgements}

I owe many thanks to people who support me throughout my doctoral study. First of all, I would like to express my sincere gratitude to my supervisor, Dr. Hiroshi Kawabe, for including me as a member of his group and introducing me the projects. I am grateful for his encouragement, guidance, training in experimental skills, and for urging me to achieve better after I have already done my best. I am indebted to Prof. Dr. Nils Brose, head of the Molecular Neurobiology Department, for embracing me as a colleague in his department, for his constant support, and his critical scientific input. I would also like to thank both of them for proofreading this thesis.

I am also grateful to Dr. Judith Stegmüller and Prof. Dr. Andreas Wodarz for being my thesis committee members to provide valuable input on the progress of my work.

It has been my honor to work with Mika Kishimoto-Suga, Michiko Takeda, Mateusz Ambrozkiewicz, Bekir Altas, and Manuela Schwark, who are members of the Kawabe Group. I would also like to thank members of the Ubiquitin/SUMO Subgroup. We had many fruitful scientific discussions. Many of my works would not have been done without their input. I am deeply grateful to Bernd Hesse-Nießen, Klaus-Peter Hellmann, Ivonne Thanhäuser, Dayana Schwerdtfeger, Christiane Harenberg, and Fritz Benseler for their excellent technical assistance, and to the staff of the animal facility at the Max Planck Institute for Experimental Medicine for maintenance of the mouse colony. I would also like to acknowledge Jennifer Day for proofreading this thesis, and to all my friends and colleagues in the Molecular Neurobiology Department for offering me support, providing me with troubleshooting tips, and creating a stimulating scientific and social environment in the Brose Department.

I had the privilege of working with my collaborators on the PTEN/Nedd4 paper. The PTEN/Nedd4 paper would have been markedly poorer without their helps. I am grateful to all of them, especially to Dr. Julien Courchet and 
Dr. Franck Polleux for advising me on how to study the axonal morphology in mouse brains and providing me with the macro to analyze axonal branching in vivo; and to Rossella Luca and Dr. Claudia Bagni for helping me with the ribosome profiling experiments.

I would also like to thank Dr. Olaf Jahn and his Proteomics Group for the support in mass spectrometric analysis to identify WWP1 binding proteins.

The IMPRS Neuroscience Program and all of its constituents have provided me the ideal framework for academic achievements in Göttingen. I am grateful to Sandra Drube and Prof. Michael Hörner, who help me with the administrative matters throughout the course of my doctoral study.

I would also like to thank my friends, who share some great moments with me and let me know I am not alone during some desperate moments.

Last but not least, I am indebted to my family: to my parents, who always give me their greatest support without asking for return; to my elder sister, who encourages me though she is also struggling with her doctoral study; and to my maternal grandmother in heaven, who raises me and uses her life-long story to show me how a woman can be tough, yet remain tender. I would like to dedicate this work to her. 


\section{Summary}

Protein ubiquitination is a core regulatory principle in neuronal development. In this study, I used brain specific KO mice to investigate the roles of several HECT-type Nedd4 superfamily E3 ligases, i.e. Nedd4-1, Nedd4-2, WWP1, and WWP2, during brain development. I show that Nedd4-1 and Nedd4-2 are required for neuronal axonal growth and branching in the mouse central nervous system. Previously published data indicated that the lipid phosphatase PTEN may be a relevant substrate of Nedd4-1 and Nedd4-2. However, I show that aberrant PTEN ubiquitination is not involved in the impaired axon growth upon deletion of Nedd4-1 and Nedd4-2. Rather, PTEN limits Nedd4-1 expression at the translational level by modulating the activity of mTORC1, a protein complex that controls protein synthesis and cell growth. I further show that Nedd4-1 is one of the major targets of PTENmTORC1 signaling in the control of neurite growth. In addition, I identify the deubiquitinase Usp9x as a binding partner of WWP1 and WWP2. WWP1 and WWP2 may counteract the function of Usp9x by interacting with Nuak1 and Nuak2, protein kinases that can be deubiquitinated and activated by Usp9x. Moreover, I show that Cdk5, a protein kinase with multiple roles in the nervous system, is ubiquitinated by WWP1 and WWP2 in vivo via a K63-linked polyubiquitin chain. Such ubiquitination of Cdk5 by WWP1 and WWP2 may alter Cdk5 activation or subcellular localization and may thus affect multiple cellular processes during brain development. 


\section{Abbreviations}

\begin{tabular}{|c|c|}
\hline AMP & Adenosine-5'-monophosphate \\
\hline ATP & Adenosine-5'-triphosphate \\
\hline BDNF & Brain-derived neurotrophic factor \\
\hline BSA & Bovine serum albumin \\
\hline Cdk5 & Cyclin-dependent kinase 5 \\
\hline C. elegans & Caernorhabditis elegans \\
\hline Cre & Cre recombinase \\
\hline DIV & Days in vitro \\
\hline DMEM & Dulbecco's modified Eagle's medium \\
\hline DNA & Deoxyribonucleic acid \\
\hline dNTPs & Deoxynucleosides-5'-triphosphate \\
\hline DTT & Dithiothreitol \\
\hline DUB & Deubiquitinase \\
\hline E. coli & Escherichia coli \\
\hline E1 & Ubiquitin activating emzyme \\
\hline E2 & Ubiquitin conjugating emzyme \\
\hline E3 & Ubiquitin ligase \\
\hline EGFP & Enhanced green fluorescent protein \\
\hline FCS & Fetal calf serum \\
\hline GTP & Guanosine-5'-triphosphate \\
\hline GST & Glutathione S-transferase \\
\hline HECT & Homologous-to-E6-AP-C-terminus \\
\hline IB & Immunoblotting \\
\hline IP & Immunoprecipitation \\
\hline KD & Knockdown \\
\hline $\mathrm{kDa}$ & Kilo Dalton \\
\hline $\mathrm{KO}$ & Knockout \\
\hline MEF & Mouse embryonic fibroblast \\
\hline mRNA & Messenger ribonucleic acid \\
\hline mRNP & Messenger ribonucleoprotein \\
\hline mTORC1 & $\begin{array}{l}\text { Serine/threonine protein kinase mammalian target of } \\
\text { rapamycin complex } 1\end{array}$ \\
\hline MZ & Marginal zone \\
\hline Nedd4 & $\begin{array}{l}\text { Neuronal precursor cell-expressed developmentally down- } \\
\text { regulated } 4\end{array}$ \\
\hline $\mathrm{NEX}-\mathrm{N} 1 / 2^{\mathrm{f} / \mathrm{f}}$ & NEX-Cre;Nedd4-1/f/f $;$ Nedd4 $4-2^{f / f}$ \\
\hline PBS & Phosphate buffered saline \\
\hline PI3K & Phosphoinositide 3-kinase \\
\hline Ptdlns $P_{2}$ & Phosphatidylinositol-4,5-bisphosphate \\
\hline PtdlnsP $_{3}$ & Phosphatidylinositol-3,4,5-trisphosphate \\
\hline PTEN & Phosphatase and tensin homolog \\
\hline RGC & Radial glial cell \\
\hline RING & Really-Interesting-New-Gene \\
\hline RT & Room temperature \\
\hline SDS-PAGE & e-polyacrylamide gel electrophoresis \\
\hline
\end{tabular}


SEM

$S V Z$

VZ

WT

WWP
Standard error of the mean

Subventricular zone

Ventricular zone

Wild-type

WW domain-containing protein 


\section{Table of Contents}

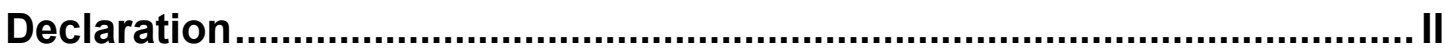

Acknowledgements .....................................................................................

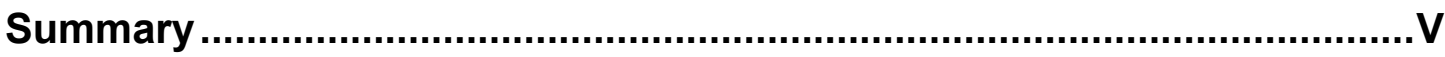

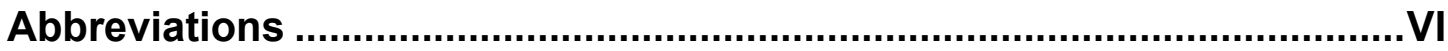

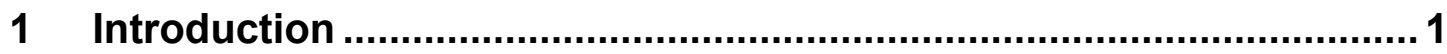

1.1 Nerve Cells Development in the Murine Cerebral Cortex ............ 1

1.1.1 Neurogenesis ....................................................................... 1

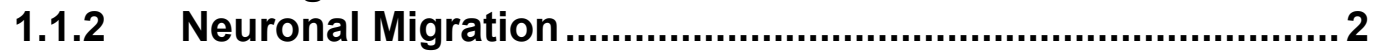

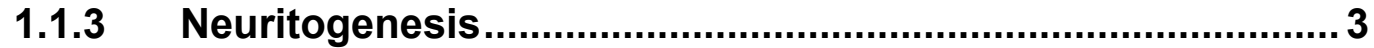

1.1.4 Synaptogenesis ................................................................ 4

1.1.5 Perspectives on Studies of Neuronal Development ............. 4

1.2 Ubiquitination........................................................................ 5

1.2.1 Systematic Screening for Protein Substrates of E3 LigaseSpecific Ubiquitination.......................................................... 8

1.3 Roles of the HECT-Type Nedd4 Superfamily E3 Ligases in Neuronal Development..............................................................11

1.3.1 Nedd4-1 and Nedd4-2 ......................................................12

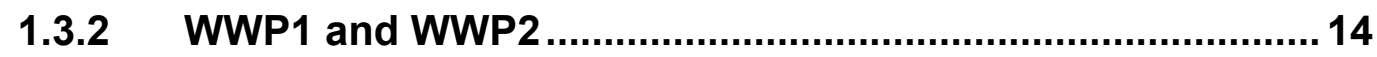

1.4 Roles of PTEN in Neuronal Development...................................15

1.5 Roles of Cdk5 in Neuronal Development ...................................17

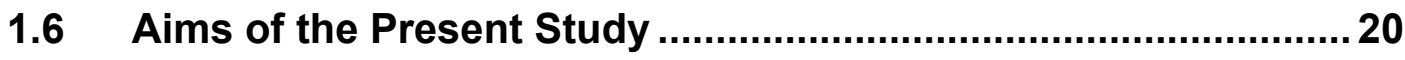

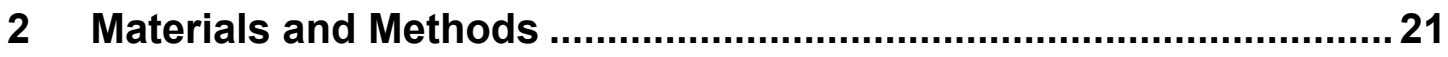

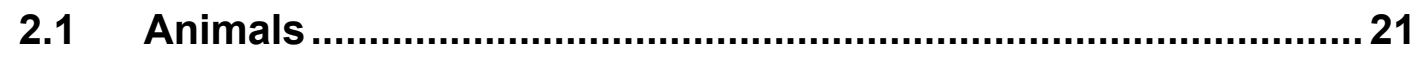

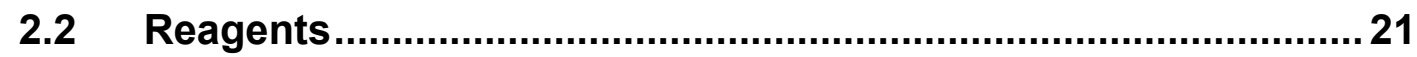

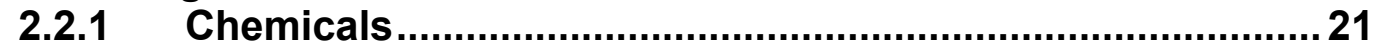

2.2.2 Enzymes from Commercial Sources ................................23

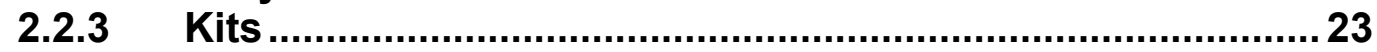

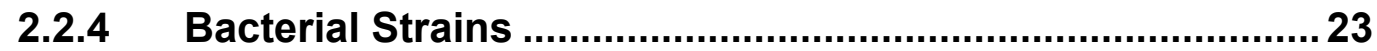

2.2.5 Vector Plasmids ........................................................... 23

2.2.6 Oligonucleotides.............................................................25

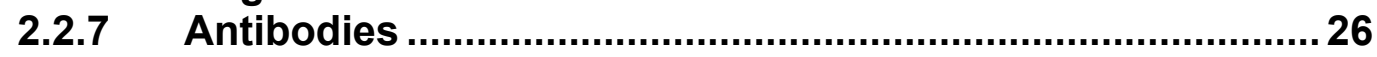

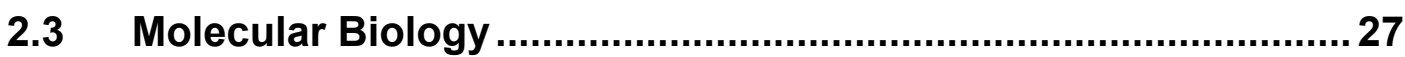

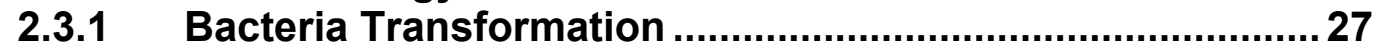

2.3.2 Plasmid DNA preparation ................................................28

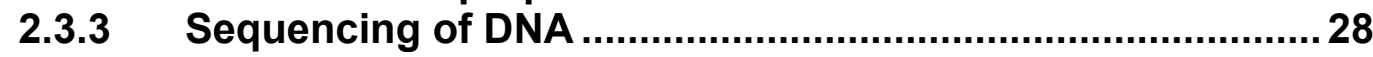

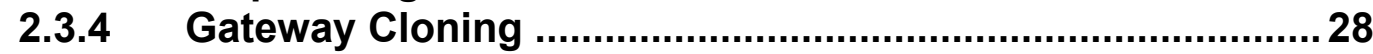

2.3.5 DNA Digestion with Restriction Endonucleases .................29 


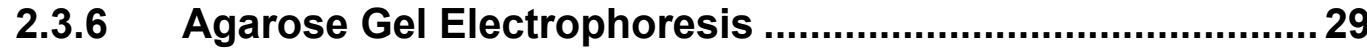

2.3.7 Purification of DNA Fragments ......................................29

2.3.8 De-phosphorylation of 5'-DNA Ends..................................29

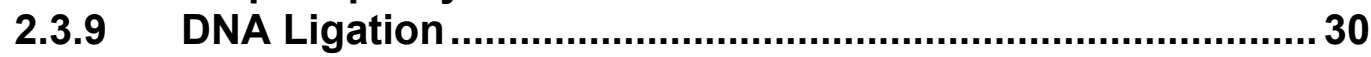

2.3.10 Polymerase Chain Reaction (PCR) ……............................ 30

2.3.11 Site-Directed Mutagenesis ...............................................30

2.3.12 Subcloning using the TOPO Cloning Kit..............................31

2.3.13 RNA Preparation ................................................................. 31

2.3.14 Real Time Quantitative-PCR (RT-qPCR) ..............................32

2.3.15 Cloning Strategies for Constructs Generated in This Study

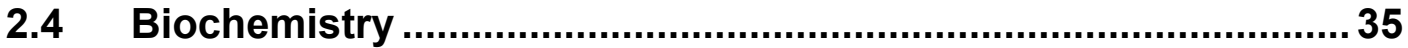

2.4.1 Determination of Protein Concentration ............................35

2.4.2 Sodium dodecyl sulfate polyacrylamide gel

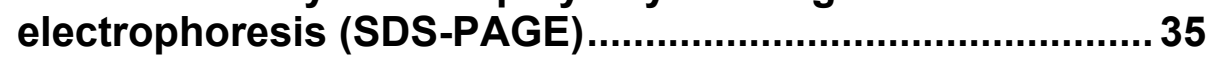

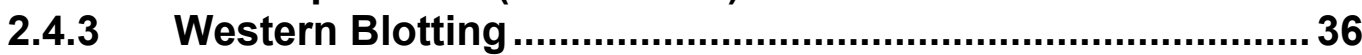

2.4.4 Purification of Recombinant GST-Fusion Proteins .............. 37

2.4.5 Affinity Purification of GST-WWP1 Binding Proteins...........38

2.4.6 Protein Identification by Mass Spectrometry …...................39

2.4.7 In Vitro Binding Assay ........................................................

2.4.8 In Vitro Ubiquitination Assay............................................. 40

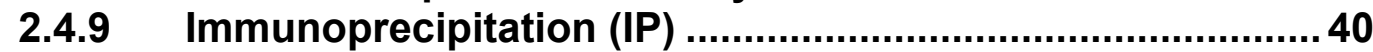

2.4.10 In Vivo Ubiquitination Assay ............................................41

2.4.11 PTEN Phosphatase Activity Assay …….............................. 41

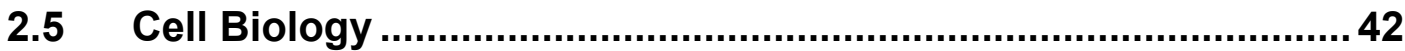

2.5.1 Culture Media and Solutions ...............................................42

2.5.2 Primary Mouse Hippocampal Culture Preparation...............43

2.5.3 Transfection of Primary Hippocampal Culture ......................43

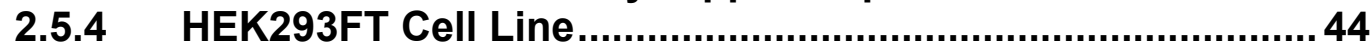

2.5.5 Lentivirus Preparation ........................................................ 44

2.5.6 Immunocytochemistry (ICC) ........................................... 44

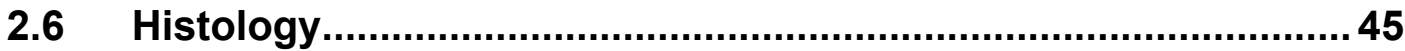

2.6.1 In Utero Electroporation..................................................... 45

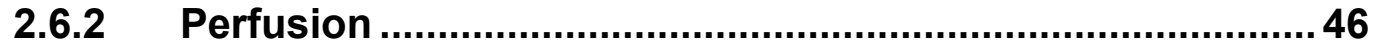

2.6.3 Immunohistochemistry (IHC) ........................................... 46

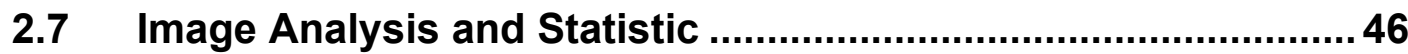

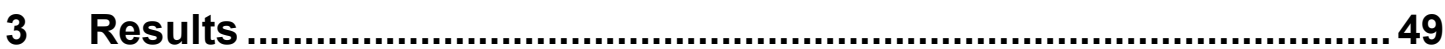

3.1 Ubiquitin E3 Ligase Nedd4-1 Acts as a Downstream Target of PI3K/PTEN-mTORC1 Signaling to Promote Neurite Growth...... 49

3.1.1 KO of Nedd4-1 and Nedd4-2 Causes Defects in Axonal

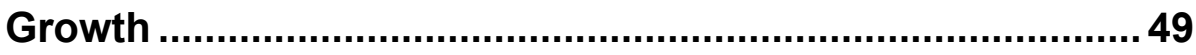

3.1.2 PTEN is not Targeted by Nedd4-1 or Nedd4-2 for Proteosomal Degradation ..................................................55

3.1.3 PTEN Neither Poly- Nor Mono-ubiquitinated by Nedd41/Nedd4-2 in Developing Mammalian Neurons. 
3.1.4 PTEN Acts as a Negative Regulator of Nedd4-1 Expression at the Translational Level

3.1.5 Nedd4-1 is a Major Target of mTORC1 Signaling in Neurite Development. 64

3.1.6 Pathways Operating Parallel to the PI3K/PTEN-mTORC1Nedd4-1 Signaling Regulates Neurite Growth and Polarity Formation

3.1.7 Working Model

3.2 Roles of the E3 Ligases WWP1 and WWP2 during Mammalian

Brain Development ............................................................... 71

3.2.1 Identification of Binding Partners of WWP1 ......................71

3.2.2 Proteomic Based Screening of Ubiquitination Substrates of WWP1 and WWP2 in Mouse Brains .................................74

3.2.3 Cdk5 is a Physiological Substrate of WWP1 and WWP2 ....76

3.2.4 Regulation of Cdk5 by WWP1/WWP2 Mediated

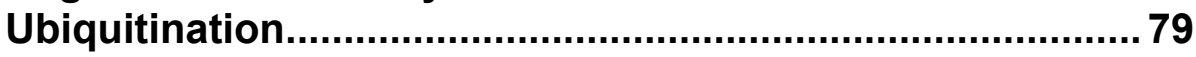

4 Discussion 82

4.1 PTEN is Not a Relevant Substrate of Mammalian Nedd4 Family E3 Ligases in the Regulation of Neurite Development

4.2 PTEN Negatively Regulates Nedd4-1 Expression at the Translational Level

4.2.1 Nedd4-1 is a Major Target of the PI3K/PTEN-mTORC1

Signaling in Neurite Development 85

4.2.2 Possible Roles of Nedd4-1 in Insulin-Like Growth Factor-1 (IGF-1) Signaling 86

4.3 Opposing Roles of Usp9x and WWP1/WWP2 in the Development of Mammalian Neurons. 88

4.4 Regulation of Cdk5 by WWP1/WWP2-Mediated Ubiquitination. 90

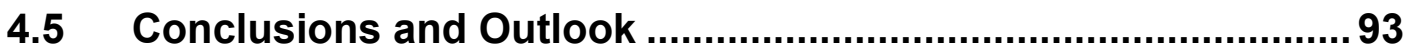

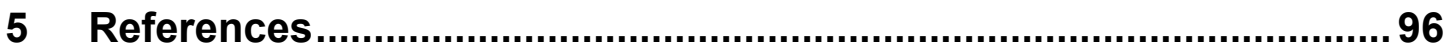

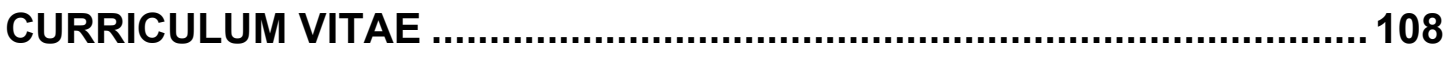

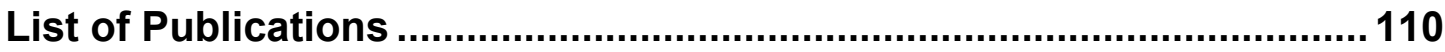




\section{Introduction}

\subsection{Nerve Cells Development in the Murine Cerebral Cortex}

As the center of nervous system, the brain is the most complex mammalian organ with the cerebrum occupying the largest part. The murine cerebral cortex is comprised of billions of neurons, each of which communicates with others through thousands of synapses (HerculanoHouzel et al., 2006). The resulting neuronal network contains trillions of synapses in a highly ordered manner and is responsible for cognitive functions (e.g. learning and memory), perceptions (e.g. hearing, vision, taste, olfaction, and somatic sensory), movements, and consciousness. The formation of this network is dependent on the development of neurons, which are specialized cells that allow the directional transfer of signals through their two types of protrusions: dendrites and axons. A neuron projects multiple dendrites to receive synaptic inputs, and a single extended axon to send synaptic outputs. In the mammalian cerebral cortex, the development of neurons is characterized by four distinctive but partially overlapping stages: first, neuronal progenitor cells proliferate and differentiate into neurons (neurogenesis); second, newly generated neurons move toward their final destination (migration); third, neurons project dendrites and axons (neuritogenesis); and fourth, synapses are formed between dendrites and axons (synaptogenesis; Figure 1-1; reviewed by Parrish et al., 2007).

\subsubsection{Neurogenesis}

Three types of neurogenic progenitor cells reside in the developing neocortex: neuroepithelial cells, radial glial cells (RGCs), and intermediate progenitors. Neuroepithelial cells undergo symmetric cell division at the ventricular zone (VZ) to expand its population and subsequently generate neurons by asymmetric division. RGCs arise from neuroepithelial cells at the apical surface of the VZ. They also proliferate via symmetric cell division and generate neurons and intermediate progenitors by asymmetric cell division. 
Intermediate progenitors divide only once to give rise to two postmitotic neurons in the subventricular zone (SVZ; Figure 1-1A; reviewed by Gotz and Huttner, 2005).

\subsubsection{Neuronal Migration}

The newly generated neurons in the neocortex migrate radially along the projections of RGCs from the VZ or the SVZ toward the cortical plate. Neurons generated in the early phase of neurogenesis migrate first and settle down at the marginal zone $(\mathrm{MZ})$, whereas neurons generated in later phases overtake the existing neurons and migrate further (neurons $i$ and ii in Figure $1-1 B)$. Following this 'inside-out' fashion of neuronal migration, the deepest layer of the neocortex is established first (reviewed by Lambert de Rouvroit and Goffinet, 2001).

Migration of neurons requires orchestrated activities of multiple cellular components, with the centrosome-derived cytoplasmic microtubules playing a central role. Steered by extracellular guidance cues, locomotion of the neuron is carried out by three successive but interdependent cellular modes: extension of the leading process, transition of the nucleus (nucleokinesis), and retraction of the trailing process. The centrosome of migrating neuron is positioned in front of the nucleus towards the direction of migration, a configuration called 'nucleus-centrosome coupling' that is crucial for nucleokinesis. After the leading process elongates along the projections of RGCs, the centrosome advances into the leading process and uncouples from the nucleus. The microtubule minus-end-directed motor proteins such as dynein and its associated proteins provide the pulling forces for nucleokinesis to restore the nucleus-centrosome coupling. Upon receiving the Reelin signal secreted by the Cajal-Retzius cells at the $M Z$, neurons detach from the RGC and stop migrating (reviewed by Frotscher, 1998; Honda et al., 2011). 


\subsubsection{Neuritogenesis}

Neuritogenesis starts during migration (Figure 1-1C). Individual migrating neurons extend two main processes toward the $M Z$ and the $V Z$, which later differentiate into dendrites and axons, respectively. At the initial stage of neuritogenesis, several signaling molecules such as CAMP, PI3K, and PAR6, showed polarized distribution in one of the neurites, contributing to the rearrangements of cytoskeleton in this specific neurite. Such signaling drives the extension of a single neurite, which will obtain the function of an axon later in development (reviewed by Arimura and Kaibuchi, 2007; Barnes and Polleux, 2009; Cheng and Poo, 2012).

The developing axons need to be guided to the defined targets. This process is controlled by extracellular cues including chemoattractants and chemorepellants, such as Notch, semaphorins, ephrins, reelin and neurotrophins, in combination with the corresponding receptors expressed at the growth cones of extending axons. Activation of these receptors triggers intracellular signaling cascades, leading to rearrangements of cytoskeleton and asymmetric membrane trafficking that drive the axonal branching and growth cone turning. Therefore, the extending axons are allowed to navigate the environment to find the correct targets (reviewed by O'Donnell et al., 2009; Tojima et al., 2011).

Dendrites differ from axons in many morphological and functional aspects. Accordingly, morphogenesis of dendrites involves some different underlying mechanisms as that of axons. Intrinsic regulars such as transcriptional factors contribute to the neuronal type-specific dendritic patterns. Neurogenin-2, for example, is a basic helix-loop-helix (bHLH) transcription factor that has a crucial role in the specification of dendritic morphology of pyramidal neurons in the neocortex. Other transcription factors such as CREST and CREB respond to the $\mathrm{Ca}^{2+}$-dependent intracellular cascades and regulate the activity-dependent dendritic outgrowth. Importantly, extracellular cues that operate for axonal guidance, on the other hand, play roles in regulating the arborization of dendrites 
(reviewed by Jan and Jan, 2010; Puram and Bonni, 2013). Therefore, dendrites establish their overall arborization patterns through a combination of intrinsic and extrinsic mechanisms.

\subsubsection{Synaptogenesis}

Synaptogenesis takes place mostly postnatally, which is in line with the fact that this process is strongly influenced by the activity within the developing neuronal network (Figure 1-1D). The initial contact between an axonal growth cone and a target neuron is mediated by various synaptic adhesion proteins, which interact with each other in a trans configuration in an isoform-specific manner to allow for the cell-type specificity of synaptogenesis. Upon the establishment of nascent synaptic contacts, synaptic organizing signals contributed by the adhesion proteins then coordinate the subsequent maturation process, during which hundreds of specific proteins are sorted to the pre- and postsynaptic compartments. In the mature synapse, presynaptic neurotransmitter releasing sites are opposed to the postsynaptic signal-receiving compartment, or the postsynaptic density. The pre- and post-synapse contains distinct sets of adhesion and scaffolding proteins that are required for equipping the synapse with the proper presynaptic neurotransmitter releasing machinery and postsynaptic receptors (reviewed by Brose, 1999; Garner et al., 2002; Siddiqui and Craig, 2011; Waites et al., 2005; Yamagata et al., 2003).

\subsubsection{Perspectives on Studies of Neuronal Development}

Dysregulation of any of these four steps of nerve cell development may lead to neurological or psychiatric disorders, such as Rett syndrome, Down's syndrome, Fragile $X$ syndrome, or autism spectrum disorders (reviewed by Bagni and Greenough, 2005; Dierssen and Ramakers, 2006; Kaufmann and Moser, 2000; Pardo and Eberhart, 2007). Studies of the underlying molecular mechanisms of nerve cell development, therefore, is important for understanding the pathology of these disorders as well as normal brain 
functions. In the past three decades, it has been established that the four key steps of nerve cell development are coordinated by defined signaling pathways mediated by protein and lipid phosphorylation, $\mathrm{Ca}^{2+}$ signaling, and protein ubiquitination (reviewed by Arimura and Kaibuchi, 2007; DiAntonio and Hicke, 2004; Kawabe and Brose, 2011; Takemoto-Kimura et al., 2010). However, if and how these pathways interact and contribute to the neuronal developing processes remains largely unexplored.

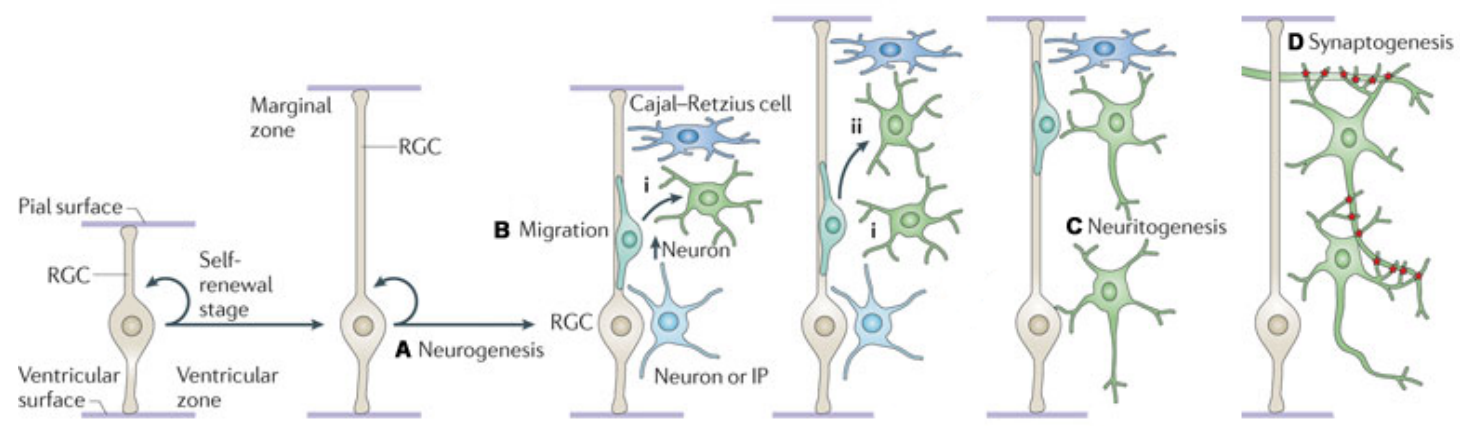

Figure 1-1. Key Stages of Neuronal Development in the Rodent Neocortex

In the ventricular zone, neuroepithelial cells undergo cell division to expand their population and generate radial glia cells (RGCs). (A) RGCs proliferate via symmetric cell division to generate neurons or intermediate progenitors (IP) by asymmetric cell division. (B) An IP divides only once at the subventricular zone to generate two neurons, which migrate vertically along the radial projections of RGCs toward the cortical plate. External cues secreted by Cajal-Retzius cells cause the detachment of neurons from the radial projection and stop the neuronal migration. Note that neurons migrate towards the cortical plate in an inside-out manner (neurons $\mathrm{i}$ and ii), so that the deepest layer of the cortex is established first. (C) During migration, neurons start extending neurites, which later differentiate into dendrites and axons. (D) Dendrites and axons further extend and branch to form initial contacts. Synaptic adhesion proteins then coordinate the recruitments of preand post-synaptic components for the formation of synapses. Adapted by permission from Macmillan Publishers Ltd: Nat. Rev. Neurosci. (Kawabe and Brose, 2011), Copyright, 2011.

\subsection{Ubiquitination}

Ubiquitination is a protein post-translational modification that involves the conjugation of one or more ubiquitin moieties to substrate proteins, and 
thereby regulates the stability or function of the substrate proteins. This process is carried out by a sequential reaction catalyzed by three classes of enzymes: ubiquitin-activating enzymes (E1), ubiquitin-conjugating enzymes (E2), and ubiquitin ligases (E3). An E1 forms a high-energy thioester bond with an ubiquitin at the expense of ATP. The activated ubiquitin conjugated on the E1 is then transferred to an E2, which directly interacts with an E3. The E3 then mediates the conjugation of ubiquitin onto substrate proteins. E3 ligases determine the substrate specificity by interacting with the substrate proteins, and they belong to either of the two families based on their domain structures: Really Interesting New Gene type ligases (RING finger-type E3 ligases) and Homologous to E6-AP Carboxyl-Terminus type ligases (HECTtype E3 ligases). RING finger-type E3 ligases transfer the ubiquitin moiety from E2 to substrates directly, whereas HECT-type E3 ligases first covalently bind to the ubiquitin moiety at a cysteine residue in their HECT domain and then transfer it to the substrates. In contrast to E1 and E2 enzymes, E3s have higher diversity in higher-order animals. Approximately 600 genes encode E3s in the human genome. Owing to their diversity, E3 ligases are considered to be the main determinant of the substrate specificity in the protein ubiquitination processes (Figure 1-2A; reviewed by Komander and Rape, 2012; Welchman et al., 2005).

A single 76-amino acid long ubiquitin protein has seven lysine residues, all of which can be used for the formation of polyubiquitin chains. Lysine 48linked (K48-linked) polyubiquitin chains, in which the carboxyl-terminal glycine residue of one ubiquitin moiety is linked to the K48 of another ubiquitin moiety, direct protein degradation by the $26 \mathrm{~S}$ proteasome. More recent studies have revealed that monoubiquitination and ubiquitin chains conjugated through the other six lysine residues also play important roles in the regulation of numerous cellular processes. K63-linked polyubiquitin chains, for example, have been reported to control DNA repair, NFKB activation, translational regulation, and endocytosis. Monoubiquitination or multi-monoubiquitination of proteins, on the other hand, has been shown to 
regulate endocytosis and trafficking of plasma membrane proteins (Figure 12B; reviewed by Ikeda and Dikic, 2008).

Similar to phosphorylation or many of other protein posttranslational modifications, ubiquitination is a reversible process and can be reversed by the action of deubiquitinases (DUBs). Such dynamic regulation allows the protein ubiquitination procedure responding to various signals to regulate the fast-changing cellular processes.

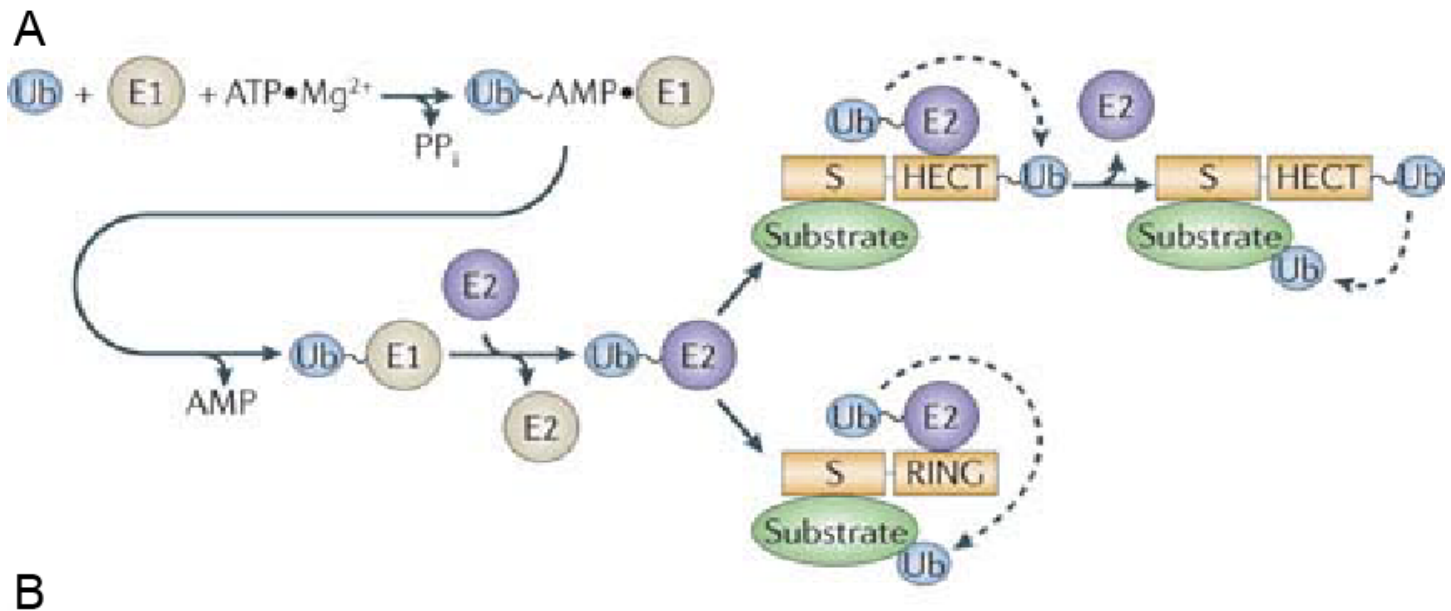

Polyubiquitylation (Lys 11-or Lys48-linked)

(Substrate) (Ub) Ub $\longrightarrow$ Proteasomal degradation

Polyubiquitylation (Lys63-linked)

Substrate

UbUbUbUb $\longrightarrow$ Regulation of protein functions and interactions

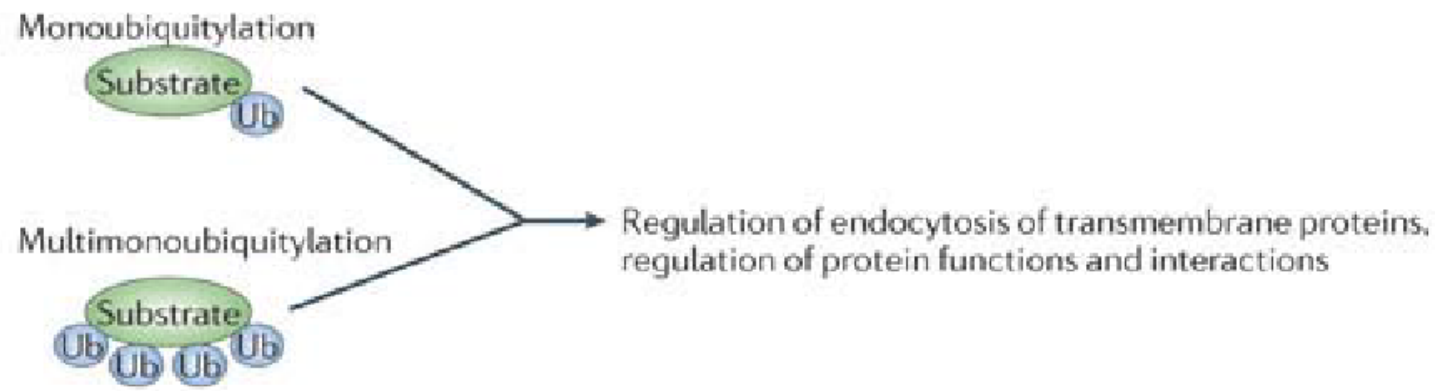

Figure 1-2. The Protein Ubiquitination Pathway

(A) Protein ubiquitination is a sequential reaction catalyzed by three classes of enzymes. An ubiquitin-activating enzyme (E1) forms a thioester bond with 
a free ubiquitin moiety at the expense of ATP. The activated ubiquitin is subsequently transferred to an ubiquitin-conjugating enzyme (E2), which is in turn recognized by an ubiquitin ligase (E3). HECT-type E3 ligases covalently bind the activated ubiquitin received from E2s on a cysteine residue in the HECT domain, and subsequently transfer the ubiquitin to a lysine residue on the substrate protein that is recognized by the substrate recognition domain (S) of the E3 ligase. On the other hand, RING finger-type E3 ligases directly transfer the activated ubiquitin from E2 enzymes to substrate proteins. $(B)$ Functional consequences of protein Ubiquitination. Proteins conjugated with K48-linked polyubiquitin chains or K11-linked polyubiquitin chains are directly recognized by the $26 \mathrm{~S}$ proteasome and targeted for degradation. Proteins conjugated with K63-linked polyubiquitin chains may have altered functions. Monoubiquitination or multi-monoubiquitination of proteins regulates the function or endocytosis of proteins. PPi, pyrophosphate; Ub, ubiquitin. Adapted by permission from Macmillan Publishers Ltd: Nat. Rev. Neurosci. (Kawabe and Brose, 2011), Copyright, 2011.

\subsubsection{Systematic Screening for Protein Substrates of E3 Ligase- Specific Ubiquitination}

Despite the importance of protein ubiquitination in the regulation of multiple cellular processes, the comprehensive identification of specific substrates of individual E3 ligases has remained a challenge. Methods for globally quantitative assessment of protein amounts or protein stability, such as SILAC (Krüger et al., 2008), global protein stability profiling (Yen et al., 2008), or iTRAQ labeling (Wiese et al., 2007) may give hints for substrate proteins that are modified through K48-linked polyubiquitination. However, recent studies have revealed the importance of monoubiquitination and ubiquitin chains conjugated through the other six lysine residues, which may not regulate the stability of substrate proteins. In addition, the alterations in protein amounts or stability detected using those methods may be a secondary effect rather than a direct consequence of protein ubiquitination. Therefore, direct assessment of ubiquitin-modified proteomes, or ubiquitomes, is more informative for the identification of ubiquitination substrates.

Systematic identification of ubiquitination substrates can be achieved by the application of protein microarrays. Using purified recombinant E3 ligases 
on protein microarrays, one can perform binding assays to screen for binding proteins, or perform in vitro ubiquitination assays using fluorescently-labeled ubiquitin to screen for potential protein substrates. Experiments using protein microarrays and the recombinant E3 ligase Rsp5, a yeast orthologue of vertebrate Nedd4, identified several previously known as well as putative novel binding partners and ubiquitination substrates of Rsp5, indicating the validity of this method (Gupta et al., 2007; Kus et al., 2005).

Purification of the ubiquitomes from cells, followed by identification of purified proteins using mass spectrometric analyses can also be applied to identify ubiquitination substrates. However, protein deubiquitination mediated by DUBs is a confounding factor, whose circumvention requires substantial enrichment of ubiquitination and low complicity samples. One of the approaches to circumvent the problems posed by DUBs is to overexpress

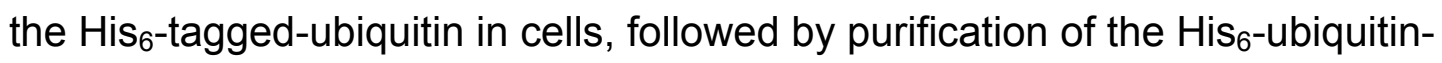
modified ubiquitomes using Ni-NTA chromatography under denaturing conditions (Peng et al., 2003). The reliability of this method, however, is questionable as overexpression of the $\mathrm{His}_{6}$-ubiquitin can result in artificial offtarget ubiquitination.

The development of tandem-repeated ubiquitin-binding entities (TUBEs) offers a solution to purify endogenous ubiquitinated proteins while at the same time protecting them from being deubiquitinated by DUBs. As compared to single ubiquitin associated domain (UBAs), TUBEs show higher affinity to K48- or K63-lined tetra-ubiquitin, and thus can be useful to efficiently purify endogenous K48-linked or K63-linked polyubiquitinated proteins from cells (Hjerpe et al., 2009). The purification and identification of monoubiquitinated proteins using TUBEs, nevertheless, is not an ideal approach given the rather weak affinities between TUBEs and monoubiquitinated proteins.

Further advances in purification of endogenous ubiquitomes involved the enrichment of ubiquitomes at the peptide level instead of at the protein level. Following trypsin digestion of proteins, the resulting peptides containing 
di-glycine ubiquitin remnants left on the lysine residues (K-ع-GG) can be immunopurified using a monoclonal antibody specific to K-E-GG. This approach reduces sample complexity for mass spectrometric analysis, resulting in an increased signal-to-noise ratio and the identification of a

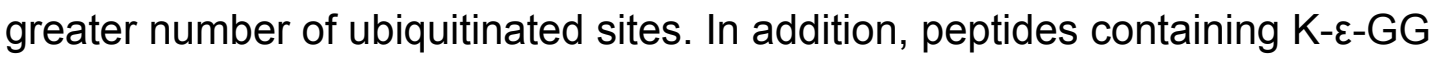
are contributed by endogenous E3 ligases, leading to more reliable identification of physiological substrates (Kim et al., 2011; Figure 1-3).

In summary, various strategies have been developed to systematically identify the ubiquitination substrates of a given E3 ligase, and each of them has advantages and disadvantages. Combining more than one strategy may increase the validity of identification results. In addition, methods such as in vitro ubiquitination assays or in vivo ubiquitination assays can be applied to validate the screening results.
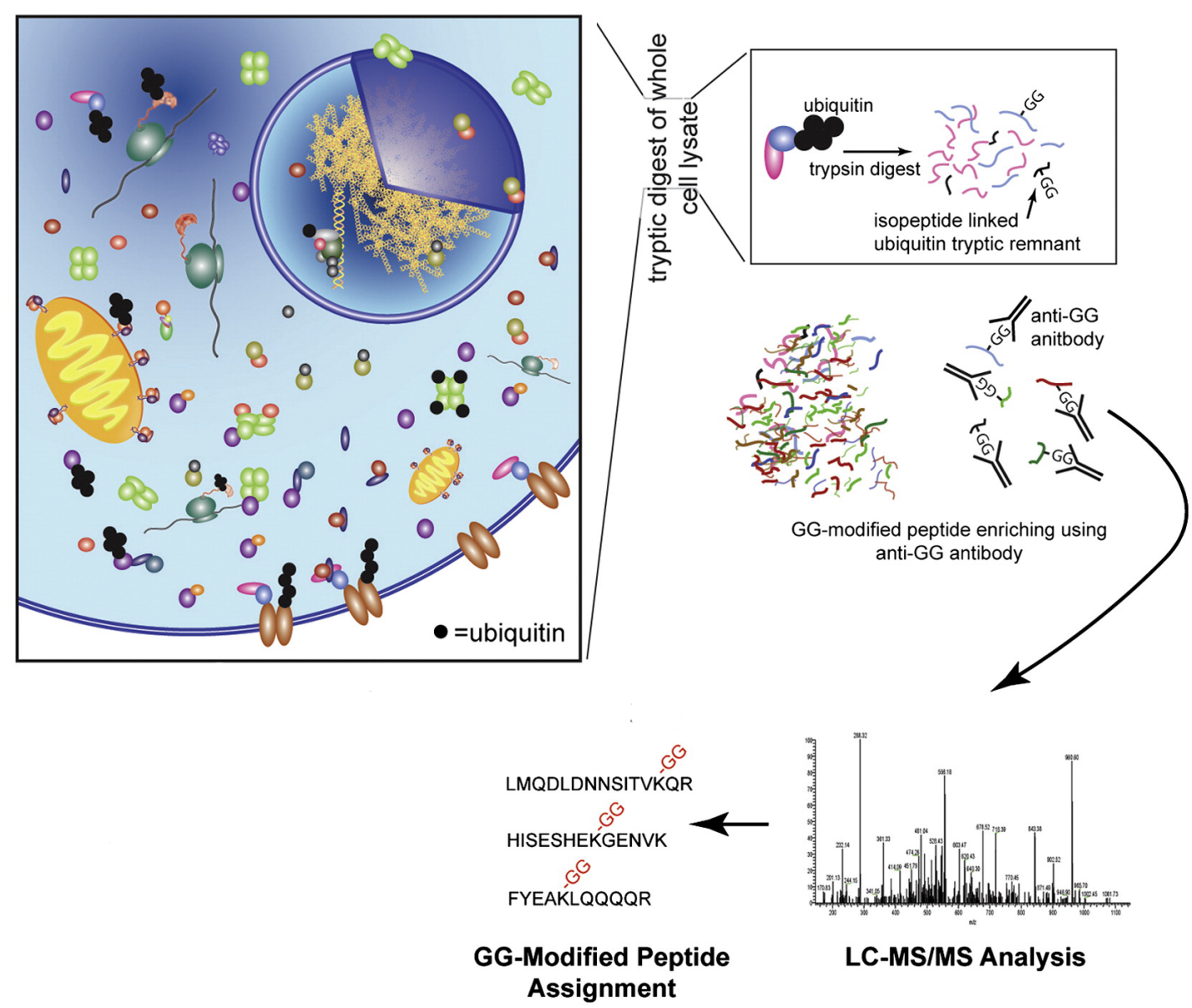

LC-MS/MS Analysis 
Figure 1-3. Quantitative Assessment of the Ubiquitin-Modified Proteome at the Peptide Level

Cell lysates containing ubiquitinated proteins were trypsinized, and the resulting peptides containing di-glycine ubiquitin remnants (GG) left on the lysine residues were immunopurified with an anti-GG antibody. The GGmodified peptides were then subjected to the liquid chromatography-tandem mass spectrometric (LC-MS/MS) analyses for identification and quantification. The identified GG-modified peptides were assigned to the corresponding proteins. Adapted from (Kim et al., 2011) with permission from Elsevier, Copyright, 2011.

\subsection{Roles of the HECT-Type Nedd4 Superfamily E3 Ligases in Neuronal Development}

The murine Neuronal precursor cell-expressed Developmentally Downregulated 4 (Nedd4) superfamily belongs to the HECT type E3 ligases family and contains eight members that share similar domain structures, which are consisted of an $\mathrm{N}$-terminal C2 domain, two to four tryptophan-rich WW domains at the central region, and a C-terminal HECT domain (reviewed by Rotin and Kumar, 2009). The $\mathrm{C} 2$ domain acts as a $\mathrm{Ca}^{2+}$ sensor that mediates the $\mathrm{Ca}^{2+}$-dependent membrane targeting of the ligase as well as proteinprotein interactions, including the intramolecular interaction with the HECT domain that leads to autoinhibition of the ligase activity (Wang et al., 2010). WW domains recognize and bind to the proline-rich motif of the substrates (e.g. PPXY or LPXY, where ' $x$ ' stand for any amino acids), and thus are critical for substrate proteins recognition. The HECT domain is essential for ubiquitination of substrates by forming a thioester intermediate with an ubiquitin moiety at a conserved cysteine residue in the C-terminal region, and then transfers the ubiquitin to substrates (Figure 1-4; reviewed by Rotin and Kumar, 2009). 


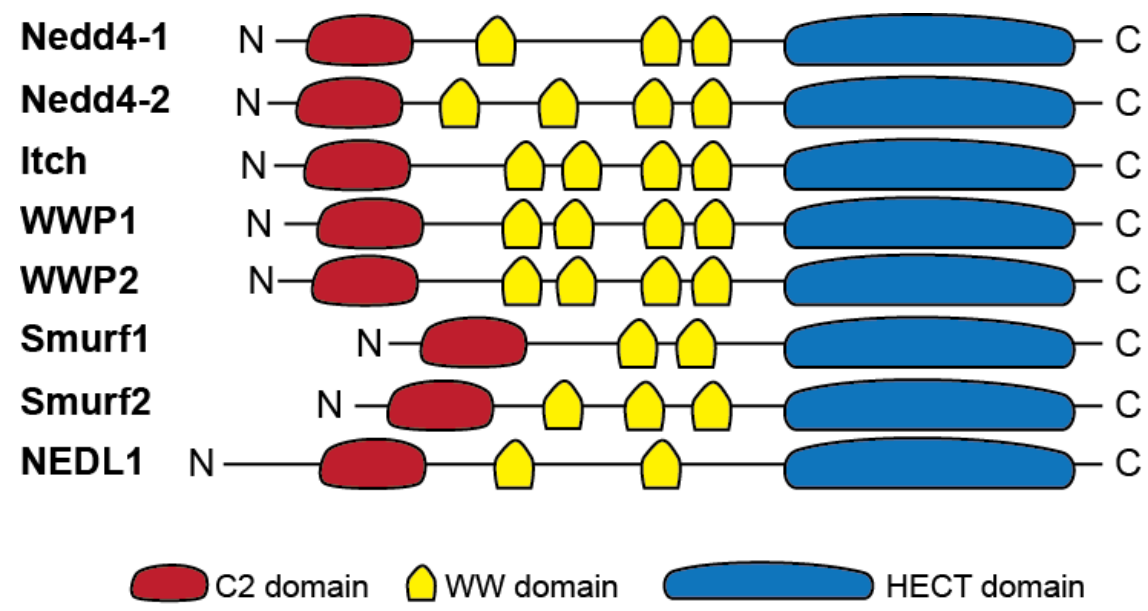

Figure 1-4. Nedd4 Superfamily E3 Ubiquitin Ligases in Mus musculus

There are eight members in the Nedd4 superfamily of the mouse genome. All of them share similar domain structures, including an N-terminal $\mathrm{C} 2$ domain, several WW domains at the central region, and a C-terminal HECT domain. The $\mathrm{C} 2$ domain acts as a $\mathrm{Ca}^{2+}$ sensor to mediate the $\mathrm{Ca}^{2+}$-dependent lipid binding as well as protein-protein interactions. WW domains mediate the interactions with substrate proteins and determine the substrate specificity. The HECT domain is essential for conjugation of ubiquitin onto substrate proteins by forming a thioester bond with an ubiquitin at a conserved cysteine residue, and then transferred the ubiquitin to the substrates.

\subsubsection{Nedd4-1 and Nedd4-2}

Nedd4-1 was initially identified in a screen for developmentally downregulated genes in embryonic mouse brains. Since its discovery, other members of the Nedd4 superfamily were subsequently identified and cloned, including Nedd4-2, the closest isoform of Nedd4-1. Studies on Nedd4-1 and Nedd4-2 deficient mice indicated that Nedd4-1 and Nedd4-2 are essential for several basic cellular functions. Conventional Nedd4-1 KO mice exhibit delayed embryonic development, reduced growth and body weight, and neonatal lethality, likely because of reduced insulin-like growth factor 1 (IGF1)- and insulin- dependent signaling (Cao et al., 2008). Conventional Nedd4$2 \mathrm{KO}$ mice, on the other hand, exhibit increased expression of epithelial sodium channel $(\mathrm{ENaC})$ in lungs, leading to increased airway $\mathrm{Na}^{+}$ reabsorption, depleted airway surface liquid volume, collapsed lungs, and perinatal lethality because of the inability to breath (Boase et al., 2011). 
Regarding their roles in the development of neurons, recent studies based on KO or KD strategies revealed that Nedd4-1 and Nedd4-2 are important for neurite outgrowth (Figure 1-5). Mouse neurons lacking Nedd4-1 show reduced length and complexity of dendrites. The small GTPase Rap2A was identified as the relevant substrate, which is monoubiquitinated or diubiquitinated via a K63-linked chain by Nedd4-1. Ubiquitination of Rap2A by Nedd4-1 blocks the interaction of Rap2A with TNIK, a serine/threonine kinase whose activation by Rap2A leads to reduced dendritic growth. Therefore, the Nedd4-1-mediated Rap2A ubiquitination results in stronger growth and arborization of dendrites in mouse neurons (Kawabe et al., 2010).

In a complementary study, knock down (KD) of Xenopus laevis Nedd4 (xNedd4) was reported to reduce the axonal branching of Xenopus laevis retinal ganglion neurons (Drinjakovic et al., 2010). PTEN was identified as the relevant substrate being polyubiquitinated and targeted to proteosomal degradation by $x$ Nedd4. This notion is supported by a previous report showing that KO of PTEN causes hypertrophy of neurites (Kwon et al., 2006). In addition, experiments employing concomitant KD of both PTEN and xNedd4 rescued the reduced axonal branching phenotype they observed upon xNedd4 KD (Drinjakovic et al., 2010). However, whether PTEN is the physiological substrate of Nedd4-1 remains highly controversial, as an independent report indicated that PTEN is not affected in the Nedd4-1 KO mouse embryonic fibroblasts (Fouladkou et al., 2008). Several other groups also identified alternative E3 ligases for PTEN, including XIAP, WWP2, and CHIP (Ahmed et al., 2012; Maddika et al., 2011; Van Themsche et al., 2009). Notably, xNedd4 is more homologous to mouse Nedd4-2, suggesting the possibility that PTEN is regulated by mouse Nedd4-2, and thus no evidence of PTEN misregulation was obtained in Nedd4-1 KO mouse embryotic fibroblasts (Fouladkou et al., 2008). 


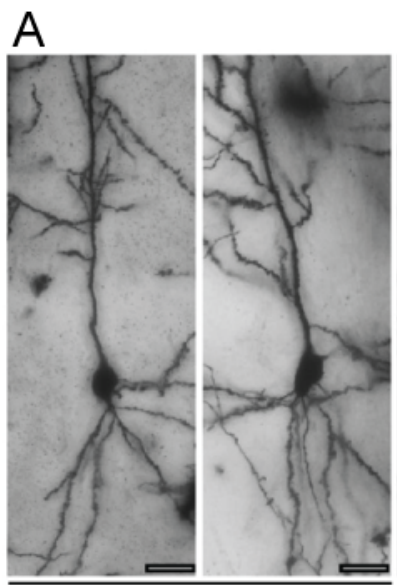

NEX-Cre; Nedd4-1 $f / f$

B

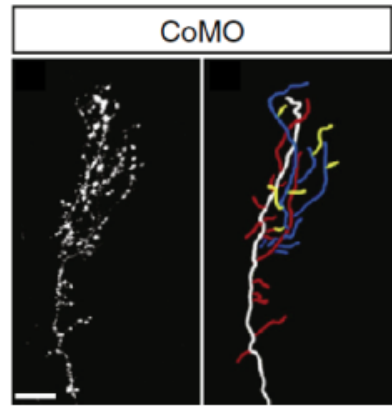

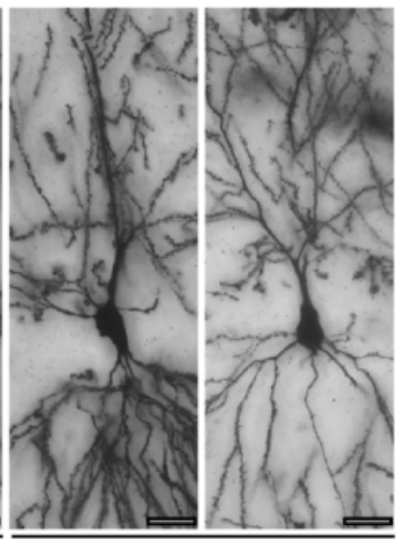

Nedd4-1 $f / f$

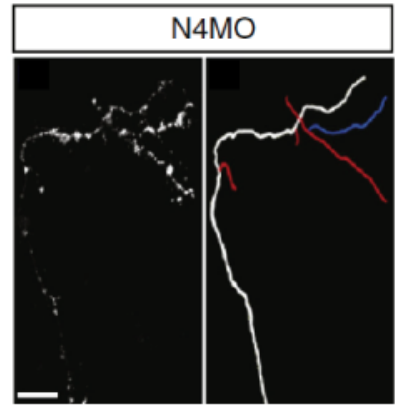

Figure 1-5. Nedd4 Promotes the Growth and Branching of Neurites (A) Hippocampal CA1 neurons from a NEX-Cre;Nedd4-1/f/f mouse (left two panels) show less complex dendritic structures as compared to control neurons from a Nedd4-1 $1^{\mathrm{fff}}$ mouse (right two panels). Scale bars, $20 \mu \mathrm{m}$. Adapted from (Kawabe et al., 2010) with permission from Elsevier, Copyright, 2010. (B) Axon terminals of a Xenopus laevis retinal ganglion neuron shows fewer branches upon KD of XNedd4 (right two panels) as compared to the control (left two panels). Scale bar, $20 \mu \mathrm{m}$. Adapted from (Drinjakovic et al., 2010) with permission from Elsevier, Copyright, 2010.

\subsubsection{WWP1 and WWP2}

WWP1 and WWP2 were originally identified as members of the Nedd4 superfamily based on the presence of tandem WW domains and a domain structure similar to that of Nedd4-1 (Pirozzi et al., 1997). Conventional WWP1 KO mice show increased bone formation as they age, which is accompanied by elevated levels of molecules important for osteoblast differentiation such as JunB, Runx2, and CXCR4 (Shu et al., 2013). WWP1 is often upregulated at both mRNA and protein levels in human breast and 
prostate cancers, indicating an oncogenic role of WWP1. Accordingly, RNAi knockdown of WWP1 suppresses the proliferation of breast and prostate cancer cell lines (Chen et al., 2006, 2009; Li et al., 2009; Nguyen Huu et al., 2008). On the other hand, WWP2 KO mice develop malformations of the craniofacial region, which is associated with monoubiquitination of the transcription factor Goosecoid by WWP2 (Zou et al., 2011). In addition, WWP2 is also implicated in the polyubiquitination and subsequent degradation of PTEN, and therefore plays a role in PI3K-dependent signaling (Maddika et al., 2011). Although multiple studies have indicated roles of WWP1 and WWP2 in general cellular functions, their roles in neuronal development remain mostly unexplored.

Interestingly, in a large-scale RNAi KD screening, the $C$. elegans orthologue of mammalian WWP1 and WWP2 (CeWWP-1) was identified as a candidate gene involved in synaptogenesis at neuromuscular junctions (Sieburth et al., 2005). In a subsequent study, the morphological changes in presynaptic terminals were characterized in loss-of-function mutants of genes that are related to synaptic transmission. CeWWP-1 was found to have significant functional correlations with two presynaptic active zone proteins: sad-1, an orthologue of mammalian sad- $A$ and sad- $B$; and syd-2, an orthologue of mammalian $\alpha$-liprin (Ch'ng et al., 2008). Of note, studies on mouse SAD-A and SAD-B kinases revealed their important roles in the regulation of neuronal polarity formation (Kishi et al., 2005). Together, these studies indicate that mammalian WWP1 and WWP2 may play roles in polarity formation and/or synaptogenesis of neurons.

\subsection{Roles of PTEN in Neuronal Development}

Phosphatase and tensin homolog (PTEN) is one of the most frequently mutated genes found in multiple tumor types. PTEN is a tumor suppressor and a lipid phosphatase that converts phosphatidylinositol-3,4,5trisphosphate $\left(\mathrm{Ptdlns}_{3}\right)$ into phosphatidylinositol-4,5-bisphosphate $\left(P t d I n s P_{2}\right)$ and thereby antagonizes the phosphoinositide-3-kinase- 
dependent (PI3K-dependent) signaling cascades, such as those involved in cell proliferation, cell survival, and protein synthesis (reviewed by Song et al., 2012; Figure 1-6). The prominent functions of PTEN, therefore, make PTEN a prime modulator of numerous cellular processes, including the development of neurons.

In developing mouse brains, the role of PTEN in neurogenesis in vivo was first revealed by a study using Nestin-Cre;PTEN ${ }^{\mathrm{f} / \mathrm{f}}$ mouse line, a neural progenitor specific conditional PTEN KO mouse line. The Nestin-Cre;PTEN/f mice exhibit enlarged brains because of increased cell proliferation and decreased cell death (Groszer et al., 2001). A subsequent study further indicated that PTEN null neural progenitor cells show longer maintenance of the stem cell-like properties, increased G0-G1 cell cycle entry, and decreased growth factor dependency. These data indicate that PTEN operates to suppress the self-renewal capacity of neural progenitor cells (Groszer et al., 2006).

On the other hand, conditional KO of PTEN in postmitotic neurons in mouse brains leads to hypertrophy of neurites and neuronal somata (Chow et al., 2009; Kwon et al., 2001, 2006). Deletion of PTEN in neurons also results in increased density of spines, many of which show abnormal morphology such as lack of the distinct mushroom-shaped termini, enlarged presynaptic terminals packed with increased numbers of synaptic vesicles but no obvious corresponding postsynaptic density, and subsequent aberrant synaptic transmission (Fraser et al., 2008; Luikart et al., 2011). Moreover, KD of PTEN in cultured neurons results in multiple axons projecting from a single neuron, which is caused by abnormal inhibition of the GSK3 $\beta$ activity (Jiang et al., 2005). In short, these reports indicate that PTEN plays critical roles in multiple stages of neuronal development, including regulation of neuronal proliferation, neurite outgrowth, synapse development, and neuronal polarity formation. 


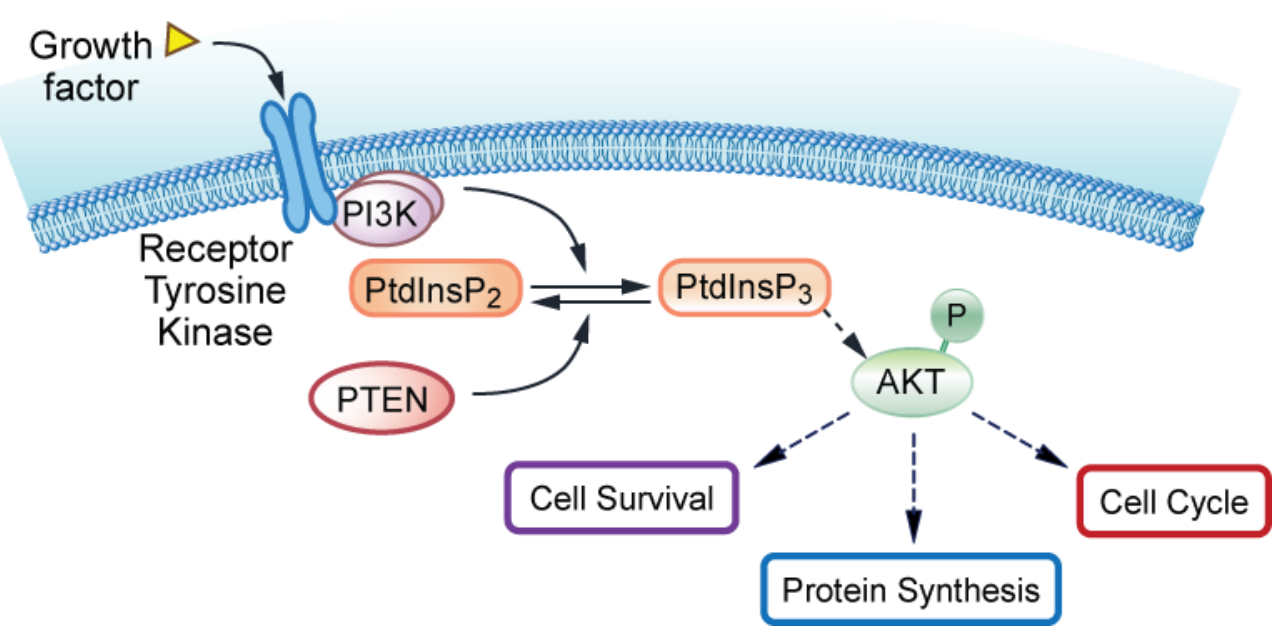

Figure 1-6. Major Cellular Functions of the PI3K/PTEN-Dependent Signaling Pathways

The PI3K/PTEN-dependent signaling plays key roles in regulating multiple cellular functions. PI3K catalyzes the phosphorylation of $\mathrm{PtdlnsP}_{2}$ to generate PtdlnsP $\mathrm{P}_{3}$ in response to stimulations (e.g. growth factors). PTEN antagonizes the effect of $\mathrm{PI} 3 \mathrm{~K}$ by converting Ptdlns $\mathrm{P}_{3}$ back to $\mathrm{PtdlnP}_{2}$. Elevated PtdlnsP 3 levels lead to the phosphorylation of AKT, which further regulates signaling cascades such as those involved in cell survival, protein synthesis, and cell cycle progression.

\subsection{Roles of Cdk5 in Neuronal Development}

Cyclin-dependent kinase 5 (Cdk5) was initially identified based on its close sequence homology to the human cdc2 kinase and was therefore considered as a member of the serine/threonine cyclin-dependent kinase (Cdk) family (Lew et al., 1992; Meyerson et al., 1992). Unlike other members in the Cdk family, Cdk5 does not directly act in cell cycle regulation. In addition, the activation of Cdk5 does not depend on the binding to cyclin, but rather on binding to its specific activators, p35 and p39. Although Cdk5 is ubiquitously expressed in all tissues, its highest expression and corresponding kinase activity are detected in postmitotic neurons because of the neuron-specific expression patterns of p35 and p39 (Tang et al., 1995; Tsai et al., 1993, 1994). Since the cloning of Cdk5, numerous studies have discovered critical roles of Cdk5 in the nervous system, including the development of neurons (Figure 1-7; reviewed by Dhavan and Tsai, 2001; Su and Tsai, 2011). 
Cdk5 KO mice die perinatally and show severe neocortical lamination deficits, as the newborn neurons in Cdk5 KO brains are unable to migrate through the earlier-generated neurons (Ohshima et al., 1996, 2007). Interestingly, these defects found in Cdk5 KO mice are recapitulated in p35 $\mathrm{KO}$ and $p 35 ; p 39$ double KO mice. Notably, $p 35 \mathrm{KO}$ mice are viable and show a milder phenotype as compared to Cdk5 KO and p35;p39 double KO mice. (Chae et al., 1997; Ko et al., 2001). These studies on the respective KO animals indicate that activation of Cdk5 by p35 or p39 is essential for proper neuronal migration and neocortical lamination during embryonic cortical development. Several key substrates have been reported as phosphorylation targets of Cdk5 in neuronal migration, such as NUDEL, a Lis1-interating protein whose intracellular distribution and interaction with dynein are affected by Cdk5-mediated phosphorylation (Niethammer et al., 2000; Sasaki et al., 2000); the tyrosine kinase FAK, which is phosphorylated by Cdk5 to promote the microtubule organization for nucleokinesis during neuronal migration (Xie et al., 2003); and doublecortin (Dcx), whose phosphorylation by Cdk5 decreases the affinity to microtubules and thus increases microtubule dynamics to allow for neuronal migration (Tanaka et al., 2004).

In addition to the roles of Cdk5 in neuronal migration, Cdk5 also plays key roles in the regulation of neurite outgrowth and axonal pathfinding during neuronal development. Overexpression of a dominant negative Cdk5 variant (Cdk5 D144N) in cultured neurons causes a reduction in neurite outgrowth, whereas Cdk5/p35 overexpression leads to longer neurites (Nikolic et al., 1996; Paglini et al., 1998). One of the identified substrates in this regard is p21-activated kinase 1 (Pak1), whose kinase activity is inhibited upon phosphorylation by Cdk5, and its inhibition promotes actin dynamics to allow for neurite outgrowth (Nikolic et al., 1996). Further studies have also indicated the importance of Cdk5-mediated phosphorylation of receptor tyrosine kinase, TrkB, for BDNF-stimulated neurite outgrowth (Cheung et al., 2007), and phosphorylation of collapsing response mediator protein-2 (CRMP-2) for semaphorin 3A-induced growth cone collapse (Brown et al., 2004). 
Cdk5 is localized to neuronal synapses (Humbert et al., 2000). Acute Cdk5 gain-of-function in vivo also results in a dramatic increase in synapse numbers (Fischer et al., 2005). Correspondingly, the membrane associated guanylate kinase family member CASK was identified as a presynaptic substrate of Cdk5. Phosphorylation of CASK by Cdk5 recruits CASK to presynaptic terminals, where it interacts with several presynaptic proteins, including Neurexin, Veli, and Mint1, and thereby promotes synapse formation (Samuels et al., 2007). On the other hand, the postsynaptic role of Cdk5 during neuronal development was revealed by a study showing that the Cdk5-dependent phosphorylation of the N-terminal domain of PSD-95 is important for regulating the clustering of PSD-95, as well as some of the postsynaptic receptors such as NMDA receptors (Morabito et al., 2004). Together, these results indicate a role of Cdk5 in the formation and/or maintenance of synapses.

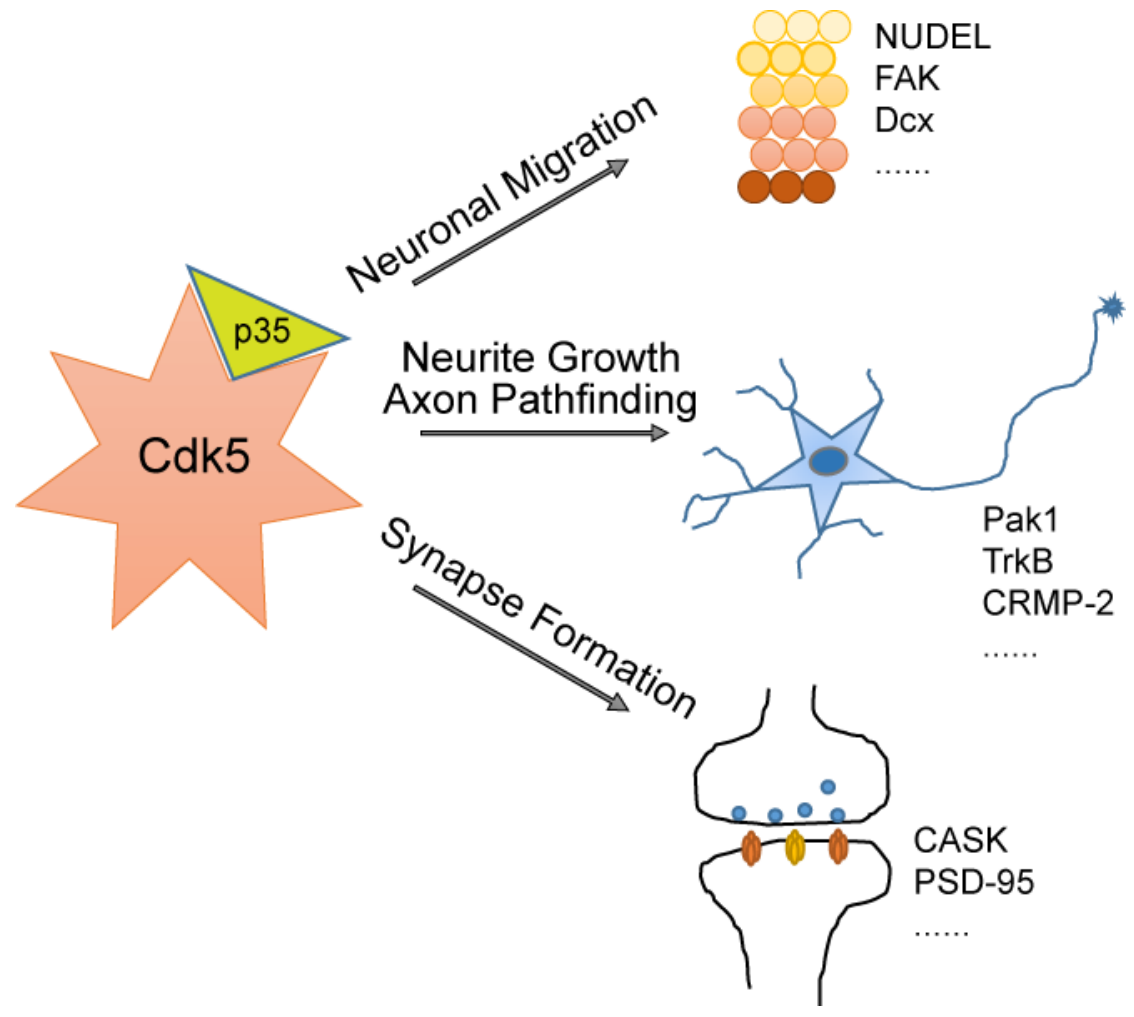

Figure 1-7. Roles of Cdk5 in the Development of Mammalian Neurons Cdk5 activity is crucial for multiple cellular processes during mammalian neuronal development. The activation of Cdk5 requires the binding to the Cdk5 activator, p35. Upon activation, Cdk5 regulates various neuronal developmental processes such as neuronal migration, neurite outgrowth, 
axon pathfinding, and synapse formation. Multiple substrate proteins that are phosphorylated by Cdk5 have been identified to be relevant for the regulation of these processes. Dcx, doublecortin; NUDEL, nudE nuclear distribution gene $\mathrm{E}$ homolog-like 1; FAK, focal adhesion kinase; Pak1, p21-activated kinase 1; TrkB, neurotrophic tyrosine kinase receptor type 2; CRMP-2, collapsing response mediator protein-2; PSD-95, postsynaptic density protein-95.

\subsection{Aims of the Present Study}

The present study was designed to unveil the roles of several ubiquitin E3 ligases of the Nedd4 superfamily (Nedd4-1, Nedd4-2, WWP1, and WWP2) in rodent brain development, and to characterize possible interplays between ubiquitination and other signaling pathways such as protein phosphorylation during neuronal development.

In an attempt to resolve the long-lasting dispute on whether PTEN is a physiological substrate of Nedd4-1 and Nedd4-2, I focused in the first part of this study on clarifying the relationship between PTEN and the Nedd4 subfamily E3 ligases, Nedd4-1 and Nedd4-2, in neurite development. In the second part of this study, I focused on characterizing the roles of the WWP subfamily E3 ligases, WWP1 and WWP2, in the developing mouse brain by identifying their binding partners and ubiquitination substrates. 


\section{Materials and Methods}

\subsection{Animals}

The Nedd4-1 ${ }^{f / f}$ (Kawabe et al., 2010), Nedd4-2/f/f (Kimura et al., 2011), $W W P 1^{f / f}$, and $W W P 2^{f / f}$ mouse lines were generated by Dr. Hiroshi Kawabe and Prof. Dr. Nils Brose. The NEX-Cre mouse line was generated and provided by Dr. Goebbles and Prof. Dr. Klaus A. Nave (Goebbels et al., 2006). The $P T E N^{f / f}$ mouse line was generated and provided by Dr. Hong Wu (Groszer et al., 2001). All animal experiments were approved by the responsible local government (Landesamtes für Verbraucherschutz und Lebensmittelsicherheit Niedersachsen) and conducted in compliance with German guidelines [comparable to National Institutes of Health (NIH) guidelines].

\subsection{Reagents}

\subsubsection{Chemicals}

\begin{tabular}{|l|l|}
\hline Acrylamide/N,N'-Methylene-bis-Acrylamide & National Diagnositic \\
\hline Adenosine Triphosphate (ATP) & Sigma-Aldrich \\
\hline Agarose (UltraPure agarose) & Invitrogen \\
\hline Ammonium Persulfate (APS) & Sigma-Aldrich \\
\hline Ampicillin & Invitrogen \\
\hline Aprotinin & Roche \\
\hline Bacto-Agar & DIFCO, BD \\
\hline Bacto-Casamino Acids & DIFCO, BD \\
\hline Bacto-Peptone & DIFCO, BD \\
\hline Bacto-Yeast Extract & DIFCO, BD \\
\hline Boric Acid & Sigma-Aldrich \\
\hline Bovine serum albumin (BSA), Fraction V & Pierce, Thermo \\
\hline CHAPS & Biomol Feinchemikalien \\
\hline Comassie Brilliant Blue R250 & BioMol Feinchemikalien \\
\hline Cycloheximide & Sigma-Aldrich \\
\hline Darbecco Modified Eagle's Medium (D'MEM) & Gibco, Invitrogen \\
\hline Dimethyl Sulfoxide (DMSO) & Sigma-Aldrich \\
\hline Dithiothreitol (DTT) & Sigma-Aldrich \\
\hline DNA Ladder Mix Sample, GeneRuler & Fermentas \\
\hline dNTPs & GE Healthcare \\
\hline
\end{tabular}




\begin{tabular}{|c|c|}
\hline Skim Milk & Nestle \\
\hline ECL Reagent & Amersham, GE Healthcare \\
\hline Ethanol & Sigma-Aldrich \\
\hline Ethidium Bromide (1\% solution) & Carl Roth \\
\hline Ethylene Glycol Tetraacetic Acid (EGTA) & Sigma-Aldrich \\
\hline Ethylenediaminetetraacetic Acid (EDTA) & Sigma-Aldrich \\
\hline FastGreen & Sigma-Aldrich \\
\hline GelRed & Biotium \\
\hline Glucose & Sigma-Aldrich \\
\hline Glutathione (GSH) & Sigma-Aldrich \\
\hline Glycerol & Sigma-Aldrich \\
\hline Glycine & Sigma-Aldrich \\
\hline Goat Serum & Gibco, Invitrogen \\
\hline HEPES & Sigma-Aldrich \\
\hline Hydrochloric Acid $(\mathrm{HCl})$ & Sigma-Aldrich \\
\hline lodoacetamide & Sigma-Aldrich \\
\hline IPTG & BioMol Feinchemikalien \\
\hline Kanamycin & Invitrogen \\
\hline Leupeptin & Roche \\
\hline Luria Broth (LB) & Sigma-Aldrich \\
\hline Magnesium Chloride $\left(\mathrm{MgCl}_{2} 6 \mathrm{H}_{2} \mathrm{O}\right)$ & Sigma-Aldrich \\
\hline Methanol & Sigma-Aldrich \\
\hline $\mathrm{N}$-Ethylmaleimide $\left(\mathrm{C}_{6} \mathrm{H}_{7} \mathrm{NO}_{2}, \mathrm{NEM}\right)$ & Sigma-Aldrich \\
\hline Neurobasal A Medium & Gibco, Invitrogen \\
\hline $\begin{array}{l}N^{\prime} N^{\prime} N^{\prime}-\text { Tetramethylethyl Enediamine } \\
\text { (TEMED) }\end{array}$ & BioRad \\
\hline Opti-MEM I & Invitrogen \\
\hline Phosphate Buffered Saline (PBS) & PAA Laboratories \\
\hline Phenylmethylsulfonyl Fluoride (PMSF) & Roche \\
\hline Protein Molecular Weight Standards & Invitrogen \\
\hline Rapamycin & Cell Signaling \\
\hline Restriction Endonucleases & $\begin{array}{l}\text { New England Biolabs } \\
\text { (NEB) }\end{array}$ \\
\hline RNase OUT & Invitrogen \\
\hline Sodium Butyrate & Merck \\
\hline Sodium Chloride & Sigma-Aldrich \\
\hline Sodium Deoxycholate & Sigma-Aldrich \\
\hline Sodium Dodecyl Sulfate (SDS) & Roche \\
\hline Sodium phosphate $\left(\mathrm{NaH}_{2} \mathrm{PO}_{4}\right)$ & Roche \\
\hline Sucrose & Sigma-Aldrich \\
\hline Triton X-100 & Roche \\
\hline Tween 20 & Sigma-Aldrich \\
\hline Ubiquitin & Boston Biochem \\
\hline X-Gal & BioMol \\
\hline Z-Leu-Leu-Leu-CHO (MG-132) & Boston Biochem \\
\hline
\end{tabular}




\subsubsection{Enzymes from Commercial Sources}

\begin{tabular}{|l|l|}
\hline Gateway LR Clonase Enzyme Mix & Invitrogen \\
\hline Alkaline Phosphatase & Roche \\
\hline DNasel (RNase free) & Roche \\
\hline Pfu Polymerase & Stratagene \\
\hline SuperScript III RNase H reverse transcriptase & Invitrogen \\
\hline SYBR Green PCR master mix & Applied Biosystems \\
\hline T4 DNA Ligase & Invitrogen \\
\hline Taq Polymerase (REDTaq) & Sigma-Aldrich, D4309 \\
\hline Ubiquitin Activating Enzyme (UBE1) & Boston Biochem \\
\hline UbcH5b & Boston Biochem \\
\hline UbcH6 & Boston Biochem \\
\hline UbcH7 & Boston Biochem \\
\hline
\end{tabular}

\subsubsection{Kits}

\begin{tabular}{|l|l|}
\hline PureLink Quick Plasmid Miniprep Kit & Invitrogen \\
\hline PureLink HiPure Plasmid Midiprep Kit & Invitrogen \\
\hline PureLink HiPure Plasmid Maxiprep Kit & Invitrogen \\
\hline EndoFree Plasmid Maxi Kit & QIAGEN \\
\hline PureLink Gel Extraction Kit & Invitrogen \\
\hline QuickChange II Site-Directed Mutagenesis Kit & Stratagene \\
\hline TOPO TA Cloning Kit & Invitrogen \\
\hline PTEN Phosphatase Activity Kit & Echelon \\
\hline NucleoSpin RNA XS Kit & $\begin{array}{l}\text { Macherey- } \\
\text { Nagel }\end{array}$ \\
\hline BCA Protein Assay Kit & Thermo, Pierce \\
\hline
\end{tabular}

\subsubsection{Bacterial Strains}

\begin{tabular}{|l|l|}
\hline E. coli XL-1 Blue competent cells & Stratagene \\
\hline E. coli Electro10-Blue competent cells & Stratagene \\
\hline E. coli JM109 competent cells & Promega \\
\hline E. coli TOP10 competent cells & Invitrogen \\
\hline E.coli ccdB survival competent cells & Invitrogen \\
\hline E. coli BL21 Rosetta competent cells & Stratagene \\
\hline
\end{tabular}

\subsubsection{Vector Plasmids}

\begin{tabular}{|l|l|}
\hline pCRII TOPO & Invitrogen \\
\hline pCRII TOPO-WWP2 WT & Provided by Dr. Mika-Kishimoto Suga \\
\hline pCRII TOPO-Myc-Cdk5 WT & Generated in this study \\
\hline
\end{tabular}




\begin{tabular}{|l|l|}
\hline pCIG-Flag-Nuak1 & Provided by Dr. Franck Polleux \\
\hline pCIG-HA-Nuak2 & Provided by Dr. Franck Polleux \\
\hline pCX-myrVenus & $\begin{array}{l}\text { Provided by Dr. Anna-Katerina } \\
\text { Hadjantonakis }\end{array}$ \\
\hline pCIneoHA & Provided by Dr. Hiroshi Kawabe \\
\hline pCMVdeltaR8.2 & Provided by Prof. Dr. Pavel Osten \\
\hline pCIneoHA-p25 & Generated in this study \\
\hline pClneoMyc & Provided by Dr. Hiroshi Kawabe \\
\hline pCIneoMyc-Cdk5 WT & Generated in this study \\
\hline pCIneoMyc-WWP1 WT & Provided by Dr. Mika-Kishimoto Suga \\
\hline pcDNA3.1/nV5-DEST & Invitrogen \\
\hline pcDNA3.1/nMyc-DEST & Generated in this study \\
\hline pcDNA3.1/nMyc-Tubulin4A & Generated in this study \\
\hline pcDNA3.1/nMyc-Uba1 & Generated in this study \\
\hline pcDNA3.1/nMyc-Prkar1b & Generated in this study \\
\hline pcDNA3.1/nMyc-Map3k6 & Generated in this study \\
\hline pDONR221-Uba1 & DNASU \#HsCD00076423 \\
\hline pDONR221-Prkar1b & DNASU \#HsCD00296901 \\
\hline pEF1-V5-Usp9x & Provided by Dr. Hiroshi Kawabe \\
\hline pENTR223-Map3k6 & DNASU \#HsCD00505767 \\
\hline pENTR223-TUBA4A & DNASU \#HsCD00511991 \\
\hline pFUGW & Salk Inst., Dr.Inder M. Verma \\
\hline pFUGW-iCre & Provided by Dr. Richard L. Huganir \\
\hline pFUGW-WWP1 WT & Generated in this study \\
\hline pFUGW-WWP2 WT & Generated in this study \\
\hline pGEX4T-1 & GE Healthcare Life Sciences \\
\hline pGEX4T-1 WWP1 WT & Provided by Dr. Hiroshi Kawabe \\
\hline pGEX4T-1 WWP1 C886S & Generated in this study \\
\hline pGEX6P-1 & GE Healthcare Life Sciences \\
\hline pGEX6P-1-Cdk5 WT & Generated in this study \\
\hline pGEX6P-1-Cdk5 P234A, \\
Y236F & Generated in this study \\
\hline pGEX6P-1-WWP2 WT & Provided by Dr. Hiroshi Kawabe \\
\hline pGEX6P-2-p35 & Addgene \#24895 \\
\hline pRRLsinPPT-CMV-WPRE & Provided by Dr. Luigi Naldini \\
\hline $\begin{array}{l}\text { pRRLsinPPT-CMV-WPRE- } \\
\text { Myc-Cdk5 WT }\end{array}$ & Generated in this study \\
\hline pMalC2 & New England Biolabs \\
\hline pMalC2-p25 & Generated in this study \\
\hline pMD2.G & Provided by Prof. Dr. Pavel Osten \\
\hline pRaichuMyc & Provided by Dr. Hiroshi Kawabe \\
\hline pRaichuMyc-Cdk5 WT & Provided by Dr. Hiroshi Kawabe \\
\hline pRaichuMyc-Cdk5 K61R & Generated in this study \\
\hline pRK5-HA-Ubiquitin & $\begin{array}{l}\text { Provided by Dr. Hans-Jürgen } \\
\text { Kreienkamp }\end{array}$ \\
\hline
\end{tabular}




\subsubsection{Oligonucleotides}

Oligonucleotide primers used in the present study are listed below. They were synthesized in the Max-Planck-Institute for Experimental Medicine DNA Core Facility on an ABI 5000 DNA/RNA synthesizer. Restriction sites used for molecular cloning are underlined when applicable.

\begin{tabular}{|c|c|c|}
\hline Primer \# & Sequence (5'-3') & $\begin{array}{l}\text { Rest. } \\
\text { site }\end{array}$ \\
\hline 28606 & AACGAATTCGCCCAGCCCCCACCGG & EcoRI \\
\hline 28607 & TTCCGCTCGAGTCACCGATCCAGGCCTAGG & Xhol \\
\hline 30937 & $\begin{array}{l}\text { GCGCCAAGCTTACCATGGAACAGAAACTGATCT } \\
\text { CTGAAGAAGACCTGGAT }\end{array}$ & HindIII \\
\hline 30938 & $\begin{array}{l}\text { ATCCAGGTCTTCTTCAGAGATCAGTTTCTGTTCC } \\
\text { ATGGTAAGCTTGGCGC }\end{array}$ & HindIII \\
\hline 31918 & $\begin{array}{l}\text { ACCAAGCTGGCAGACTTTAAGCCCTACCCAATG } \\
\text { TACCC }\end{array}$ & \\
\hline 31919 & $\begin{array}{l}\text { GTAGGGCTTAAAGTCTGCCAGCTTGGTCATGGC } \\
\text { AGGCC }\end{array}$ & \\
\hline 27283 & GTTCTCGAGCTATGGGGGACAGAAGTCAG & Xhol \\
\hline 26356 & $\begin{array}{l}\text { AGCATGAACCACCAGGTCA } \\
\text { (RT-qPCR Nedd4-1 forward) }\end{array}$ & \\
\hline 26357 & $\begin{array}{l}\text { TTTTTCCGAATCCATCATCC } \\
\text { (RT-qPCR Nedd4-1 reverse) }\end{array}$ & \\
\hline 26358 & $\begin{array}{l}\text { AATGACCTGGGCCCTCTT } \\
\text { (RT-qPCR Nedd4-2 forward) }\end{array}$ & \\
\hline 26359 & $\begin{array}{l}\text { GTAAAACGTGCGGCCATC } \\
\text { (RT-qPCR Nedd4-2 reverse) }\end{array}$ & \\
\hline 26360 & $\begin{array}{l}\text { CATTGAGATTGCCGCTTACA } \\
\text { (RT-qPCR Neurofilament } H \text { forward) }\end{array}$ & \\
\hline 26361 & $\begin{array}{l}\text { ACTCGGACCAAAGCCAATC } \\
\text { (RT-qPCR Neurofilament } H \text { reverse) }\end{array}$ & \\
\hline 19900 & ATCССТCCACCCTATGACAA & \\
\hline 19901 & GCCCCAGGTAAGCAAACTT & \\
\hline 11282 & GGATCTGCTGGCCCCATAC & \\
\hline 11283 & CTTTCCAACGCCAGCACCT & \\
\hline - & $\begin{array}{l}\text { GGCTCATGACCACAGTCCA } \\
\text { (RT-qPCR GAPDH forward) }\end{array}$ & \\
\hline
\end{tabular}




\begin{tabular}{|c|l|c|}
\hline- & $\begin{array}{l}\text { TCCACAGTCTTCTGGGTGG } \\
\text { (RT-qPCR GAPDH reverve) }\end{array}$ & \\
\hline 30007 & $\begin{array}{l}\text { ACAGGATCCACCATGGAGCAGAAGCTTATCAGC } \\
\text { G }\end{array}$ & BamHI \\
\hline
\end{tabular}

\subsubsection{Antibodies}

Primary antibodies used in the present study

\begin{tabular}{|l|l|l|l|l|l|}
\hline \multirow{2}{*}{ Antibody } & \multirow{2}{*}{$\begin{array}{l}\text { Host } \\
\text { Species }\end{array}$} & Origin & \multicolumn{4}{|l|}{ Usage and Dilution } \\
\cline { 4 - 6 } Actin (AC40) & Mouse & $\begin{array}{l}\text { Sigma- } \\
\text { Aldrich }\end{array}$ & $1: 500$ & - & - \\
\hline Actin & Rabbit & $\begin{array}{l}\text { Sigma- } \\
\text { Aldrich }\end{array}$ & $1: 2000$ & - & - \\
\hline AKT & Rabbit & $\begin{array}{l}\text { Cell } \\
\text { Signaling }\end{array}$ & $1: 2000$ & - & - \\
\hline $\begin{array}{l}\text { phospho-AKT } \\
\text { (S473) }\end{array}$ & Rabbit & $\begin{array}{l}\text { Cell } \\
\text { Signaling }\end{array}$ & $1: 2000$ & - & - \\
\hline Ankyrin G & Rabbit & Santa Cruz & - & $1: 400$ & $1: 400$ \\
\hline Cdk5 & Rabbit & Santa Cruz & $1: 200$ & - & - \\
\hline Cdk5 (DC19) & Mouse & Millipore & $1: 200$ & - & - \\
\hline Cre & Mouse & Sigma & $1: 200$ & $1: 200$ & - \\
\hline Cux1 & Rabbit & Santa Cruz & - & - & $1: 200$ \\
\hline FLAG (M2) & Mouse & Sigma & $1: 1000$ & - & $1: 1000$ \\
\hline GFP (7.1/13.1) & Mouse & Roche & $1: 1000$ & - & $1: 1000$ \\
\hline GFP & Chicken & Aves Lab & - & $1: 2000$ & - \\
\hline GSK3 $\beta$ & Mouse & $\begin{array}{l}\text { BD } \\
\text { Biosciences }\end{array}$ & $1: 2000$ & - & - \\
\hline HA (HA.11) & Mouse & Covance & $1: 1000$ & - & - \\
\hline Nedd4-1 & Mouse & $\begin{array}{l}\text { BD } \\
\text { Biosciences }\end{array}$ & $1: 500$ & - & - \\
\hline Nedd4-2 & Rabbit & $\begin{array}{l}\text { Cell } \\
\text { Signaling }\end{array}$ & $1: 2000$ & - & - \\
\hline NeuN (A60) & Mouse & Millipore & - & - & $1: 100$ \\
\hline MAP2 & Rabbit & Millipore & - & - & $1: 2000$ \\
\hline MAP2 & Chicken & Novus & - & $1: 2000$ & $1: 2000$ \\
\hline MBP & Mouse & $\begin{array}{l}\text { Cell } \\
\text { Signaling }\end{array}$ & $1: 5000$ & - & - \\
\hline C-Myc (9E10) & Mouse & Sigma & $1: 1000$ & - & - \\
\hline C-Myc & Rabbit & Santa Cruz & $1: 1000$ & - & $1: 500$ \\
\hline PTEN & Rabbit & $\begin{array}{l}\text { Cell } \\
\text { Signaling }\end{array}$ & $1: 500$ & $1: 250$ & - \\
\hline PTEN (6H2.1) & Mouse & Millipore & $1: 500$ & - & - \\
\hline$\beta-T u b u l i n(T U B 2.1)$ & Mouse & Sigma & $1: 5000$ & - & - \\
\hline phospho-S6 & Rabbit & Cell & $1: 2000$ & - & - \\
\hline & & & & & \\
\hline
\end{tabular}




\begin{tabular}{|l|l|l|l|l|l|}
\hline (Ser235/236) & & Signaling & & & \\
\hline Ubiquitin & Rabbit & Dako & $1: 500$ & - & - \\
\hline Ubiquitin (P4D1) & Mouse & Santa Cruz & $1: 500$ & - & - \\
\hline V5 & Mouse & $\begin{array}{l}\text { Cell } \\
\text { Signaling }\end{array}$ & $1: 500$ & - & - \\
\hline WWP1 (0221) & Rabbit & SySy & $1: 200$ & - & - \\
\hline WWP2 (0217) & Rabbit & SySy & $1: 200$ & - & - \\
\hline
\end{tabular}

Secondary antibodies used in the present study.

\begin{tabular}{|l|l|l|l|l|}
\hline & $\begin{array}{l}\text { Host } \\
\text { Species }\end{array}$ & $\begin{array}{l}\text { Conjugated } \\
\text { substrate/Dye }\end{array}$ & Origin & $\begin{array}{l}\text { Usage, } \\
\text { Dilution }\end{array}$ \\
\hline a-Mouse IgG & Goat & HRP & BioRad & $\begin{array}{l}\text { WB, } \\
1: 20000\end{array}$ \\
\hline a-Rabbit IgG & Goat & HRP & BioRad & $\begin{array}{l}\text { WB, } \\
1: 20000\end{array}$ \\
\hline a-Mouse IgG & Goat & IL-COR IRDye 800 & Rockland & WB, 1:2000 \\
\hline a-Rabbit IgG & Goat & IL-COR IRDye 680 & Rockland & WB, 1:2000 \\
\hline a-Chicken IgG & Goat & $\begin{array}{l}\text { Alexa Fluor } \\
\text { 488/633 }\end{array}$ & Invitrogen & $\begin{array}{l}\text { IHC/ICC, } \\
1: 1000\end{array}$ \\
\hline a-Mouse IgG & Goat & $\begin{array}{l}\text { Cy5/ Alexa Fluor } \\
350 / 488 / 555\end{array}$ & Invitrogen & $\begin{array}{l}\text { IHC/ICC, } \\
1: 1000\end{array}$ \\
\hline a-Rabbit IgG & \multirow{2}{*}{ Goat } & $\begin{array}{l}\text { Alexa Fluor } \\
350 / 488 / 555 / 633\end{array}$ & Invitrogen & $\begin{array}{l}\text { IHC/ICC, } \\
1: 1000\end{array}$ \\
\hline
\end{tabular}

\subsection{Molecular Biology}

\subsubsection{Bacteria Transformation}

An aliquot $(50 \mu \mathrm{l})$ of electro-competent $E$. coli cells was thawed on ice and transferred to a pre-cooled electroporation cuvette (0.1 cm, BioRad). $1 \mu \mathrm{l}$ of DNA sample was added to the $E$. coli cells and mixed gently. The cuvette was then applied for an electric pulse of $1.80 \mathrm{kV}(E$. coli pulser, BioRad). Immediately following the electroporation, $E$. coli cells were retrieved from the cuvette with $1 \mathrm{ml} \mathrm{LB}$ medium and allowed for recovery for $1 \mathrm{~h}$ at $37^{\circ} \mathrm{C}$ under moderate shaking. $E$. coli cells were then centrifuged at $10,000 \mathrm{~g}$ for 1 $\mathrm{min}$, and the pellet was resuspended in $50 \mu \mathrm{L}$ LB medium and plated on the appropriate selection plates. 
LB medium:

$25 \mathrm{~g}$ Lutia Broth (LB; Invitrogen) powder was dissolved in $1 \mathrm{~L}$ distilled $\mathrm{H}_{2} \mathrm{O}$ $\left(\mathrm{ddH}_{2} \mathrm{O}\right)$ and autoclaved.

LB plates:

$15 \mathrm{~g}$ Bacto-agar (Invitrogen) was added in $1 \mathrm{~L}$ of LB medium and autoclaved. The required selection antibiotic was added after autoclaving.

\subsubsection{Plasmid DNA preparation}

The plasmid DNA preparation was carried out using the PureLink Quick Plasmid Miniprep Kit, HiPure Plasmid Midiprep Kit, HiPure Plasmid Maxiprep Kit (Invitrogen), or EndoFree Plasmid Maxi Kit (QIAGEN) according to the manufacturers' instructions and resuspended in TE buffer.

TE buffer:

$10 \mathrm{mM}$ Tris- $\mathrm{HCl} \mathrm{pH}$ 7.4, $1 \mathrm{mM}$ EDTA

\subsubsection{Sequencing of DNA}

All DNA sequence analysis was done in the MPI-EM DNA Core Facility on an Applied Biosystems 373 DNA Sequencer.

\subsubsection{Gateway Cloning}

Gateway LR reactions were carried out using the Gateway LR Clonase Enzyme Mix (Invitrogen). A reaction mix containing $1 \mu$ l entry clone plasmid (100-300 $\mathrm{ng} / \mu \mathrm{l}), 1 \mu \mathrm{l}$ destination vector (150 $\mathrm{ng} / \mu \mathrm{l}), 0.5 \mu \mathrm{l}$ reaction buffer, and $0.5 \mu \mathrm{l} \mathrm{LR}$ Clonase was prepared and incubated at $25^{\circ} \mathrm{C}$ overnight. $1 \mu \mathrm{l}$ of the reaction mix was then transformed into $E$. coli TOP10 competent cells and plated on the appropriate antibiotic selection plates. Plasmid DNA from the positive E. coli colonies was isolated and subjected to sequencing to confirm the sequence. 


\subsubsection{DNA Digestion with Restriction Endonucleases}

The instruction manuals provided by New England BioLabs were referred to for all DNA digestion procedures. Generally, the appropriate quantity of DNA was digested for 1-3 $\mathrm{h}$ at the enzyme specific temperature in the appropriate buffer.

\subsubsection{Agarose Gel Electrophoresis}

For size analyses and purification of DNA, DNA products were subjected to agarose gel electrophoresis. Generally, a TBE based-agarose gel $(0.7-2 \%)$ containing ethidium bromide or GelRed was used. Negatively charged DNA was separated at a constant current (100-140 mA) in TBE buffer and visualized by ethidium bromide or GelRed under UV light exposure. GeneRuler DNA Ladder Mix sample (Fermentas) was loaded in parallel as the marker.

TBE buffer:

$50 \mathrm{mM}$ Tris-Base, $50 \mathrm{mM}$ boric acid, 2 mM EDTA, pH 8.0

\subsubsection{Purification of DNA Fragments}

Following the separation by agarose gel electrophoresis, DNA fragments of interest were excised and isolated from agarose gels using the PureLink Gel Extraction Kit (Invitrogen) according to the manufacturer's protocol.

\subsubsection{De-phosphorylation of 5'-DNA Ends}

Dephosphorylation of the 5'-ends of DNA plasmids with compatible ends was carried out in order to prevent the self-ligation of vectors in the further DNA ligation procedures. Plasmid DNA was treated with alkaline phosphatase (Roche) in the supplied buffer for $15 \mathrm{~min}$ at $37^{\circ} \mathrm{C}$, according to 
manufacturer's instructions. The alkaline phosphatase was then inactivated by incubation at $65^{\circ} \mathrm{C}$ for $15 \mathrm{~min}$.

\subsubsection{DNA Ligation}

Plasmid and insert DNA with compatible ends were mixed in a molar ratio of $1: 1$ to $1: 10$, supplemented with additional $2 \mathrm{mM}$ ATP, T4 DNA ligase (Invitrogen), and the ligase-specific buffer in $20 \mu \mathrm{l}$ of reaction volume. The ligation reaction was taken place at $25^{\circ} \mathrm{C}$ for $1.5 \mathrm{~h}$, or at $16^{\circ} \mathrm{C}$ for $16-20 \mathrm{~h}$.

\subsubsection{Polymerase Chain Reaction (PCR)}

DNA fragments of interest were amplified in 20-25 $\mu$ l of reaction mixtures containing the double stranded DNA template, oligonucleotide primers, dNTPs, and pfu DNA polymerase (Stratagene) in the appropriate reaction buffer. PCR reactions were run on a Gene Amp 9700 PCR cycler (Applied Biosystems) with basic cycle parameters as below:

Step 1: $94^{\circ} \mathrm{C}$ for 2 min

Step 2: $94^{\circ} \mathrm{C}$ for $20 \mathrm{~s}$

Step 3: annealing temperature for $20 \mathrm{~s}$

Step 4: $72^{\circ} \mathrm{C}$ for extension time (30-40 cycles from Step 2 to Step 4)

Step 5: $72^{\circ} \mathrm{C}$ for $10 \mathrm{~min}$

Annealing temperatures, extension times, and cycle numbers were modified depending on the target DNA. For genotyping PCR, RedTaq DNA polymerase (Sigma-Aldrich) was used.

\subsubsection{Site-Directed Mutagenesis}

The QuickChange II Site-Directed Mutagenesis Kit (Stratagene) was used according to the manufacturer's instructions with minor modifications. Briefly, the primer pair was designed in a fashion that the $5^{\prime}$-end is partially overlapped, and the mutation sites are located at the middle of the 
overlapping region. At least eight non-overlapping bases were introduced at the 3 '-end of the primer pair. 2 ng of plasmid DNA template was amplified in $25 \mu$ l of reaction mixtures containing oligonucleotide primers, dNTPs, and pfu DNA polymerase (Stratagene) in the appropriate reaction buffer. PCR reactions were carried out with basic cycle parameters as below:

Step 1: $94^{\circ} \mathrm{C}$ for $3 \mathrm{~min}$

Step 2: $94^{\circ} \mathrm{C}$ for $1 \mathrm{~min}$

Step 3: annealing temperature for $1 \mathrm{~min}$

Step 4: $72^{\circ} \mathrm{C}$ for extension time (18 cycles from Step 2 to Step 4)

Step 5: $55^{\circ} \mathrm{C}$ for $1 \mathrm{~min}$

Step 6: $72^{\circ} \mathrm{C}$ for $30 \mathrm{~min}$

Annealing temperatures and extension times were modified depending on the target plasmid size. The PCR product was then digested with Dpnl and transformed into $E$. coli. Plasmid DNA from the positive $E$. coli colonies was isolated and subjected to sequencing to confirm the mutation.

\subsubsection{Subcloning using the TOPO Cloning Kit}

PCR products were subcloned into the pCRII-TOPO vector using TOPO TA cloning Kits (Invitrogen) according to the protocol provided by the manufacturer. LB plates containing ampicillin or kanamycin, IPTG, and X-gal were used for the blue/white screening. Plasmid DNA from the white colonies were isolated and subjected to sequencing to confirm the sequence of insert DNA.

\subsubsection{RNA Preparation}

Cultured neurons were harvested at DIV8 with RIPA buffer, and the NucleoSpin RNA XS kit (Macherey-Nagel) was used according to the manufacturer's instructions. For polysome fractionation and mRNA analysis, $5 \times 10^{6}$ neurons were harvested at DIV8 with Lysis buffer $[10 \mathrm{mM}$ Tris/ $\mathrm{HCl} \mathrm{pH}$ 7.5, $10 \mathrm{mM} \mathrm{NaCl}, 10 \mathrm{mM} \mathrm{MgCl} 2,1 \%$ Triton X-100, 1\% deoxycholate, $1 \mathrm{mM}$ 
DTT, 30 units/ml RNase OUT (Invitrogen) containing $100 \mu \mathrm{g} / \mathrm{ml}$ cycloheximide (Sigma-Aldrich 01810), proteinase inhibitors (Sigma-Aldrich P8340), phosphatase inhibitor II (Sigma-Aldrich P5726), and phosphatase inhibitor III (Sigma-Aldirch P0044)]. After 5 min of incubation on ice, the extract was centrifuged for $8 \mathrm{~min}$ at $12,000 \times \mathrm{g}$ and $4^{\circ} \mathrm{C}$. The supernatant was loaded onto a $10-60 \%(w / v)$ sucrose gradient and sedimented by centrifugation at $4^{\circ} \mathrm{C}$ for $150 \mathrm{~min}$ at $169,000 \mathrm{xg}$ in a Beckman SW41 rotor.

RIPA buffer:

$50 \mathrm{mM}$ Tris- $\mathrm{HCl}\left(\mathrm{pH} 7.5\right.$ at $\left.4^{\circ} \mathrm{C}\right), 1 \mathrm{mM}$ EDTA, $150 \mathrm{mM} \mathrm{NaCl}, 1 \%$ Triton $X-$ $100,0.1 \%$ SDS

Lysis buffer:

$10 \mathrm{mM}$ Tris- $\mathrm{HCl}\left(\mathrm{pH} 7.5\right.$ at $\left.4^{\circ} \mathrm{C}\right), 10 \mathrm{mM} \mathrm{NaCl}, 10 \mathrm{mM} \mathrm{MgCl}, 1 \%$ Triton $X-$ 100, 1\% deoxycholate, 1 mM DTT, 30 units/ml RNase OUT (Invitrogen), 100 $\mu \mathrm{g} / \mathrm{ml}$ cycloheximide (Sigma-Aldrich 01810), proteinase inhibitors (SigmaAldrich P8340), phosphatase inhibitor II (Sigma-Aldrich P5726), and phosphatase inhibitor III (Sigma-Aldrich P0044)

\subsubsection{Real Time Quantitative-PCR (RT-qPCR)}

For the total mRNAs quantity study, $150 \mathrm{ng}$ of the total RNA were subjected to one cycle of retrotranscription in a mixture of random nonamer primers, anchored poly-dT primers, and the SuperScript III RNase H reverse transcriptase (Invitrogen) according to the manufacturer's instructions. Realtime PCR (RT-qPCR) was carried out with the SYBR Green PCR master mix (Applied Biosystems), cDNAs, and the gene specific primers. Samples were analyzed using the 7500 Fast Real-Time PCR System (Applied Biosystems). The input concentration of cDNAs was optimized to ensure that the detection was within the linear range. Relative levels of mRNAs of interest were normalized to the levels of two house-keeping mRNAs, Rp/13a and ATP5b.

For the RNA analysis (mRNA translation) after polysome fractionation, each gradient was collected into 12 fractions followed by the addition of $1 \%$ 
SDS (final concentration), $40 \mathrm{pg}$ of exogenous (in vitro transcribed) $B C 200$ RNAs, $10 \mathrm{mg}$ glycogen, and proteinase $\mathrm{K}(50 \mu \mathrm{g} / \mathrm{ml})$, and incubated for 30 min at $37^{\circ} \mathrm{C}$. The exogenous human $B C 200$ RNA (different sequences from the rodent $B C 1$ RNA) was used to monitor possible RNA loss from each fraction during RNA phenol/chloroform extraction and precipitation. RNAs were precipitated with $0.2 \mathrm{M} \mathrm{NaOAc}$ and 0.7 volume of isopropanol. Pellets were then re-suspended in $10 \mu \mathrm{l}$ of $\mathrm{ddH}_{2} \mathrm{O}$. The RNA fractions 1-7 (polysomal fraction, $\mathrm{P}$ ) and 1-12 (total RNA) were pooled. Following the in vitro retrotranscription the real-time PCR was performed. The translational efficiency was calculated as follows: $2^{-\left[\Delta \mathrm{Ct}(\mathrm{P})-\Delta \mathrm{Ct}\left(\text { Total }^{\prime}\right]\right.}=2^{-\Delta \Delta \mathrm{Ct}}$, where $\Delta \mathrm{Ct}$ equals Ct (specific mRNAs) - Ct (BC200 RNA).

\subsubsection{Cloning Strategies for Constructs Generated in This Study}

pCRII TOPO-p25

The cDNA fragment encoding human p25 was amplified by PCR from the pGEX6P-2-p35 vector using primers 28606/28607 and subcloned into the pCRII-TOPO vector.

\section{pClneoHA-p25}

The p25 cDNA was excised from the pCRII-TOPO-p25 vector using EcoRI/Xhol and ligated to the EcoRI/Sall sites of $\mathrm{pCIneoHA}$ vector.

\section{pClneoMyc-Cdk5}

The full length Cdk5 WT cDNA was excised from the pRaichuMyc-Cdk5 WT vectors using $E c o R I / N o t l$ and ligated to the corresponding sites of pCIneoMyc vector.

\section{pcDNA3.1nMyc-DEST}

Primers 30937/30938 were used to compose the cDNA encoding the Myctag with a N-terminal Hindlll site. The cDNA fragment was then digested with HindIII and ligated into the pDNA3.1nV5-DEST vector with the V5 cDNA fragment excised using HindIII/EcoRV. 
pcDNA3.1nMyc-Tubulin4A

pcDNA3.1nMyc-Uba1

pcDNA3.1nMyc-Prkar1b

pcDNA3.1nMyc-Map3k6

cDNAs from the corresponding Gateway entry vectors were transferred to the Gateway destination vector pcDNA3.1nMyc-DEST after LR reactions using the Gateway cloning method.

pGEX4T-1-WWP1 C886S

The C-terminal WWP1 C886S cDNA fragment was excised from the pCIneoMyc-WWP1 C886S vector using Xhol/Notl and ligated to the pGEX4T-1-WWP1 WT vector with the C-terminal WWP1 WT cDNA fragment excised using $\mathrm{Xhol} / \mathrm{Notl}$.

pGEX6P-1-Cdk5 WT

The full length Cdk5 WT cDNA fragment was excised from the pRaichuMycCdk5 WT vector using EcoRI/Xhol and ligated to the corresponding sites of GEX6P-1 vector.

pGEX6P-1-Cdk5 P234A, Y236F

Primers 31918/31919 were used to generate the pGEX6P-1-Cdk5 P234A, Y236F vector using the Quick Change site-directed mutagenesis method.

pCRII-TOPO-Myc-Cdk5

The cDNA encoding a N-terminal Myc-tagged Cdk5 WT was amplified by PCR using primers 27283/30007 from the pClneoMyc-Cdk5 WT vector and subcloned into the pCRII-TOPO vector.

pRRLsinPPT-CMV-WPRE-Myc-Cdk5 WT

The N-terminal Myc-tagged Cdk5 WT cDNA fragment were excised from the pCRII-TOPO-Myc-Cdk5 WT vector using BamHI, and ligated to the BamHI site of pRRLsinPPT-CMV-WPRE vector. 
pMalC2-p25

The p25 cDNA was excised from the pCRII-TOPO-p25 vector using EcoRI/Xhol and ligated to the EcoRI/Sall sites of pMalC2 vector.

pFUGW-WWP2 WT

The full length WWP2 cDNA was excised from the pCRII-TOPO-WWP2 WT vector using Spel/Xbal, and ligated into the pFUGW vector with the EGFP cDNA fragment excised by Xbal.

\section{pFUGW-WWP1 WT}

The full length WWP1 cDNA was excised from the pCIneoMyc-WWP1 WT vector using EcoRI/Mfel, and ligated into the pFUGW-WWP2 WT vector with the WWP2 WT cDNA excised by EcoRI.

\subsection{Biochemistry}

\subsubsection{Determination of Protein Concentration}

To quantify the protein concentration, the bicinchoninic acid (BCA) method was employed according to the manufacturer's instructions (Thermo Scientific). Briefly, protein samples were incubated with the BCA regents at $37^{\circ} \mathrm{C}$ for $30 \mathrm{~min}$ and the absorbance at $652 \mathrm{~nm}$ of each sample was measured. The standard BSA samples with known concentration were referred to in order to estimate the protein concentration of each sample.

\subsubsection{Sodium dodecyl sulfate polyacrylamide gel electrophoresis (SDS-PAGE)}

To separate proteins based on their molecular sizes, protein samples were subjected to SDS-PAGE under the denatured condition. Briefly, protein samples were dissolved in Laemmili buffer, followed by boiling at $95^{\circ} \mathrm{C}$ for 3 min. The SDS-PAGE gel consisting of the upper part stacking gel and the lower part resolving gel (see recipes below) was prepared and set on the Bio-Rad Mini-PTOTEAN 251 casting system. Protein samples were loaded 
onto the gel and electrophoresis was performed with a constant current of 20 $\mathrm{mA}$ for samples in the stacking gel and 30-40 mA for samples in the resolving gel. Generally, 5-20 $\mu$ g of protein was loaded alongside an SDSPAGE ladder (Invitrogen).

\section{Laemmili Buffer:}

10\% Glycerol, $50 \mathrm{mM}$ Tris- $\mathrm{HCl}$ (pH 6.8 at RT), 2 mM EDTA, 2\% SDS, 100 mM DTT, $0.05 \%$ Bromophenol blue.

Upper stacking gel:

$5 \%$ acrylamide/ $N, N$-Methylene-bis-Acrylamide (29:1) Solution (AMBA), 125 $\mathrm{mM}$ Tris- $\mathrm{HCl}$ ( $\mathrm{pH} 6.8$ at RT), $0.1 \%$ SDS, $0.05 \%$ ammonium persulfate (APS), $0.005 \%$ TEMED.

Lower resolving gel:

8-15\% AMBA, $325 \mathrm{mM}$ Tris- $\mathrm{HCl}$ (pH 8.8), $0.1 \%$ SDS, $0.05 \%$ APS, $0.005 \%$ TEMED.

Running buffer:

$25 \mathrm{mM}$ Tris- $\mathrm{HCl}, 250 \mathrm{mM}$ Glycine, $0.1 \%$ SDS (pH 8.8).

\subsubsection{Western Blotting}

Proteins separated by SDS-PAGE were electrically transferred to PVDF or nitrocellulose membranes. Non-specific binding of antibodies to the membranes was pre-blocked by incubation with $5 \%$ skimmed milk in TBST containing $5 \%$ goat serum. Subsequently, the membranes were incubated with primary antibodies, followed by incubation with the corresponding secondary antibodies diluted in 5\% skimmed milk in TBST. Signals were detected with the enhanced chemiluminescence (ECL) system (GE Healthcare), or the Odyssey Infrared Imaging System (Li-COR Biosciences). Quantification was done by using the Odyssey software, the Bio-Rad ChemiDoc system, or ImageJ. 
TBST:

$20 \mathrm{mM}$ Tris- $\mathrm{HCl}$ (pH 7.5 at RT), $137 \mathrm{mM} \mathrm{NaCl}, 1 \%$ Tween20 (w/v).

\subsubsection{Purification of Recombinant GST-Fusion Proteins}

The GST gene fusion system (GE Healthcare Life Sciences) was employed to purify GST-fusion proteins. cDNA fragments encoding the fulllength WWP1 WT, WWP1 C886S, WWP2 WT, Cdk5 WT, and Cdk5 $\mathrm{P} 236 \mathrm{~A} / \mathrm{Y} 238 \mathrm{~F}$ were subcloned into pGEX-4T-1 or pGEX-6P-1 vectors, which were then back-transformed into the BL21 Rossetta $E$. coli strain. A single colony was inoculated in $50 \mathrm{ml}$ LB medium in the presence of ampicillin $(\mathrm{LB}+\mathrm{Amp})$ and pre-cultured at $37^{\circ} \mathrm{C}$ overnight. The pre-culture was then transferred into $1000 \mathrm{ml}$ of LB+Amp medium and stirred at $17^{\circ} \mathrm{C}$ to allow for E. coli growth. When the optical density at $600 \mathrm{~nm}\left(\mathrm{OD}_{600}\right)$ of the E.coli culture reached 0.5-0.6, expression of the GST-fusion proteins were induced by the addition of $0.1 \mathrm{mM}$ isopropyl- $\beta-D-1$-thiogalactopyranoside (IPTG). $E$. coli cells were harvested by centrifugation at $3500 \times \mathrm{g}$ at $4^{\circ} \mathrm{C}$ for $30 \mathrm{~min}$ when the $\mathrm{OD}_{600}$ reached $0.9-1.0$. The pellet was then resuspended in $50 \mathrm{ml}$ Lysis buffer and evenly distributed into ten aliquots. The aliquots were flash frozen in liquid nitrogen and stored at $-80^{\circ} \mathrm{C}$ until further purification steps.

For further purification of the GST-fusion proteins, bacteria aliquots were thawed in Lysis buffer and supplemented with protease inhibitors (0.2 $\mathrm{mM}$ PMSF, $1 \mu \mathrm{g} / \mathrm{ml}$ Aprotinin, $0.5 \mu \mathrm{g} / \mathrm{ml}$ Leupeptin), lysozyme (1 $\mathrm{mg} / \mathrm{ml}$ ), DNase I $(50 \mu \mathrm{g} / \mathrm{ml}), \mathrm{MgCl}_{2}(1 \mathrm{mM})$ and DTT $(1 \mathrm{mM})$. After $10 \mathrm{~min}$ incubation on ice, the cells were sonicated for $20 \mathrm{~s}$ for three times with 1 min interval of incubation on ice, followed by the addition of $0.8 \%$ CHAPS to solubilize the proteins. After $30 \mathrm{~min}$ incubation on ice with gentle agitation, insoluble fractions were removed by centrifugation at $10,000 \times \mathrm{g}$ for $15 \mathrm{~min}$ at $4^{\circ} \mathrm{C}$. The GST-fusion proteins were affinity-purified by incubation with the reduced glutathione (GSH) covalently coupled to sepharose 4B beads (GE Healthcare) according to the manufacturer's instructions. The bound fractions 
of GST-fusion proteins were then eluted with $40 \mathrm{mM}$ reduced $\mathrm{GSH}$ or incubated with thrombin or prescission protease to remove the GST tag.

Lysis buffer A (for GST-WWP1 WT, GST-WWP1 C886S, GST-WWP2 WT): $20 \mathrm{mM}$ Tris- $\mathrm{HCl}\left(\mathrm{pH} 7.5\right.$ at $\left.4^{\circ} \mathrm{C}\right), 150 \mathrm{mM} \mathrm{NaCl}, 1 \mathrm{mM}$ EDTA, $1 \mathrm{mM}$ DTT Lysis buffer B (for GST-Cdk5 WT, GST-Cdk5 P234A,Y236F): $20 \mathrm{mM}$ MOPS (pH 7.5 at $4^{\circ} \mathrm{C}$ ), $150 \mathrm{mM} \mathrm{NaCl}, 1 \mathrm{mM}$ EDTA, $1 \mathrm{mM}$ DTT

\subsubsection{Affinity Purification of GST-WWP1 Binding Proteins}

The recombinant GST-WWP1 WT was purified from E. coli and $50 \mu \mathrm{g}$ of the purified GST-WWP1 WT was immobilized on $50 \mu \mathrm{l}$ glutathione sepharose $4 \mathrm{~B}$ beads (GE Healthcare Life Sciences). After washing the beads for three times with five bed volumes of A-buffer, a 0.8\% CHAPS extract (in A-Buffer) of mouse brain synaptosomes was loaded to the beads, which were then washed for three times with five bed volumes of A-buffer. Subsequently, the GST-WWP1 binding proteins were eluted with B-buffer (same composition as A-buffer except for $1 \mathrm{M} \mathrm{NaCl}$ ), followed by elution with A-buffer containing $40 \mathrm{mM}$ glutathione.

A-buffer:

$20 \mathrm{mM}$ Tris- $\mathrm{HCl}\left(\mathrm{pH} 7.5\right.$ at $\left.4^{\circ} \mathrm{C}\right), 0.32 \mathrm{M}$ Sucrose, $1 \mathrm{mM}$ EDTA, $1 \mathrm{mM}$ DTT, and $1 \%$ Triton X-100, $150 \mathrm{mM} \mathrm{NaCl}, 0.2 \mathrm{mM}$ PMSF, $1 \mu \mathrm{g} / \mathrm{ml}$ Aprotinin, 0.5 $\mu \mathrm{g} / \mathrm{ml}$ Leupeptin.

B-buffer:

$20 \mathrm{mM}$ Tris- $\mathrm{HCl}\left(\mathrm{pH} 7.5\right.$ at $\left.4^{\circ} \mathrm{C}\right), 0.32 \mathrm{M}$ Sucrose, $1 \mathrm{mM}$ EDTA, $1 \mathrm{mM}$ DTT, and $1 \%$ Triton X-100, $1 \mathrm{M} \mathrm{NaCl}, 0.2 \mathrm{mM} \mathrm{PMSF}, 1 \mu \mathrm{g} / \mathrm{ml}$ Aprotinin, $0.5 \mu \mathrm{g} / \mathrm{ml}$ Leupeptin. 


\subsubsection{Protein Identification by Mass Spectrometry}

Proteins eluted from the GST-WWP1 beads were separated by SDSPAGE under the reduced condition on a pre-cast NuPAGE 4\%-10\% Bis-Tris gradient gel (Invitrogen) using a MOPS-based buffer system recommended by the manufacturer. Eluates from GST beads and GST-WWP1 beads with only buffer input were used as negative controls. After colloidal Coomassie staining, gel plugs were manually excised from individual bands that are specifically enriched in the eluates from GST-WWP1 beads with brain lysates input. Proteins in the gel were then extracted, trypsinized, and subjected to an automated platform for the identification of peptides using mass spectrometry as described previously (Jahn et al., 2006; Reumann et al., 2007; Werner et al., 2007). An Ultraflex MALDI-TOF-mass spectrometer (Bruker Daltonics) was used to acquire both peptide mass fingerprint and fragment ion spectra, resulting in confident peptide identifications based on the mass and the sequence information. The resulting information was then analyzed by Mascot software (Matrix Science) to match the peptide sequences with Swiss-prot and NCBI databases (Taxonomy: Mouse) using the parameter settings described earlier (Jahn et al., 2006; Reumann et al., 2007; Werner et al., 2007). Only the proteins identified with high confidence in both databases were taken into account as potential binding partners of WWP1.

\subsubsection{In Vitro Binding Assay}

$50 \mu \mathrm{g}$ of recombinant GST-WWP1 WT and GST-WWP2 WT, or $45 \mu \mathrm{g}$ of GST-Cdk5 WT and GST-Cdk5 P234A/Y236F were purified from E. coli and immobilized on $50 \mu \mathrm{l}$ of glutathione sepharose 4B beads (GE Healthcare Life Sciences) in columns. Proteins of interest were purified from HEK293FT cells or $E$. coli and loaded to the columns. After extensively washing of the columns with the corresponding binding buffer, the beads were collected and subjected to Western blotting analysis. 
Binding buffer A:

$20 \mathrm{mM}$ Tris- $\mathrm{HCl}\left(\mathrm{pH} 7.5\right.$ at $\left.4^{\circ} \mathrm{C}\right), 150 \mathrm{mM} \mathrm{NaCl}, 1 \mathrm{mM}$ EDTA, $0.32 \mathrm{M}$ Sucrose, 0.8\% CHAPS, $1 \mathrm{mM}$ DTT, $0.2 \mathrm{mM}$ PMSF, $1 \mu \mathrm{g} / \mathrm{ml}$ Aprotinin, 0.5 $\mu \mathrm{g} / \mathrm{ml}$ Leupeptin.

Binding buffer B (for Cdk5):

$20 \mathrm{mM}$ MOPS (pH 7.5 at $4^{\circ} \mathrm{C}$ ), $150 \mathrm{mM} \mathrm{NaCl}, 1 \mathrm{mM}$ EDTA, $1 \mathrm{mM}$ DTT, 0.2 $\mathrm{mM}$ PMSF, $1 \mu \mathrm{g} / \mathrm{ml}$ Aprotinin, $0.5 \mu \mathrm{g} / \mathrm{ml}$ Leupeptin.

\subsubsection{In Vitro Ubiquitination Assay}

The in vitro ubiquitination reactions were carried out in $20 \mu$ l of the reaction mixture containing $2 \mathrm{mM}$ ATP, $1 \mu \mathrm{g}$ ubiquitin (Boston Biochem), 20 ng UBE1 (Boston Biochem), 200 ng of each E2 (UbcH5b, UbcH6, and UbcH7; Boston Biochem), 250 ng of the recombinant E3 purified from E. coli (WWP1 WT, WWP1 C886S or WWP2 WT), and 100-400 ng of the substrate of interest (either purified from E.coli or immunopurified from transfected HEK293FT cells) in a Tris-based buffer [50 mM Tris- $\mathrm{HCl}\left(\mathrm{pH} 8.0\right.$ at $\left.25^{\circ} \mathrm{C}\right)$, $150 \mathrm{mM} \mathrm{NaCl}, 10 \mathrm{mM} \mathrm{MgCl} 2$ ], and incubated at $37^{\circ} \mathrm{C}$ for $1 \mathrm{~h}$ with gentle agitation. Reactions were then stopped by the addition of Laemmili buffer, and the results were analyzed by Western blotting.

\subsubsection{Immunoprecipitation (IP)}

For anti-Myc IP, agarose beads conjugated with the anti-Myc antibody (Sigma-Aldrich) were used. For IP of other proteins, Protein A sepharose CL4B beads (GE Healthcare) were incubated with 10\% BSA for 30 min at RT, followed by washing twice with PBS and equilibrating in the corresponding lysis buffer containing appropriate detergent(s). Antibody-antigen complexes were bound to Protein A sepharose CL-4B beads at $4^{\circ} \mathrm{C}$. After extensively washing the beads with lysis buffer, proteins were eluted by boiling in Laemmli buffer, followed by SDS-PAGE and analysis of the results using Western blotting. For immunoprecipitation of proteins under denaturing 
conditions, proteins were extracted with lysis buffer containing $1 \%$ SDS, boiled for $5 \mathrm{~min}$, and the SDS was neutralized with lysis buffer containing $1 \%$ TritonX-100.

\subsubsection{In Vivo Ubiquitination Assay}

Mammalian expression vector for Myc-Cdk5 was co-transfected with WWP1 WT or WWP2 WT expression vectors and either HA-ubiquitin WT, ubiquitin K48R, or ubiquitin K63R expression vectors using Lipofectamin2000 (Invitrogen). Cells were harvested in A-buffer containing $0.5 \%$ SDS and the lysates were boiled for $5 \mathrm{~min}$, followed by neutralizing the SDS by diluting five times with A-buffer containing 1\% TritonX-100. Myc-Cdk5 was then immuoprecipitated using the anti-Myc agarose beads (Sigma-Aldrich). After extensively washing the beads with lysis buffer containing $1 \%$ TritonX-100, the immuoprecipitated Myc-Cdk5 was subjected to SDS-PAGE, followed by Western blotting to HA or ubiquitin.

A-buffer:

$20 \mathrm{mM}$ Tris- $\mathrm{HCl}\left(\mathrm{pH} 7.5\right.$ at $\left.4^{\circ} \mathrm{C}\right), 150 \mathrm{mM} \mathrm{NaCl}, 1 \mathrm{mM}$ EDTA, $0.2 \mathrm{mM}$ PMSF, $1 \mu \mathrm{g} / \mathrm{ml}$ Aprotinin, $0.5 \mu \mathrm{g} / \mathrm{ml}$ Leupeptin, $10 \mathrm{mM}$ NEM, 20 mM Indoacetamide.

\subsubsection{PTEN Phosphatase Activity Assay}

PTEN was immunoprecipitated from P7 mouse brains in lysis buffer. The activity of the immunoprecitated PTEN was assessed using a commercially available phosphatase assay kit (K-1500, Echelon) according to the manufacturer's instructions. Briefly, protein A sepharose CL-4B beads with immunoprecipitated PTEN were washed with reaction buffer. Enzyme reactions were carried out by incubating PTEN with $120 \mu \mathrm{M}$ of water soluble PtdlnsP $\mathrm{P}_{3}\left(\mathrm{diC}_{8}\right.$, Echelon) in the PTEN reaction buffer for $40 \mathrm{~min}$ at $37^{\circ} \mathrm{C}$. Free phosphate generated was detected by the Malachite Green reagent supplied in the kit in a colorimetric manner. Absorbance at $650 \mathrm{~nm}$ was measured and the free phosphate level $(\mathrm{pmol})$ was calculated using a phosphate standard. 
The percentage of Ptdlns $\mathrm{P}_{3}$ conversion was calculated according to the following formula: $\%$ PtdlnsP $_{3}$ conversion $=\{[($ free phosphates in reaction, pmol) - (background phosphate in substrate only control, pmol)] $\div$ (input PtdlnsP $\left.\left.\mathrm{P}_{3}, \mathrm{pmol}\right)\right\} \times 100 \%$.

Lysis buffer:

$25 \mathrm{mM}$ Tris/ $\mathrm{HCl} \mathrm{pH}$ 8.0, $150 \mathrm{mM} \mathrm{NaCl}, 1 \%$ TritonX-100, $1 \mathrm{mM}$ EDTA, 0.2 $\mathrm{mM}$ PMSF, $1 \mu \mathrm{g} / \mathrm{ml}$ Aprotinin, $0.5 \mu \mathrm{g} / \mathrm{ml}$ Leupeptin.

Reaction buffer:

$25 \mathrm{mM}$ Tris/ $\mathrm{HCl} \mathrm{pH} \mathrm{7.5,} 140 \mathrm{mM} \mathrm{NaCl}, 2.7 \mathrm{mM} \mathrm{KCl}, 10 \mathrm{mM}$ DTT.

\subsection{Cell Biology}

\subsubsection{Culture Media and Solutions}

10\% FCS/DMEM:

$500 \mathrm{ml}$ DMEM, $50 \mathrm{ml}$ FCS, Glutamine (Invitrogen), $5 \mathrm{ml}$ Penicillin/Streptomycin (100x, Invitrogen).

Complete Neurobasal medium:

$500 \mathrm{ml}$ Neurobasal A, $5 \mathrm{ml}$ GlutaMAX I (Invitrogen), $10 \mathrm{ml} \mathrm{B-27}$ supplement (Invitrogen), $1 \mathrm{ml}$ Penicillin/Streptomycin (100x, Invitrogen).

\section{Papain solution:}

20-25 units of Papain per $1 \mathrm{ml}$ of solution 1 were added and bubbled with carbogen (95\% oxygen, $5 \%$ carbon dioxide) for $10-20$ min until the solution becomes clear. The Papain Solution was sterilized using a $0.22 \mu \mathrm{m}$ filter (Millipore) and kept on the ice before use.

Stop solution:

$2.5 \mathrm{mg} / \mathrm{ml} \mathrm{BSA}, 2.5 \mathrm{mg} / \mathrm{ml}$ trypsin inhibitor, and 10\% FCS in DMEM. The solution was pre-warmed at $37^{\circ} \mathrm{C}$ before use. 


\subsubsection{Primary Mouse Hippocampal Culture Preparation}

Hippocampi from P0 mice were isolated and digested in 25 units $/ \mathrm{ml}$ of papain (Worthington Biochemical) in papain solution at $37^{\circ} \mathrm{C}$ for $1 \mathrm{~h}$, followed by incubation in stop solution at $37^{\circ} \mathrm{C}$ for $15 \mathrm{~min}$. The papain digested hippocampi were then mechanically triturated with a plastic tip for P-200 Pipetman in $200 \mu \mathrm{l}$ of the pre-warmed complete Neurobasal medium. The dissociated neurons were plated on poly-L-lysine (Sigma-Aldrich) coated coverslips at a density of 30,000-60,000 cells per $\mathrm{cm}^{2}$. Neurons were cultured in the complete Neurobasal medium at $37^{\circ} \mathrm{C}$ in a cell culture incubator with $5 \% \mathrm{CO}_{2}$ (Thermo) before harvesting or fixation.

\subsubsection{Transfection of Primary Hippocampal Culture}

Cultured hippocampal neurons were transfected by the calcium phosphate method at DIV1. Briefly, the culture medium in each well of the 24-well plate was replaced with $500 \mu \mathrm{l}$ of pre-warmed Opti-MEM I (Invitrogen) $30 \mathrm{~min}$ before transfection. $45 \mu \mathrm{l}$ of Transfection mixture containing $3 \mu \mathrm{g}$ of DNA and $25 \mathrm{mM} \mathrm{CaCl}_{2}$ was prepared in a $1.5 \mathrm{ml}$ eppendorf, and $45 \mu \mathrm{l}$ of $2 x$ HBS was added to the mixture drop-wisely with gentle shake. After incubation at room temperature for at least $20 \mathrm{~min}, 30 \mu \mathrm{l}$ of the mixture was applied to each well of the 24-well plate. The neurons were incubated in a $37^{\circ} \mathrm{C} / 5 \% \mathrm{CO}_{2}$ incubator for $25 \mathrm{~min}$, followed by removing the calcium phosphate precipitates by washing with the Neurobasal $A$ medium pre-equilibrated in a $37^{\circ} \mathrm{C} / 10 \% \mathrm{CO}_{2}$ incubator. The neurons were further cultured in the complete Neurobasal medium at $37^{\circ} \mathrm{C}$ in a cell culture incubator with $5 \% \mathrm{CO}_{2}$ before harvesting or fixation.

2x HBS:

$274 \mathrm{mM} \mathrm{NaCl}, 10 \mathrm{mM} \mathrm{KCl}, 1.4 \mathrm{mM} \mathrm{Na} \mathrm{HPO}_{4}, 15 \mathrm{mM}$ glucose, $42 \mathrm{mM}$ HEPES (Sigma, H3375, pH 7.08 adjusted by $\mathrm{NaOH}$ ). Aliquots were stored at $-20^{\circ} \mathrm{C}$. 


\subsubsection{HEK293FT Cell Line}

HEK293FT cells were maintained in tissue culture dishes with $10 \%$ FCS/DMEM in the $37^{\circ} \mathrm{C} / 5 \% \mathrm{CO}_{2}$ incubator. Transfection of HEK293FT cells was done by using Lipofectamine2000 (Invitrogen) according to the manufacturer's instructions.

\subsubsection{Lentivirus Preparation}

$1.6 \times 10^{7}$ HEK293FT cells (Invitrogen) were plated on a poly-L-Lysine (Sigma-Aldrich) coated 15-cm plastic dish and cultured for $24 \mathrm{~h}$ in OPTIMEM medium (Gibco) containing 10\% fetal calf serum (PAA). The packaging vector pCMVdeltaR8.2, the VSV-G expression vector pMD2.G, and the backbone vector were co-transfected to HEK293FT cells with Lipofectamine2000 (Invitrogen) according to the manufacturer's instructions. Cells were incubated for $18 \mathrm{~h}$, and afterwards the medium was changed to Dulbecco's modified Eagle medium (Gibco) containing 2\% fetal calf serum (PAA), 10 units $/ \mathrm{ml}$ penicillin, $10 \mu \mathrm{g} / \mathrm{ml}$ streptomycin (Gibco), and $10 \mathrm{mM}$ sodium butyrate (Merck). The culture medium, which contained lentiviral particles, was harvested $72 \mathrm{~h}$ after transfection. To increase the viral titer, Amicon Centrifugal Filters (100 kDa, Millipore) were used according to the manufacturer's instructions to concentrate viral particles. The high-titer lentivirus sample was then dialyzed with dialysis buffer overnight, flash frozen with liquid nitrogen, and stored in $-80^{\circ} \mathrm{C}$ until used.

Dialysis buffer:

$20 \mathrm{mM}$ Tris/ $\mathrm{HCl} \mathrm{pH} 8.0$ at $4^{\circ} \mathrm{C}, 150 \mathrm{mM} \mathrm{NaCl}$

\subsubsection{Immunocytochemistry (ICC)}

Cells cultured on the coverslips were fixed with $4 \%$ paraformaldehyde $(w / v) / P B S$ at $4^{\circ} \mathrm{C}$ for 20 minutes. After washing with PBS for three times, the cells were incubated with blocking buffer at room temperature for $1 \mathrm{~h}$, followed by incubation with primary antibodies diluted in blocking buffer at 
$4^{\circ} \mathrm{C}$ overnight. Subsequently, cells were washed three times with PBS and incubated with the corresponding fluorescently-labeled secondary antibodies diluted in blocking buffer at room temperature for $2 \mathrm{~h}$. After washing the cells with PBS for three times and once with $\mathrm{dd}_{2} \mathrm{O}$, coverslips were air-dried and mounted on the microscope glass slide with the mounting medium (Thermo Scientific).

Blocking buffer:

PBS containing 10\% Horse serum, 2\% BSA, 5\% (w/v) Sucrose, 0.3\% (w/v) TritonX-100

\subsection{Histology}

\subsubsection{In Utero Electroporation}

At embryonic stage 15.5 (E15.5), plasmid DNA was transfected to a subpopulation of neuronal progenitor cells, which generate mainly layer II/III cortical neurons. The electroporated brains were fixed at P21, when the axons of layer II/III cortical neurons have reached the contralateral side (callosal projections) and branched extensively into the somatosensory cortex. Pregnant mice were deeply anesthetized with isoflurane and placed on a warming pad $\left(31-32^{\circ} \mathrm{C}\right)$ throughout the surgery. An approximately $2-\mathrm{cm}$ midline incision in the abdomen was made, and uterine horns were drawn out through the incision and moistened with warmed PBS containing antibiotics (Penicillin-Streptomycin, Sigma-Aldrich). Plasmid DNA solution prepared with the EndoFree Plasmid Maxi Kit (Qiagen) was injected into the lateral ventricle through a glass micropipette, and electric pulses $(35 \mathrm{~V}, 50$ $\mathrm{mA}, 50 \mathrm{~ms}$ duration, $950 \mathrm{~ms}$ intervals, 8 pulses per embryo) were applied with the ECM830 electroporator (BTX Harvard Apparatus). The incision was then closed with surgical sutures. Animals were kept warm $\left(31-32^{\circ} \mathrm{C}\right)$ until they had recovered from anesthesia. 


\subsubsection{Perfusion}

For histological experiments using mouse brains, mice were perfused transcardially with PBS, followed by $4 \%$ paraformaldehyde $(\mathrm{w} / \mathrm{v}) / \mathrm{PBS}$. The brains were then taken out and post-fixed in $4 \%$ paraformaldehyde (w/v)/PBS at $4^{\circ} \mathrm{C}$ overnight. After washing twice with PBS, the brains were subjected to further histological experiments.

\subsubsection{Immunohistochemistry (IHC)}

Paraffin embedded mouse brain coronal sections were used for the anti-NeuN staining. Paraffin sections $(5 \mu \mathrm{m})$ were collected on glass slides. After de-paraffination, sections were incubated in blocking buffer (20\% goat serum in $10 \%$ BSA/PBS), and subsequently incubated with primary antibodies at $4^{\circ} \mathrm{C}$ overnight, followed by incubation with the corresponding fluorescently-labeled secondary antibodies. Images were acquired on an Olympus BX61 microscope. Numbers of cells were counted manually by a blind observer with the help of the 'Cell Counter' plugin in ImageJ. For analyses of axon morphogenesis in vivo, immunostaining of $100 \mu \mathrm{m}$ floating vibratome sections was performed. Brain sections were blocked with blocking buffer (5\% goat serum, $0.1 \%$ Triton X-100 in PBS), followed by incubation with chicken anti-GFP (Aves Lab), rabbit anti-Cux1 (Santa Cruz), and mouse anti-Cre (Sigma-Aldrich) antibodies that had been diluted in the same buffer. The sections were then incubated with fluorescently labeled secondary antibodies diluted in $2 \%$ goat serum/PBS.

\subsection{Image Analysis and Statistic}

To study the outgrowth of axons, neurons were transfected with an EGFP expression vector at DIV1, fixed at DIV7, immunostained for GFP (7.1 and 13.1, Roche), and pictures of EGFP-expressing neurons were acquired with a 10x objective on an Olympus BX61 fluorescence microscope. Analyses were performed using the NeuronJ plugin in ImageJ (NIH). The 
longest neurite was taken as the axon, and the branch number was determined by counting the number of neurites $(>15 \mu \mathrm{m})$ extending from the longest neurite. For studies on the intracellular localization of PTEN, neurons were fixed at DIV7 and subjected to immunostaining for PTEN. The fluorescence staining was imaged with a $63 \times$ objective lens on a Leica confocal microscope (TCS SP2 equipped with AOBS). The mean intensity of PTEN signals was scored using ImageJ. Nuclei were defined by DAPI staining, and the dimension of neuronal somata was determined manually. For analyses of neuronal cell polarity, AnkyrinG-positive but MAP2-negative neurites were scored as axons. For Sholl analysis of neurite structure, neurons were transfected with an EGFP expression vector at DIV1. The neurons were then treated with $20 \mathrm{nM}$ rapamycin (Cell Signaling) or DMSO at DIV6 and fixed at DIV10. The 'Advanced Sholl Analysis' plugin in ImageJ was used for analysis, in which concentric circles were drawn at $7.5 \mu \mathrm{m}$ intervals using the cell body as the common center, and the numbers of neurites crossing each of the circles were counted. For all of the morphological analyses on cultured neurons, neurons were chosen because they are healthy, with continuous and extended neurites. Inclusion of neurons with neurites circling around the soma was avoided, since this morphology complicates the analysis. Acquisition and analyses of images were done by a blind observer.

For quantification of axon branching in vivo, fluorescence images were acquired on a Leica SP2 confocal microscope. For each brain section, a zstack serial scan for 10 images at $2.5 \mu \mathrm{m}$ intervals was performed at the approximate level of $0.26 \mathrm{~mm}$ from the bregma. Images taken from the somatosensory cortex at the contralateral side were then projected to two dimensions using the maximum intensity projection function of the Leica confocal software. Signals from the cortical surface (cortical level set as 1 ) to the bottom of the white matter (cortical level set as 0 ) were quantified using the 'plot profile' function in ImageJ. Fluorescence intensity was normalized to the maximum value in the white matter to compensate for the variability in numbers of afferent axons originating from the transfected neurons. The 
normalized maximum values from the upper layer (cortical level 0.7-1) and the lower layer (cortical level 0.25-0.69) of the cortex were subjected to statistical analyses.

Statistical analyses were done using the Graphpad Prism 5 (Graphpad Software). A statistical significant difference was concluded when the $p$-value was less than 0.05 . The following annotations were used in the present study to indicate the $p$-value: ${ }^{*} p<0.05 ;{ }^{* *} p<0.01 ;{ }^{* *} p<0.001$. 


\section{Results}

\subsection{Ubiquitin E3 Ligase Nedd4-1 Acts as a Downstream Target of PI3K/PTEN-mTORC1 Signaling to Promote Neurite Growth (Hsia et al., 2014)}

\subsubsection{KO of Nedd4-1 and Nedd4-2 Causes Defects in Axonal Growth}

Mammalian Nedd4-1 promotes the growth and arborization of dendrites by monoubiquitinating Rap2 (Kawabe et al., 2010). In an independent study, Xenopus laevis Nedd4, which is most homologous to mammalian Nedd4-2, was reported to control axonal branching in retinal ganglion cells by polyubiquitinating PTEN (Drinjakovic et al., 2010). Consistent with the latter study, I found that deletion of Nedd4-2 in cultured hippocampal neurons resulted in reduced neurite complexity (Figure 3-1). In order to avoid possible compensation of the functions of Nedd4-1 by Nedd4-2 and to study the roles of Nedd4-1/Nedd4-2 in axonal development in mammalian neurons, I characterized postmitotic neuron-specific Nedd4-1 and Nedd4-2 conditional double KO mice (NEX-Cre;Nedd4-1 ${ }^{\mathrm{f} / \mathrm{f}} ; \mathrm{Nedd4}-2^{\mathrm{f} / \mathrm{f}}$, NEX-N1/2 $2^{\mathrm{f} / \mathrm{f}}$ ). The NEX$\mathrm{N} 1 / 2^{\mathrm{f} / \mathrm{f}}$ mice are viable and show no obvious behavioral alterations in the cage environment. However, macroanatomical analyses of developing NEX-N1/2 ${ }^{\mathrm{f} / \mathrm{f}}$ mouse brains at postnatal day 16 (P16) showed a reduction in the thickness of the cortex while the total number of neurons was not changed (Figure 3-2). Therefore, this reduction is likely caused by the reduced length of dendrites that was reported previously (Kawabe et al., 2010).

To study the roles of Nedd4-1 and Nedd4-2 in axon morphogenesis in vitro, I prepared primary cultured hippocampal neurons from control or NEX$\mathrm{N} 1 / 2^{\mathrm{f} / \mathrm{f}}$ mice and transfected them with an EGFP expressing vector to visualize neurite structures. At day-in-vitro 7 (DIV7), the length of the main axon shaft and the number of primary axonal branches showed a significant reduction in cultured NEX-N1/2/f neurons relative to controls (Figure 3-3 AC). In an independent set of experiment, I characterized the axonal 
morphology of cultured hippocampal neurons prepared from NEX-Cre WT or $N E X$-Cre heterozygote (Het) mice (Figure 3-3 D-F). No difference in the axonal morphology was observed between these two groups, indicating that NEX-Cre expression itself does not affect axon morphogenesis and the reduced axonal growth of NEX-N1/2f/f neurons (Figure 3-3 A-C) is a specific outcome of Nedd4-1 and Nedd4-2 deletion.
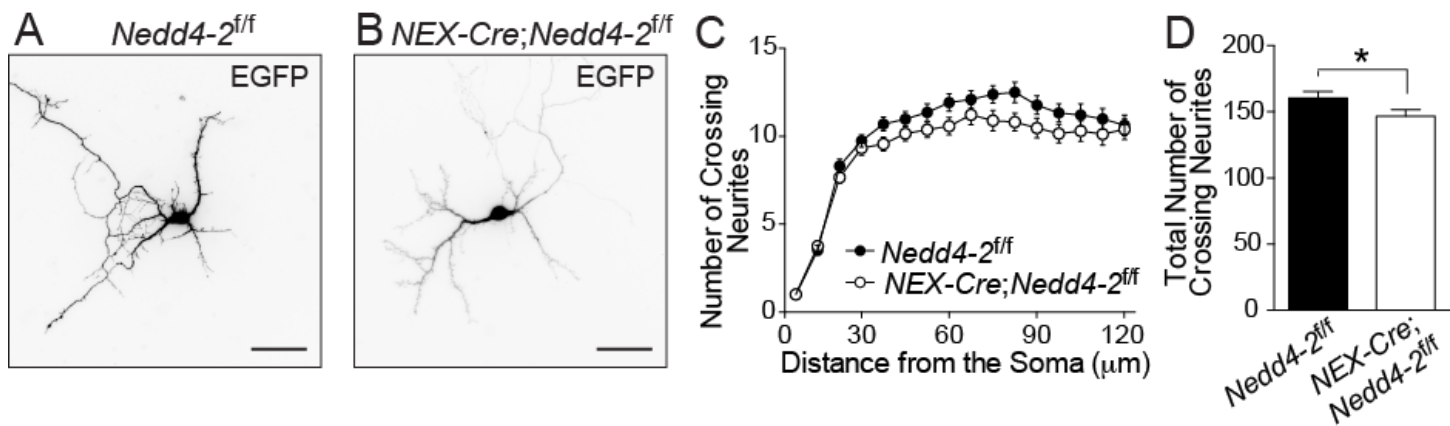

Figure 3-1. Nedd4-2 Regulates Neurite Growth in Mouse Hippocampal Neurons

( $A$ and $B$ ) Representative images of control $(A)$ and Nedd4-2 KO $(B)$ cultured hippocampal neurons transfected with an EGFP expressing vector. Scale bars, $50 \mu \mathrm{m}$. (C) Sholl analyses for the two groups in $(A)$ and $(B)$. Note that $\mathrm{KO}$ of Nedd4-2 slightly reduced neurite growth. $(D)$ Statistical analysis of the total number of crossing neurites obtained in the Sholl analysis shown in (C). Nedd4- ${ }^{\mathrm{fff}}, \mathrm{N}=87 ; \mathrm{NEX}$-Cre;Nedd4-2/f, $\mathrm{N}=73 .{ }^{*} p=0.0433$, unpaired $t$ test. Data are expressed as mean \pm SEM. 


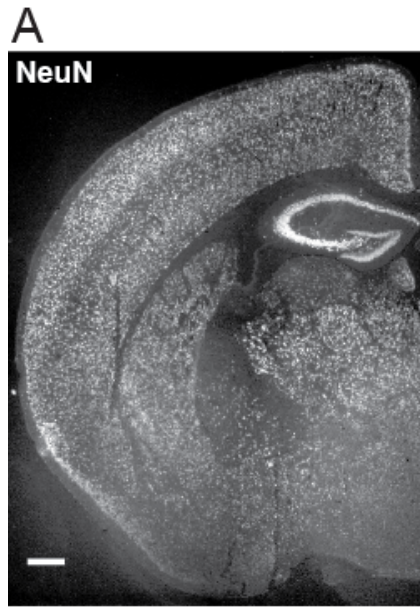

Nedd4-1 $1^{\mathrm{f} / \mathrm{f}} ;$ Nedd4-2 $2^{\mathrm{f} / \mathrm{f}}$

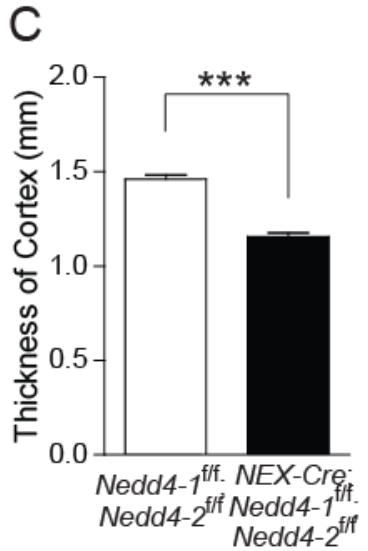

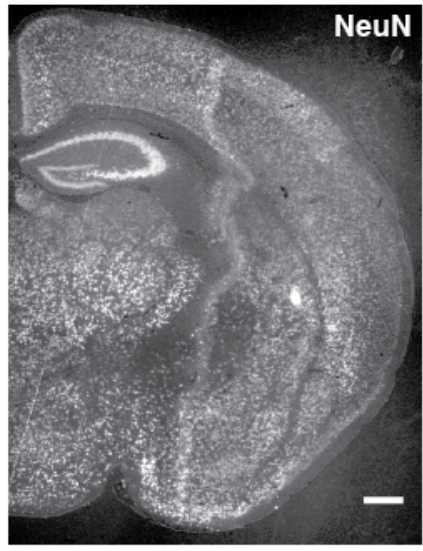

NEX-Cre;Nedd4-1 $1^{\mathrm{f} / \mathrm{f}} ;$ Nedd4-2 ${ }^{\mathrm{f} / \mathrm{f}}$
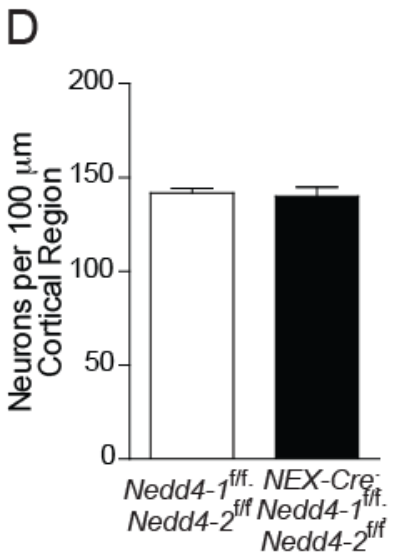

B

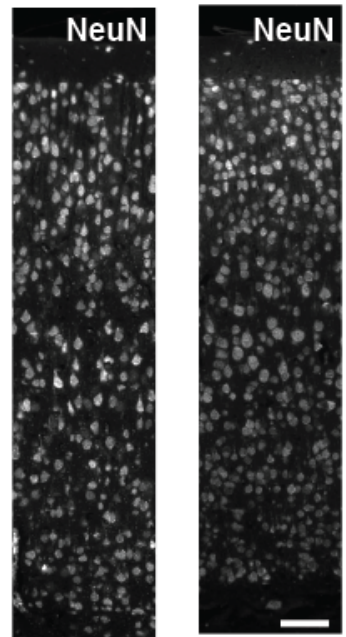

NEX-Cre; Nedd4-1 $1^{\text {fl/t. }}$ Nedd4-2 $2^{\mathrm{f} / \mathrm{f}}$

Figure 3-2. Reduced Thickness of Cortices in NEX-Cre;Nedd4$1^{f / f} ;$ Nedd4-2 $2^{f / f}$ Mouse Brains

(A) Anatomical studies on Nedd4-1/ff; $N$ edd4-2 $2^{\text {fff }}$ (left) and NEX-Cre;Nedd4$1^{f f f} ;$ Nedd4-2 $2^{\mathrm{fff}}$ (right) mouse brains at P16. $5 \mu \mathrm{m}$-thick brain paraffin sections were immunostained with an anti-NeuN antibody. Note that the thickness of the cortex was reduced in the NEX-Cre;Nedd4-1/ff; $N$ edd4 $42^{\text {fff }}$ brain. Scale bars, $500 \mu \mathrm{m}$. (B) High-magnification images of the cortices shown in $(A)$ revealed a reduction in thickness of cortex in the NEX-Cre;Nedd4-1/fif $N$ Nedd4$2^{f f f}$ brains (right). Scale bars, $50 \mu \mathrm{m}$. (C) Reduced thickness of the cortex in the NEX-Cre;Nedd4-1 $1^{f / f} ;$ Nedd $4-2^{f / f}$ brain $(1.157 \pm 0.0173 \mathrm{~mm}, \mathrm{~N}=11$ slices from 4 animals) as compared to the control Nedd4- ${ }^{f / f} ; N e d d 4-2^{\mathrm{fff}}$ brain (1.459 $\pm 0.0245 \mathrm{~mm}, \mathrm{~N}=8$ slices from 4 animals). ${ }^{* * *} p<0.0001$, unpaired $t$ test. (D) The number of neurons in a cortical region with the width of $100 \mu \mathrm{m}$. No significant difference in the number of neurons was observed between the two groups. Nedd4-1/ff; $N$ edd4-2 ${ }^{\mathrm{fff}}, 142 \pm 2, \mathrm{~N}=8$ slices from 4 animals; NEXCre;Nedd4- $1^{f / f} ; \mathrm{Nedd} 4-2^{\mathrm{fff}}, \quad 140 \pm 5, \mathrm{~N}=11$ slices from 4 animals. Data are expressed as mean \pm SEM. 
A

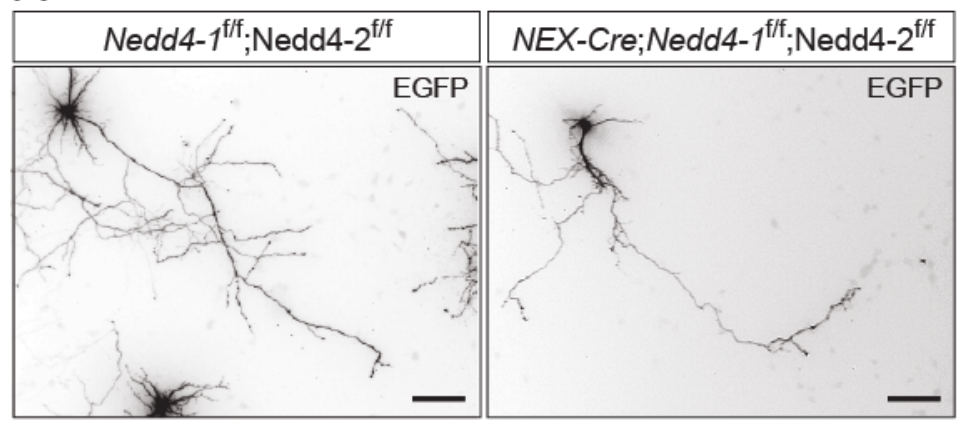

D

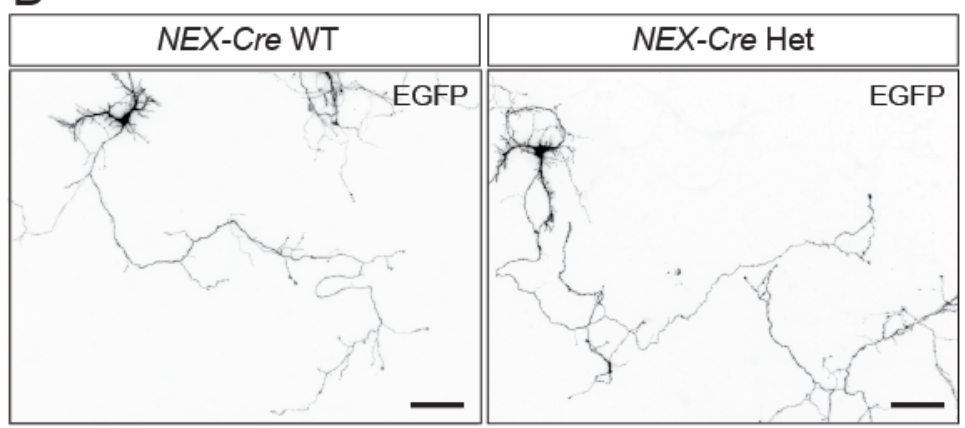

$B$

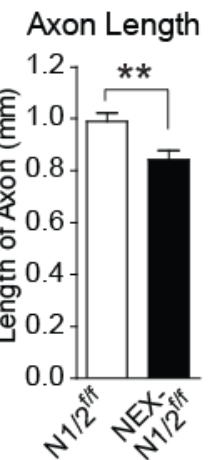

E Axon Length Axon Branches

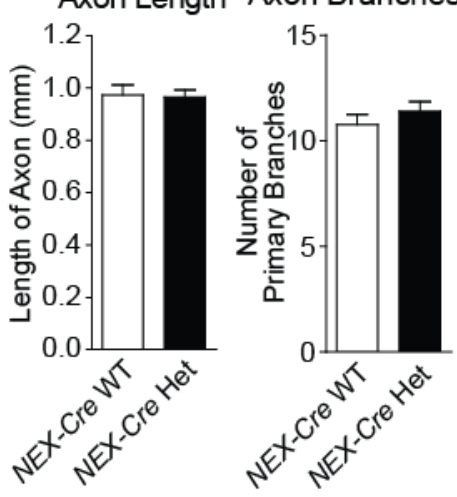

Figure 3-3. Nedd4-1 and Nedd4-2 Regulate Axon Growth in Mouse Hippocampal Neurons in vitro

(A) Representative images of control (left) and Nedd4-1 and Nedd4-2 double KO (right) cultured hippocampal neurons transfected with an EGFP expressing vector. Scale bars, $100 \mu \mathrm{m}$. (B) Reduced length of main axon shafts in Nedd4-1;Nedd4-2 double KO neurons. N1/2 $2^{\mathrm{ff}}, 0.99 \pm 0.033 \mathrm{~mm}$, $\mathrm{N}=92$; NEX-N1/2 ${ }^{\mathrm{f} / \mathrm{f}}, 0.84 \pm 0.035 \mathrm{~mm}, \mathrm{~N}=81 .{ }^{* *} p=0.0026$, unpaired $t$ test. $(C)$ Reduced number of primary axonal branches in Nedd4-1;Nedd4-2 double KO neurons. N1/2 $2^{\mathrm{fff}}, 11 \pm 0.5, \mathrm{~N}=92$; NEX-N1/2 $2^{\mathrm{fff}}, 8 \pm 0.4, \mathrm{~N}=81 .{ }^{* * *} p<0.0001$, unpaired $t$ test. $(D)$ Representative images of NEX-Cre wildtype (WT, left) and NEX-Cre heterozygote (Het, right) cultured hippocampal neurons transfected with an EGFP expressing vector. Scale bars, $100 \mu \mathrm{m}$. ( $E$ and $F$ ) No difference in length of main axon shafts $(E)$ and in number of primary axonal branches $(F)$ in NEX-Cre Het neurons as compared to NEX-Cre WT neurons are detectable, indicating that the phenotypic change observed in $(A-C)$ is not due to the NEX-Cre mutation itself. 
I further performed in utero electroporation to study the roles of Nedd41 and Nedd4-2 in axonal morphogenesis in vivo. At embryonic stage 15.5 (E15.5), plasmid DNA was transfected to a subpopulation of neuronal progenitor cells, which generate mainly layer II/III cortical neurons that project axons to the contralateral cortex (callosal projections). The axonal morphology of neurons was visualized by overexpressing a membraneanchored variant of the Venus yellow fluorescent protein (myrVenus), and Nedd4-1/Nedd4-2 were eliminated by co-expression of Cre in Nedd4$1^{f / f} ;$ Nedd4- $2^{f / f}$ embryos. The brains were fixed at P21, when the axons of upper layer cortical neurons have reached the contralateral side and branched extensively into the somatosensory cortex. Most of the myrVenusexpressing neurons migrated properly to the upper cortical layers of the ipsilateral side independently of the Cre expression (Figure 3-4A), indicating that Nedd4-1 and Nedd4-2 are dispensable for neuronal migration (see also Figures 3-2 $A$ and $B$ ). Both control and Nedd4-1;Nedd4-2 KO neurons projected axons to the contralateral side of the cortex, but the extent of axon invasion as assessed by myrVenus signal intensity was lower with Nedd41; Nedd4-2 KO neurons (Figures 3-4 $A$ and $B$ ). Callosal axons branched specifically in layers II/III and V on the contralateral side (Figure 3-4B) Interestingly, we observed a significant reduction of axonal branching in Creexpressing Nedd4-1;Nedd4-2 KO neurons (Figures 3-4 C and D). Taken together, these in vitro and in vivo results indicate that Nedd4 family E3 ligases, Nedd4-1 and Nedd4-2, play an evolutionarily conserved role in the regulation of axonal morphogenesis. 

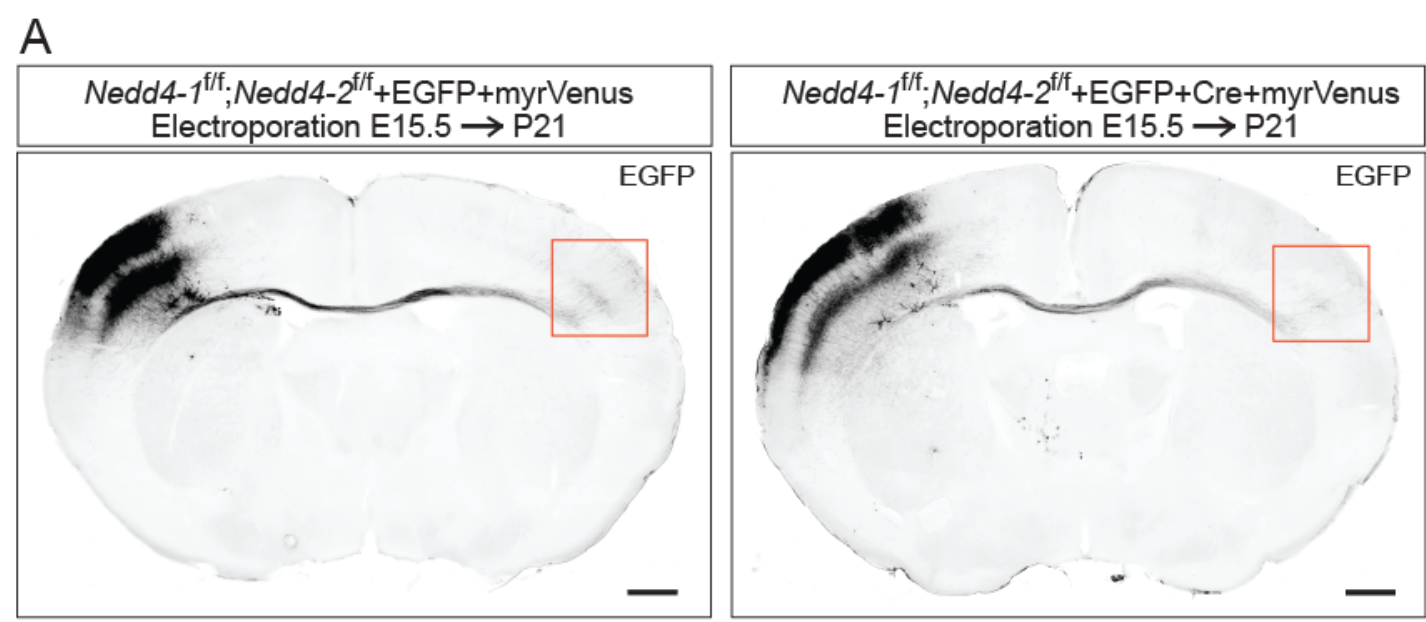

B
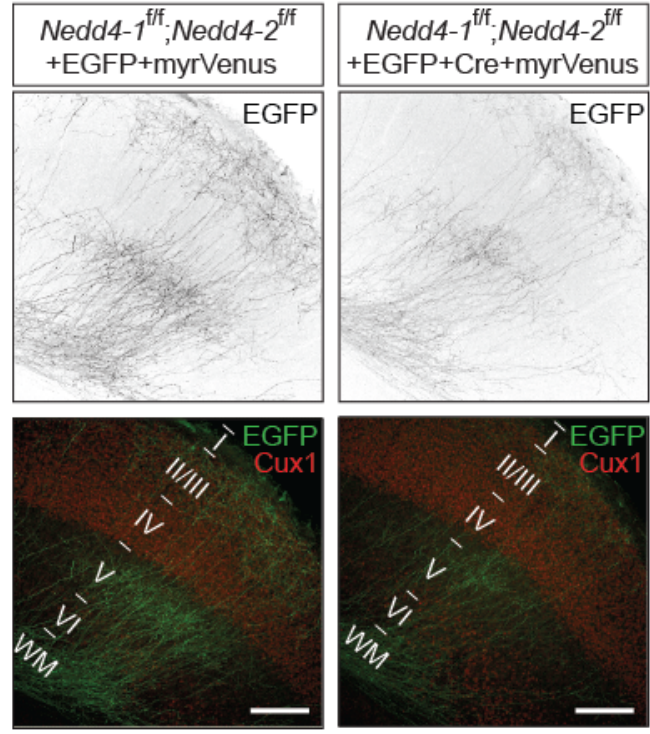

C
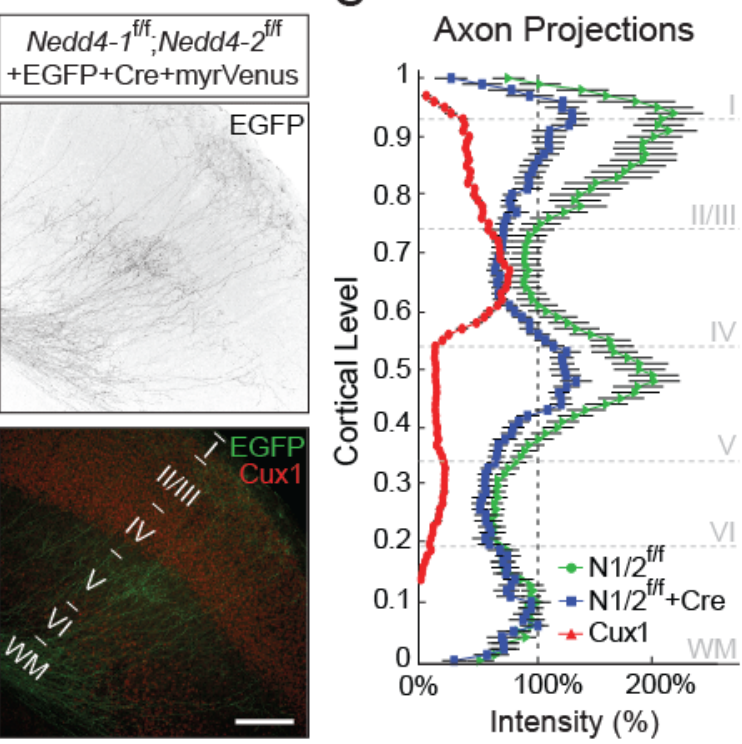

Figure 3-4. Nedd4-1 and Nedd4-2 Regulate Axon Growth in Mouse Cortical Neurons in vivo

(A) Nedd4-1 ${ }^{\mathrm{fff}} ; \mathrm{Nedd} 4-2^{\mathrm{fff}}$ embryos were co-transfected with pFUGW (EGFP) and pCX-myrVenus (left), or pFUGWiCre (EGFP + Cre) and pCX-myrVenus (right) expression vectors using in utero electroporation. Scale bars, $500 \mu \mathrm{m}$. $(B)$ High-magnification images of regions in red boxes in $(A)$. Immunostaining for Cux1 was used to label layer II-IV neurons. Scale bars, $200 \mu \mathrm{m}$. (C) Quantification of the signal intensity from the cortical surface (cortical level 1) to the bottom of the white matter (WM) (cortical level 0 ) for axon projections at the contralateral side [see $(A)$ and $(B)$ ]. The maximum signal in the white matter was set to $100 \%$ intensity. $\mathrm{N} 1 / 2^{\mathrm{f} / \mathrm{f}}, \mathrm{N}=19$ from 8 animals; NEX-N $1 / 2^{\mathrm{ft/f}}$, $\mathrm{N}=11$ from 5 animals. $(D)$ Significant reduction in maximum intensity of axonal projections of Nedd4-1;Nedd4-2 double KO neurons in the upper layer and lower layer of the cortex [see $(A-C)]$. Upper layer (cortical level 0.71): $\quad \mathrm{N} 1 / 2^{\mathrm{fff}}, \quad 269.8 \pm 27.72 \% ; \quad \mathrm{N} 1 / 2^{\mathrm{fff}}+\mathrm{Cre}, \quad 161.7 \% \pm 14.20 \% ; \quad{ }^{* *} p=0.0019$, unpaired $t$ test with Welch's correction. Lower layer (cortical level 0.25-0.69): $\mathrm{N} 1 / 2^{\mathrm{fff}}, 222.9 \% \pm 20.81 \% ; \mathrm{N} 1 / 2^{\mathrm{fff}}+\mathrm{Cre}, 149.1 \% \pm 8.64 \% ;{ }^{* *} p=0.0033$, unpaired $t$ 
test with Welch's correction. Data are expressed as mean $\pm \mathrm{SEM} . \mathrm{N} 1 / 2^{\mathrm{f} / \mathrm{f}}$, Nedd4- ${ }^{\mathrm{f} / \mathrm{f}} ;$ Nedd4-2/f. NEX-N1/2/f/ . NEX-Cre;Nedd4-1/f/f;Nedd4-2/f.

\subsubsection{PTEN is not Targeted by Nedd4-1 or Nedd4-2 for Proteosomal Degradation}

Given that PTEN was reported to be targeted by Nedd4-1 or Nedd4-2 for proteasomal degradation in various tissues and organisms (Wang et al., 2007; Drinjakovic et al., 2010; Yim et al., 2009; Christie et al., 2012), I next examined if PTEN is a substrate of Nedd4 family E3 ligases in the process of axon growth regulation. I studied the levels of PTEN by Western blotting of lysates prepared from developing neurons using an antibody to endogenous PTEN (see Figure 3-8A for the antibody specificity). Cre was expressed in Nedd4-1 ${ }^{\mathrm{f} / \mathrm{f}} ;$ Nedd4-2/f cultured hippocampal neurons using lentiviral infection in order to eliminate Nedd4-1 and Nedd4-2 from all cell types in the culture dish, including a small fraction of glial cells. Despite the fact that mammalian Nedd4-1 and Nedd4-2 are important for axon growth (Figures 3-3 and 3-4), I did not detect any concomitant upregulation of PTEN in cultured neurons at DIV8 upon deletion of Nedd4-1/Nedd4-2 (Figures 3-5 $A$ and $B$, left panels). Rather, the PTEN protein levels were significantly reduced in Nedd41;Nedd4-2 KO neurons at DIV15 (Figures 3-5 $A$ and $B$, right panels), strongly arguing against that PTEN is targeted by Nedd4-1 or Nedd4-2 for proteosomal degradation. Overexpression of exogenous Cre does not affect Nedd4-1 and PTEN levels in wild-type neurons (Figures 3-5 $C$ and $D$ ), indicating that the results $I$ found in Figures 3-5 $A$ and $B$ are specific outcomes of Nedd4-1 and Nedd4-2 deletion in neurons. Similar results that no upregulation of the PTEN level upon deletion of Nedd4-1 and Nedd4-2 were also obtained from P7 (Figures 3-5 E and F) and 5-week-old (Figures 3$5 G$ and $H$ ) NEX-N1/2/f brains, where Nedd4-1 and Nedd4-2 were deleted in postmitotic glutamatergic neurons. Taken together, these in vitro and in vivo data indicate that Nedd4-1 and Nedd4-2 are dispensable for proteasomal degradation of PTEN in developing mammalian neurons. 
A

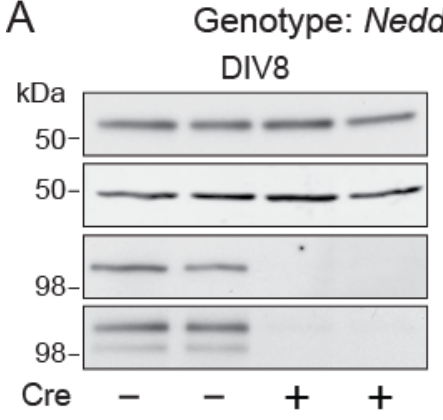

C Genotype: wildtype

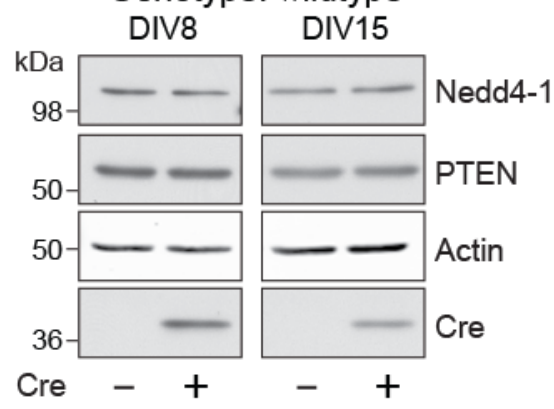

E P7 Mouse Brain Lysate

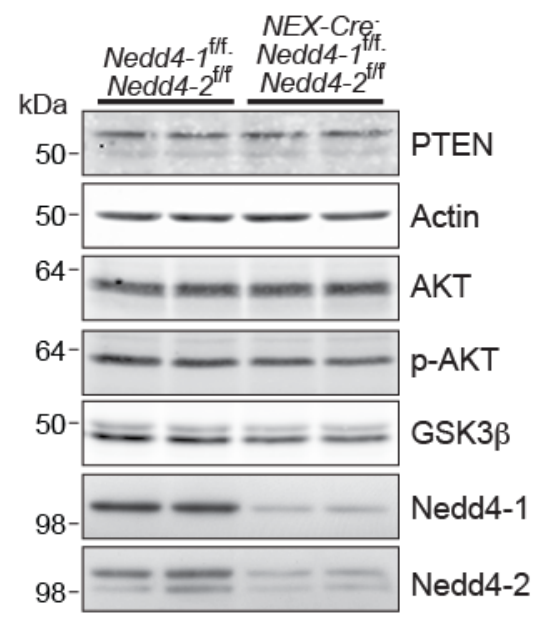

B

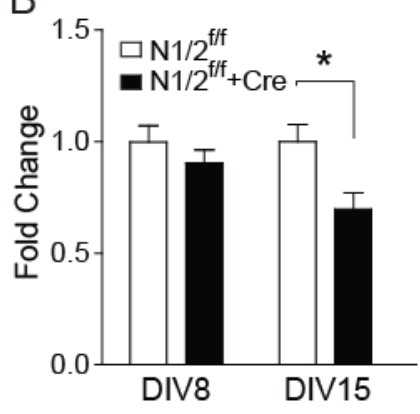

DIV8
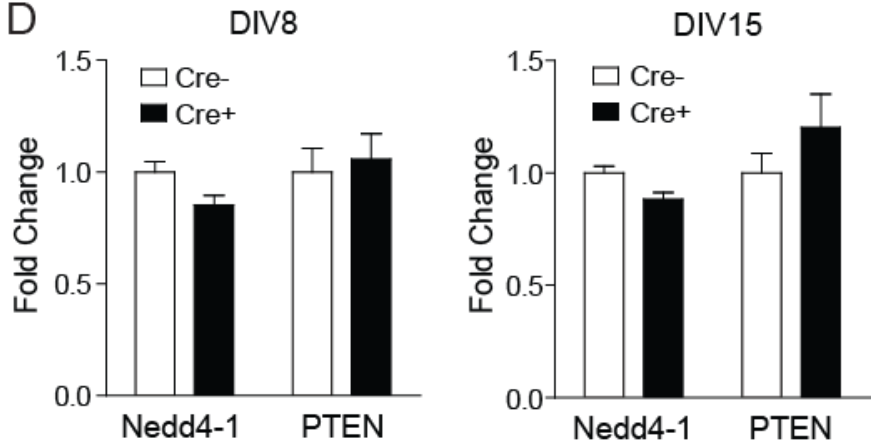

F

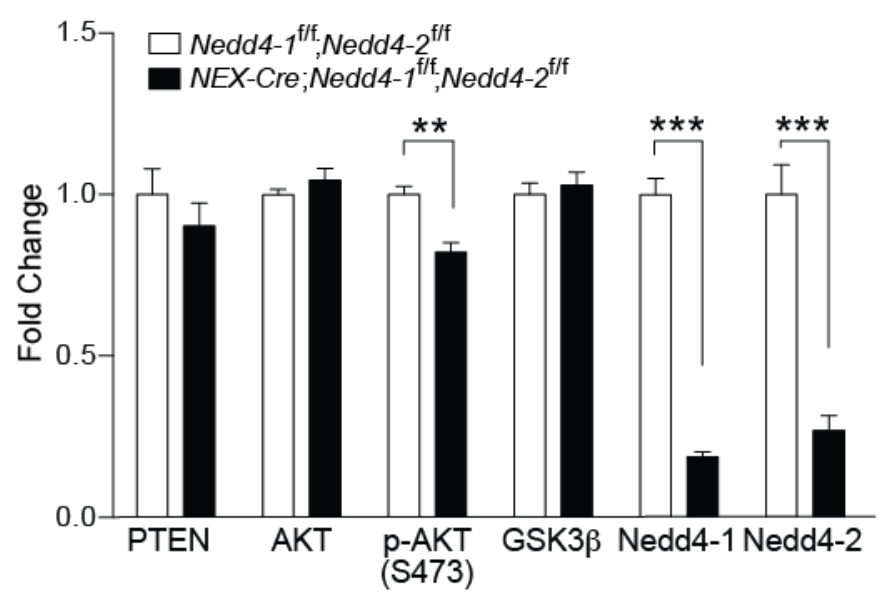

G 5-Week-Old Mouse Brain Lysate $H$
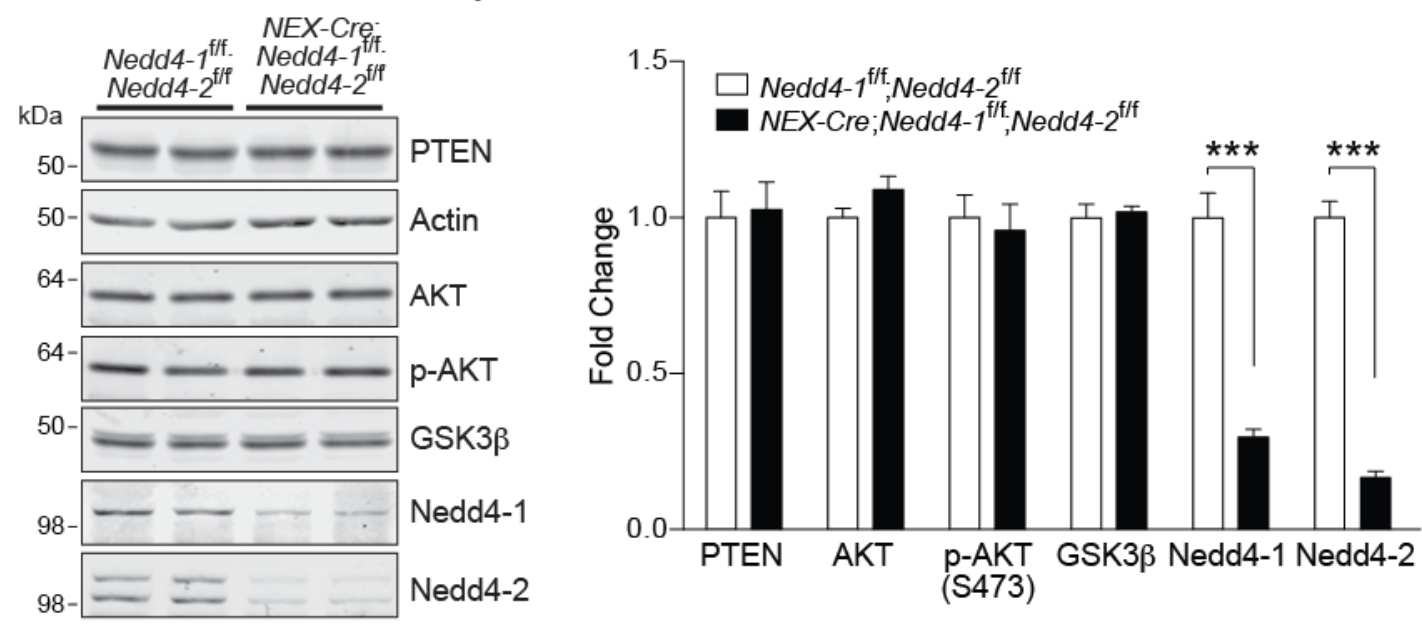
Figure 3-5. Normal Expression of PTEN in Neurons upon Deletion of Nedd4-1 and Nedd4-2 in vitro and in vivo

(A) Representative Western blotting results showing that upon Nedd4-1 and Nedd4-2 deletion in cultured neurons, PTEN levels were unaltered at DIV8 and downregulated at DIV15. Primary hippocampal neurons were prepared from Nedd4- ${ }^{f / f} ; N e d d 4-2^{f / f}$ mice and Cre was expressed using lentiviral infection. (B) Quantification of PTEN levels by Western blotting [see $(A)$ ]. Signals for PTEN were normalized to signals for actin. ${ }^{*} p=0.0471, N=3$ per condition, unpaired $t$ test. (C) Representative Western blotting results showing no difference in PTEN and Nedd4-1 levels when Cre was expressed in wild type hippocampal neurons using lentiviral infection, indicating that overexpression of exogenous Cre does not affect Nedd4-1 and PTEN levels in neurons. Neurons were harvested on DIV8 and DIV15. (D) Quantification results of Western blotting [see $(B)$ ] for PTEN and Nedd4-1 levels at DIV8 (left) and at DIV 15 (right). No statistical differences were observed between groups. $\mathrm{N}=3$ per group. Data are expressed as mean \pm SEM. ( $E$ and $G$ ) Representative Western blotting results showing levels of PTEN, AKT, phospho-AKT (p-AKT, Ser473), and GSK3 $\beta$ in brain lysates prepared from P7 mice $(E)$ and 5-week-old mice $(G)$ with the indicated genotypes. $(F$ and $H)$ Quantification of Western blotting results in $(E)$ [see $(F)$ ] and $(G)$ [see $(H)$ ]. Signals from the candidate proteins were normalized to the loading control. Phospho-AKT levels were normalized to total AKT levels and showed a slight

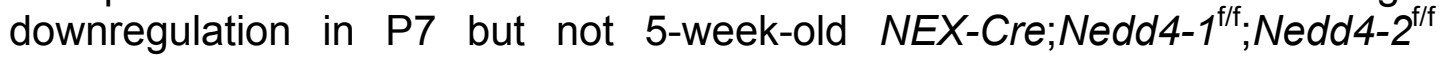
brains. PTEN, AKT, and GSK3 $\beta$ showed no difference in both P7 and 5week-old NEX-Cre;Nedd4-1/f/Nedd4-2/f brains as compared to controls. The residual expression of Nedd4-1 and Nedd4-2 in NEX-Cre;Nedd4- $1^{\mathrm{f} / \mathrm{f}}$;Nedd4$2^{\mathrm{f} / \mathrm{f}}$ brains is likely from glial cells and inhibitory neurons. Given that PTEN levels and phosphatase activity were not affected (Figures 3-6 $C$ and $D$ ), the decreased phospho-AKT (Ser473) levels in the P7 NEX-Cre;Nedd4$1^{\mathrm{f} / \mathrm{f}} ;$ Nedd4-2 ${ }^{\mathrm{f} / \mathrm{f}}$ brain are likely due to misregulation of other substrates of Nedd4 family E3 ligases [e.g. growth factor receptors or phosphatases for phospho-AKT (Ser473)]. ${ }^{* *} p=0.002 ;{ }^{* * *} p<0.001$, unpaired $t$ test. $\mathrm{N}=5$ per group. Data are expressed as mean \pm SEM.

\subsubsection{PTEN Neither Poly- Nor Mono-ubiquitinated by Nedd4-1/Nedd4-2 in Developing Mammalian Neurons}

A recent report indicated that Nedd4-1 conjugates K63-linked polyubiquitin chains to PTEN and thereby inhibits the phosphatase activity of PTEN without affecting its stability (Guo et al., 2012). To further confirm that PTEN is not polyubiquitinated by Nedd4-1 or Nedd4-2 in developing neurons, endogenous PTEN was immunoprecipitated from lysates of cortices prepared from $\mathrm{P} 7$ control and NEX-N1/2/f mice. The ubiquitination level of 
PTEN was then detected by Western blotting using anti-ubiquitin antibodies. In order to avoid ubiquitination signals from co-immunoprecipitated PTENbinding proteins, I performed immunoprecipitation of proteins under denaturing conditions. No reduction in the PTEN polyubiquitination levels was observed in NEX-N1/2 f/f brains (Figures 3-6 $A$ and $B$ ), further supporting the notion that PTEN is not polyubiquitinated by Nedd4-1 or Nedd4-2 in developing neurons. Next, I assessed the phosphatase activity of immunoprecipitated PTEN using an in vitro phosphatase assay. I titrated different amounts of the anti-PTEN antibody for immunoprecipitation (Figure 3-6C), and measured the activity of PTEN immunoprecipitated from control or NEX-N $1 / 2^{\mathrm{f} / \mathrm{f}}$ mouse brains at the non-plateau phase of the assay where the readout of PTEN activity in the immunoprecipitates was not saturated. I found that the phosphatase activity of PTEN was unaltered in NEX-N1/2 f/f brains (Figure 3-6D).

Monoubiquitination by Nedd4-1 was reported to cause nuclear import of PTEN in mouse embryonic fibroblasts (Trotman et al., 2007). In view of this, I studied the subcellular distribution of PTEN in control and NEX-N1/2 f/f neurons to examine if Nedd4-1 or Nedd4-2 regulates the compartmentalization of PTEN. I performed immunostaining of PTEN on cultured neurons at DIV7 using a specific antibody to PTEN (Figure 3-7A). In mouse hippocampal neurons, PTEN immunoreactivity was mostly confined to the cell body and proximal neurites, and very weak in axonal growth cones for both control and NEX-N1/2 f/f neurons (Figures 3-7 $B$ and $C$ ). The latter finding is strikingly different from data obtained in Xenopus laevis retinal ganglion cells (Drinjakovic et al., 2010). Importantly, PTEN was not noticeably enriched in nuclei, and the ratio of nuclear vs. total cellular PTEN was not altered in NEX-N1/2/f neurons (Figures 3-7 $D$ and $E$ ), indicating that the subcellular localization of PTEN in developing murine neurons is not regulated by Nedd4-1- or Nedd4-2-mediated monoubiquitination. Taken all together, these data indicate that Nedd4 family E3 ligases do not ubiquitinate PTEN, control PTEN localization, or affect the phosphatase activity of PTEN in developing mammalian neurons. 
A

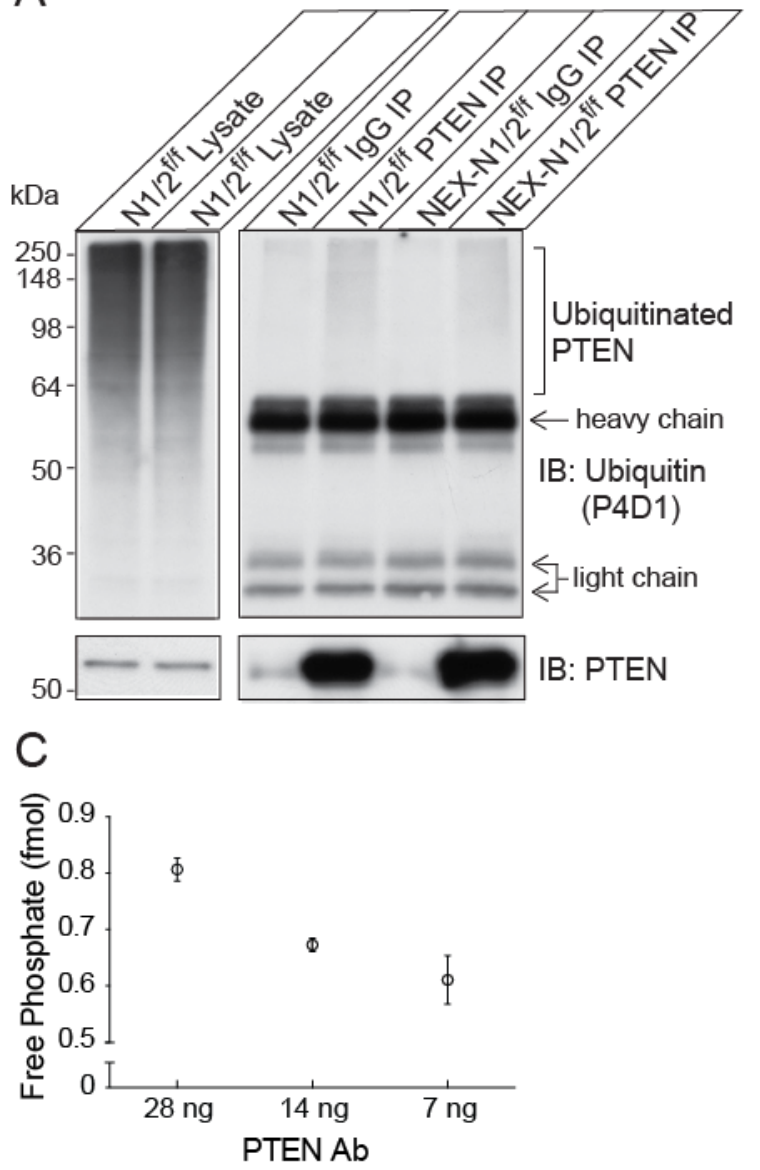

B
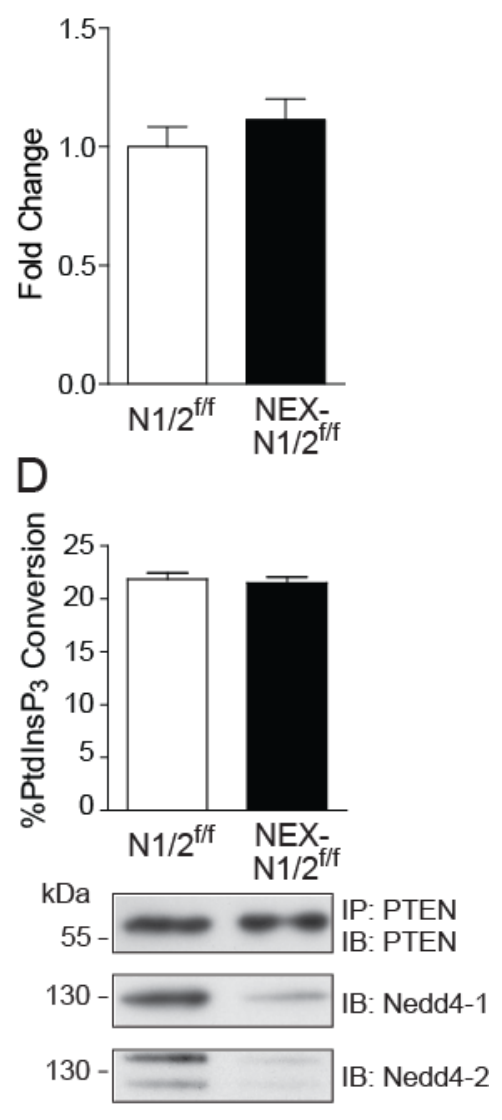

Figure 3-6. Normal Ubiquitination and Phosphatase Activity of Endogenous PTEN in Neurons upon Deletion of Nedd4-1 and Nedd4-2

(A) No reduction of PTEN polyubiquitination levels in NEX-N1/2 ${ }^{\mathrm{f} / \mathrm{f}}$ mouse brains. (B) Quantification of PTEN polyubiquitination levels [see $(A)$ ]. The smear pattern (over $60 \mathrm{kD}$ ) was quantified and normalized to signals of immunoprecipitated PTEN. $\mathrm{N}=3$ per genotype, unpaired $t$ test. (C) Endogenous PTEN was immunoprecipitated from P7 mouse brain lysates with the indicated amount of anti-PTEN antibodies, and subjected to the PTEN phosphatase activity assay. Signals from the 'no PTEN' negative control were subtracted from all groups. Note that the readout of the assay was dependent on the PTEN amount. (D) Endogenous PTEN was immunoprecipitated with $14 \mathrm{ng}$ of the anti-PTEN antibody [see $(C)$ ]. Normal PTEN phosphatase activity in P7 NEX-N1/2/f brains as compared to N1/2 f/f brains. Levels of immunoprecipitated PTEN (top blot) and genotypes of animals (middle and bottom blots) were confirmed by Western blotting. $p=0.6859, N=3$ per genotype, unpaired $t$ test. Data are expressed as mean \pm SEM. N1/2 f/f,$\quad N e d d 4-1^{\mathrm{fff}} ; \mathrm{Nedd4} 2^{\mathrm{f} / \mathrm{f}}$. NEX-N1/2 ${ }^{\mathrm{f} / \mathrm{f}}$, NEX-Cre;Nedd4$1^{\mathrm{f} / \mathrm{f}} ;$ Nedd4- $2^{\mathrm{f} / \mathrm{f}}$. IB, immunoblotting. IP, immunoprecipitation. 


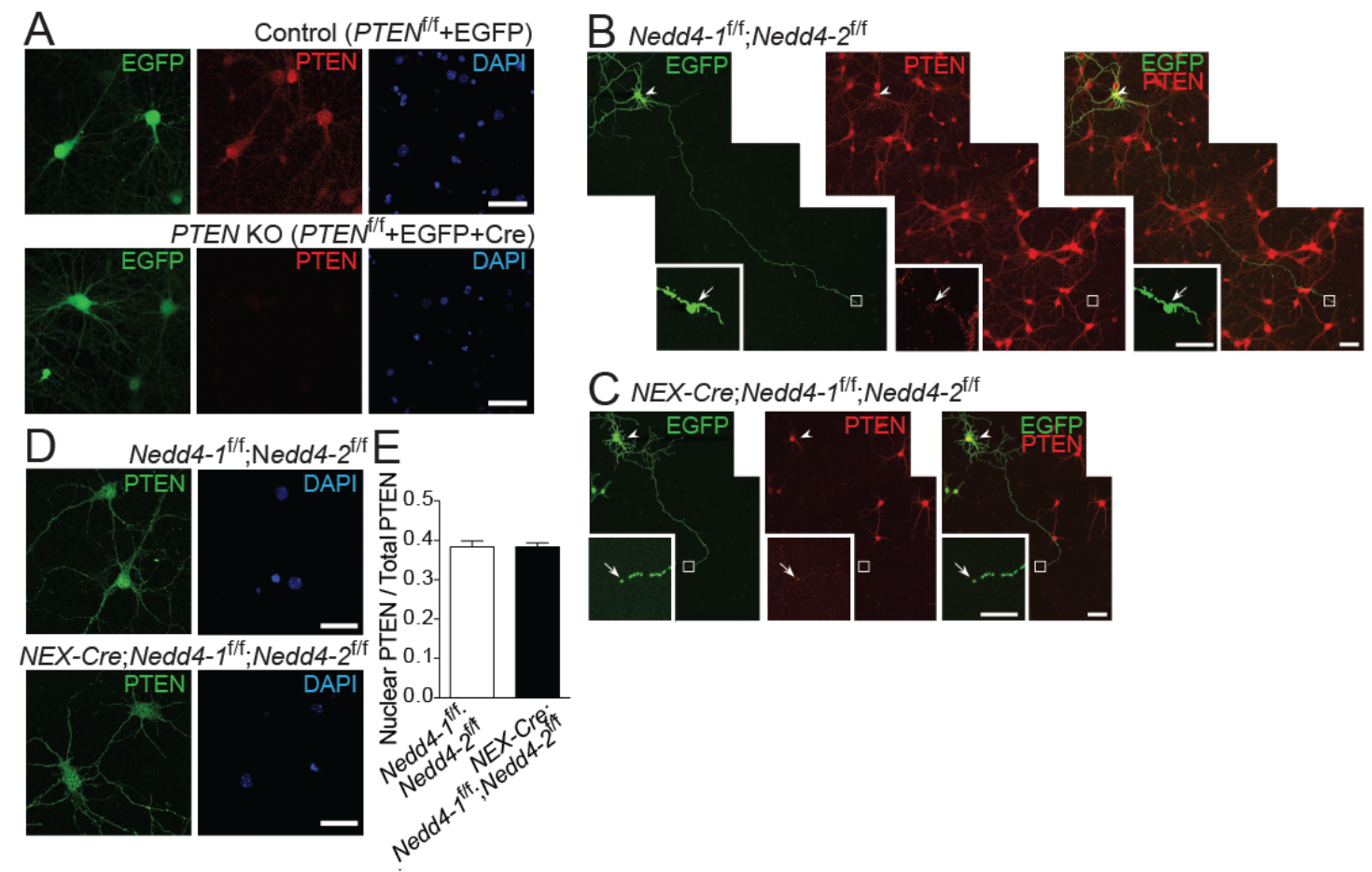

Figure 3-7. Normal Localization of PTEN upon Deletion of Nedd4-1 and Nedd4-2

(A) Specificity of the anti-PTEN antibody (clone D4.3; Cell Signaling) in immunostaining. Scale bars, $50 \mu \mathrm{m}$. ( $B$ and $C$ ) Overview of the subcellular localization of PTEN in hippocampal neurons prepared from Nedd4$1^{f / f} ;$ Nedd4- $2^{\mathrm{fff}}$ mice $(B)$, and NEX-Cre;Nedd4 $-1^{\mathrm{fff} ;} ; \mathrm{Nedd} 4-2^{\mathrm{fff}}$ mice $(C)$. Neurons were transfected with an EGFP expression vector at DIV1, fixed at DIV7, and immunostained for endogenous PTEN. High-magnification images of regions in white boxes are shown in the lower left panels. The soma and the axonal growth cone of the EGFP-expressing neuron are indicated by arrowheads and arrows, respectively. Note that PTEN signals at the axonal growth cone were very weak as compared to those in the soma and proximal neurites. Scale bars, $50 \mu \mathrm{m}$ for low-magnification images, and $10 \mu \mathrm{m}$ for highmagnification images. $(D)$ Representative images for control (Nedd4-

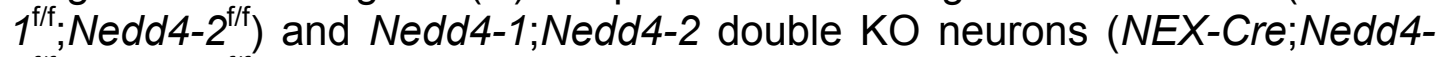
$1^{\mathrm{fff}} ;$ Nedd4-2 $2^{\mathrm{fff}}$ ) immunostained for endogenous PTEN. Scale bars, $50 \mu \mathrm{m}$. (E) Quantification demonstrated no significant difference between the two groups $[($ see $(D)]$ in the ratio of nuclear PTEN vs. total PTEN in the soma. $p=0.991$, unpaired $t$ test with Welch's correction. Nedd4-1 ${ }^{\mathrm{fff}} ; \mathrm{Nedd4}-2^{\mathrm{fff}}, \mathrm{N}=83 ; N E X-$ Cre;Nedd4-1 $1^{\mathrm{fff}} ; \mathrm{Nedd} 4-2^{\mathrm{fff}}, \mathrm{N}=78$. Data are expressed as mean \pm SEM. 


\subsubsection{PTEN Acts as a Negative Regulator of Nedd4-1 Expression at the Translational Level}

Although I excluded the possibility that PTEN acts as a downstream target of Nedd4-1/Nedd4-2 in the regulation of axon growth, several lines of evidence indicate an inverse correlation between the expression levels of Nedd4-1 and PTEN (Ahn et al., 2008; Amodio et al., 2010; Chung et al., 2011; Kwak et al., 2010; Wang et al., 2007). These findings are compatible with the notion that PTEN may act as an upstream negative regulator of Nedd4-1 or Nedd4-2 expression. However, others reported a positive correlation between Nedd4-1 and PTEN expression (Yang et al., 2008b). To study if and how PTEN regulates the levels of Nedd4 family E3 ligases in developing neurons, I examined expression levels of Nedd4 family E3 ligases in control and PTEN KO neurons. Of note, Nedd4-1 protein levels were significantly upregulated in the absence of PTEN while Nedd4-2 levels were unaltered, indicating that PTEN acts as an upstream negative regulator of Nedd4-1 expression in developing neurons (Figure 3-8A, left two lanes and Figure 3-8B).

Given that PTEN is the prime negative regulator of PI3K-regulated intracellular signaling, I further investigated the possible involvement of signaling molecules of the PI3K/PTEN cascade in the regulation of Nedd4-1 expression. Among those downstream molecules, I focused on mTORC1 (serine/threonine protein kinase mammalian target of rapamycin complex 1), because it was reported to control dendrite growth (Jaworski et al., 2005). To test if mTORC1 is involved in the upregulation of Nedd4-1 expression upon PTEN deletion, I treated control and PTEN KO neurons with rapamycin, an inhibitor for mTORC1. Strikingly, the upregulation of Nedd4-1 expression in PTEN KO neurons was reverted by rapamycin treatment (20 nM for $24 \mathrm{~h}$; Figure 3-8A, right two lanes and Figure 3-8B), indicating that the mTORC1 activity is required for the PTEN-dependent regulation of Nedd4-1 expression. Importantly, PTEN deletion did not affect Nedd4-1 mRNA expression while neurofilament $H(N F H)$ mRNA, which was used as a 
positive control, showed a significant upregulation upon PTEN loss (Figure 3$8 C$ ). These results indicate that the PTEN-mTORC1 pathway functions upstream of Nedd4-1 to negatively regulate Nedd4-1 protein expression at the posttranscriptional level.

I further investigated whether PTEN regulates Nedd4-1 mRNA translation or affects Nedd4-1 protein stability. In collaboration with Rossella Luca and Dr. Claudia Bagni (Leuven, Belgium), actively translating polysomes and silent mRNPs were isolated from cultured control and PTEN KO neurons using a continuous sucrose gradient. We found that Nedd4-1 mRNA was more strongly associated with the polysomal fraction in PTEN KO neurons, as compared to control cells. Similar findings were obtained for Rp/13a mRNA, as shown previously (Thoreen et al., 2012), whereas the polysomal association of Nedd4-2 and GAPDH mRNAs was not changed in PTEN KO neurons (Figures 3-8 $D$ and $E$ ). Because Nedd4-1 protein stability is not changed in PTEN KO neurons (Figure 3-8F), increased Nedd4-1 mRNA translation is the likely cause for the observed increase in Nedd4-1 protein levels (Figures 3-8 $A$ and $B$ ). These results indicate that the PTENmTORC1 pathway functions upstream of Nedd4-1 to negatively regulate Nedd4-1 mRNA translation in developing mammalian neurons. 

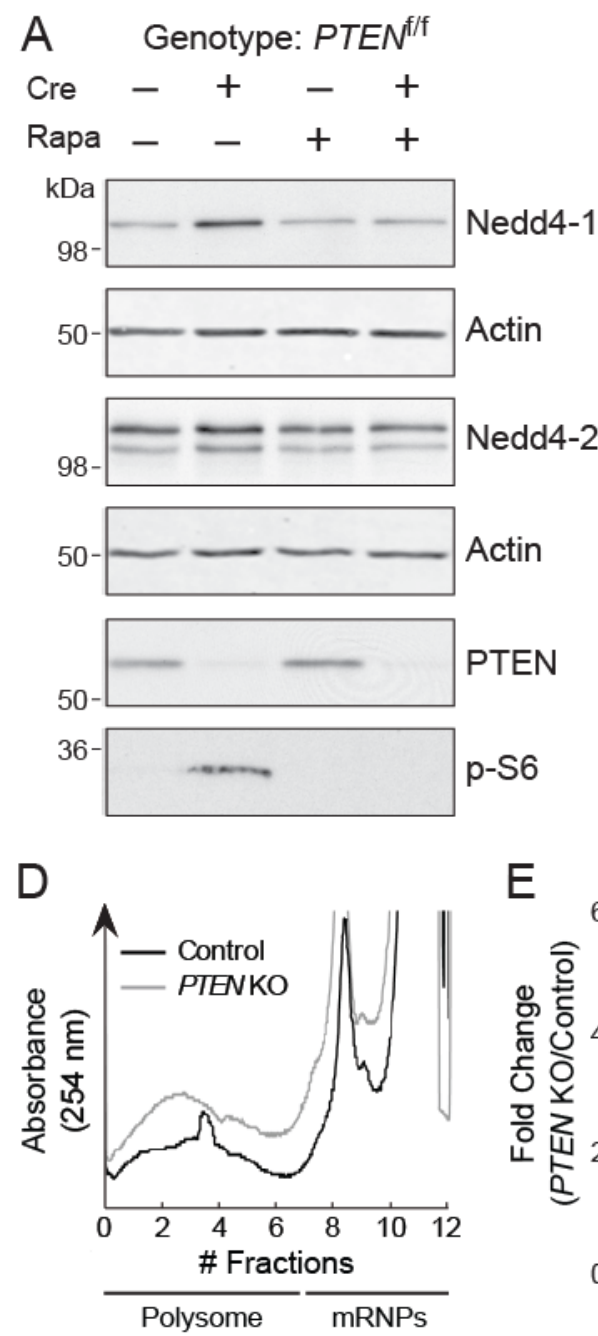
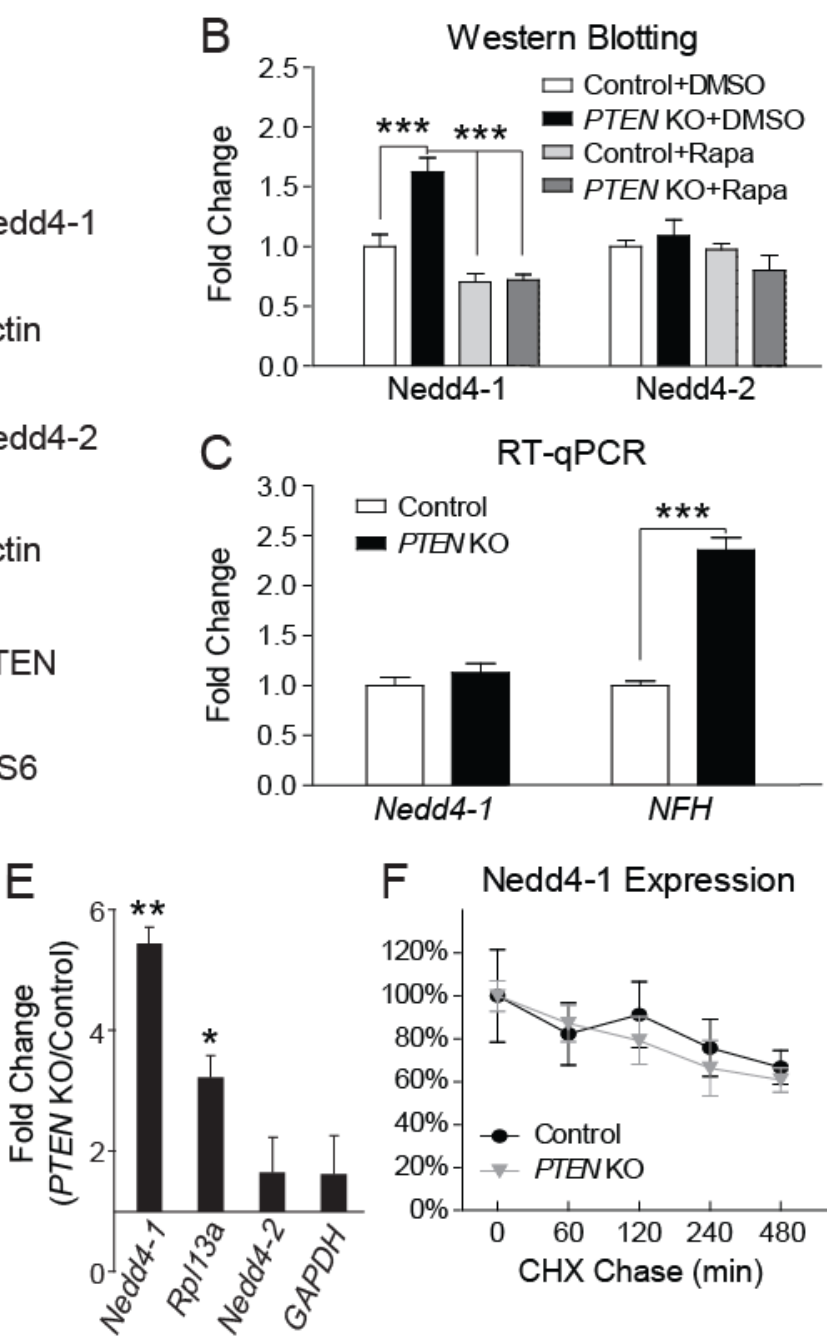

Figure 3-8. PTEN Negatively Regulates Nedd4-1 Expression at the Translational Level

(A) Representative Western blotting results showing that upon deletion of PTEN, Nedd4-1 but not Nedd4-2 protein levels were upregulated (left two lanes) at DIV8. This upregulation was abolished by rapamycin treatment (right two lanes). Primary hippocampal neurons were prepared from PTEN $N^{\text {f/f }}$ mice and Cre was expressed using lentiviral infection. Reduction in phosphoS6 (p-S6) levels was used as a positive control for rapamycin treatment. $(B)$ Quantification of Western blotting results (see $A$ ). ${ }^{* * *} p<0.001$, one-way ANOVA and Bonferroni's post hoc test, $\mathrm{N}=5$ per condition. (C) Real-time quantitative PCR results showing that the Nedd4-1 mRNA level in mouse hippocampal neurons is not altered in the absence of PTEN. Neurofilament $H$ $(N F H)$ mRNA was used as a positive control for PTEN deletion. ${ }^{* * *} p<0.0001$ unpaired $t$ test, $\mathrm{N}=4$ per condition. $(D)$ Representative polysome-messenger ribonucleoprotein (mRNPs) distribution on a sucrose gradient for cultured control and PTEN KO neurons. Fractions 1-7 correspond to polysomeassociated (translating) mRNAs. (E) Quantification of the relative translational efficiency of Nedd4-1, Rp/13a, Nedd4-2, and GAPDH mRNAs reported as the ratio of polysome-associated mRNAs over total mRNAs. 
${ }^{* *} p=0.004 ;{ }^{*} p=0.018$ unparied $t$ test, $\mathrm{N}=3$. Rp/13a was used as a positive control (Thoreen et al., 2012) while GAPDH was a negative control. $(F)$ Neurons (DIV8) were incubated with cycloheximide (CHX, $50 \mu \mathrm{g} / \mathrm{ml})$ for different duration as indicated, and the Nedd4-1 level was assessed by Western blotting. The remaining Nedd4-1 level over time showed no difference between the two groups, indicating that the stability of Nedd4-1 protein is not affected in PTEN KO neurons. Data are expressed as mean \pm SEM.

\subsubsection{Nedd4-1 is a Major Target of mTORC1 Signaling in Neurite Development}

To investigate the physiological importance of PTEN-regulated Nedd4-1 expression in developing neurons, I studied the impact of genetic elimination of Nedd4-1 on neurite development in the PTEN KO background in cultured hippocampal neurons (Figures 3-9 $A-E$ ). Consistent with a previous report (Kwon et al., 2006), PTEN KO led to hypertrophy of neurites (Figures 3-9 A and $B$ ). This phenotypic change is opposite to the one seen upon Nedd4-1 KO. Strikingly, additional KO of Nedd4-1 in PTEN KO neurons partially rescued the neurite hypertrophy phenotype (Figures 3-9 $B-E$ ), indicating that Nedd4-1 is a prominent regulator of neurite growth downstream of PTENdependent signaling pathways.

To study whether mTORC1 is involved in the PTEN- and Nedd4-1dependent regulation of neurite growth, I applied rapamycin to cultured control $\left(N e d d 4-1^{f / f}\right)$ and Nedd4-1 conditional KO (NEX-Cre;Nedd4-1 ${ }^{\text {f/f; }}$;NEX$\mathrm{N} 1^{f / f}$ ) neurons and quantified the neurite complexity (Figures 3-9 F-K). Consistent with previous findings (Jaworski et al., 2005), rapamycin treatment reduced the complexity of neurites in control neurons (Figures 3-9 $G, J$, and $K$ ). In NEX-N1/ff neurons, however, the effect of rapamycin was not significant (Figures 3-9 H-K), indicating that mTORC1 functions upstream of Nedd4-1 and plays a role in the regulation of Nedd4-1 expression to promote neurite growth. These results, together with my biochemical data (Figures 38 ), indicate that Nedd4-1 is a major downstream effector of the PI3K/PTENmTORC1 pathway in the regulation of neurite development. 

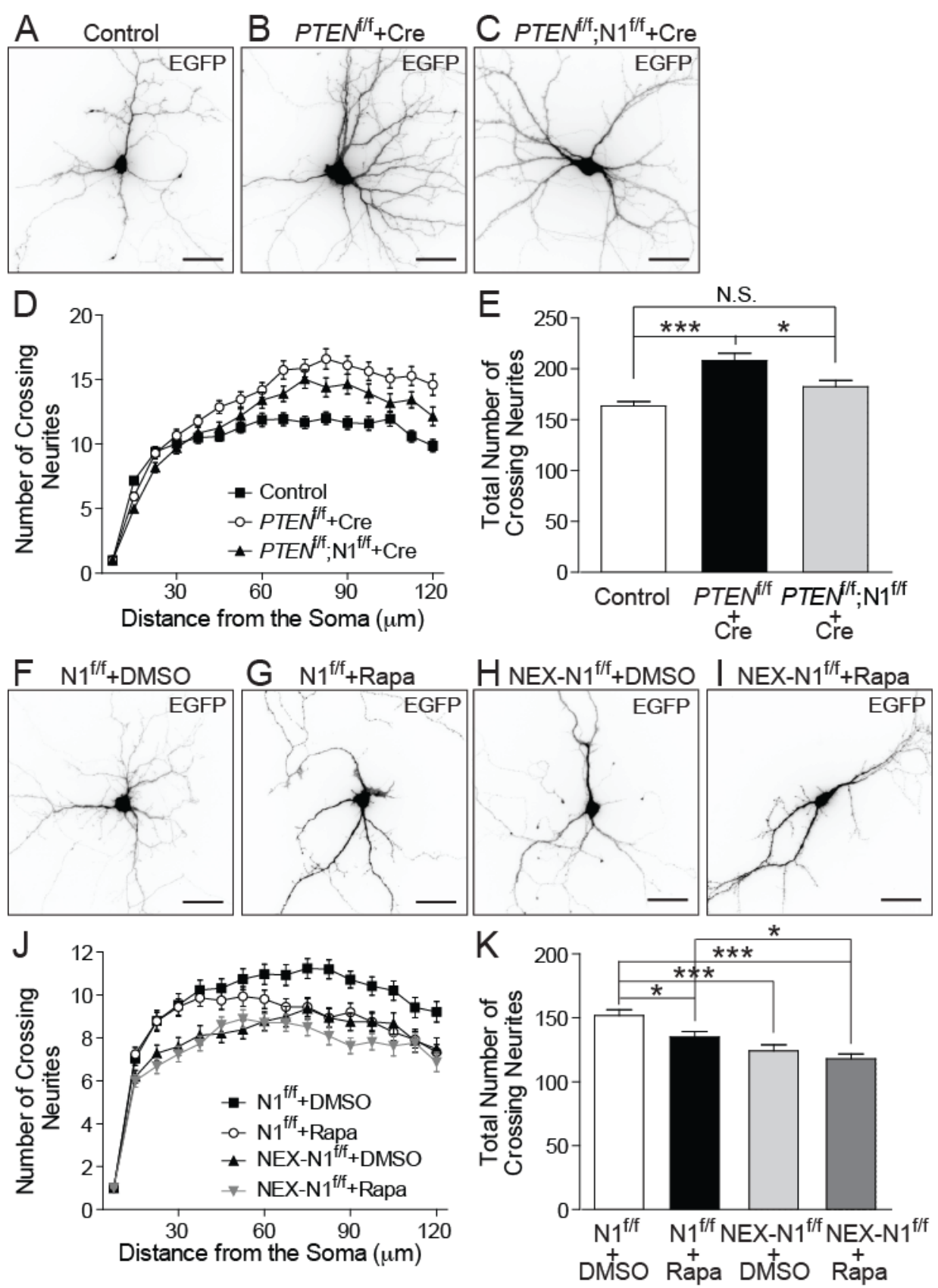

Figure 3-9. Nedd4-1 Has a Prominent Role in Neurite Morphogenesis and is Dependent upon mTORC1 Activity

$(A-C)$ Representative images of a control neuron $(A)$, a PTEN KO neuron $(B)$, and a PTEN;Nedd4-1 double KO neuron (C). Scale bars, $50 \mu \mathrm{m}$. (D) Sholl analyses for the three groups in $(A-C)$. Note that KO of Nedd4-1 partially rescues the hypertrophy of neurite structure seen in PTEN KO neurons. (E) Statistical analysis of the total number of crossing neurites obtained in the Sholl analysis shown in $(D)$. Data were analyzed by one-way ANOVA and Tukey's post hoc test ( $N>60$ neurons per group). N.S., not significant. $(F-I)$ Representative images of a control neuron treated with DMSO $(F)$, a control neuron treated with rapamycin $(G)$, a Nedd4-1 KO neuron treated with 
DMSO $(H)$, and a Nedd4-1 KO neuron treated with rapamycin $(I)$. Scale bars, $50 \mu \mathrm{m}$. (J) Sholl analyses for the four groups in $(F-l)$. Note that rapamycin treatment had a milder effect on the dendritic structure in Nedd4-1 KO neurons. $(K)$ Statistical analysis of the total number of crossing neurites obtained in the Sholl analysis shown in $(J)$. Data were analyzed by one-way ANOVA and Tukey's post hoc test ( $N>60$ neurons per group). Data are expressed as mean \pm SEM. $N 1^{\mathrm{f} / \mathrm{f}}$, Nedd4- ${ }^{\mathrm{f} / \mathrm{f}}$. NEX-N1 $1^{\mathrm{f} / \mathrm{f}}, \mathrm{NEX}-\mathrm{Cre} ; \mathrm{Nedd} 4-1^{\mathrm{f} / \mathrm{f}}$.

\subsubsection{Pathways Operating Parallel to the PI3K/PTEN-mTORC1-Nedd4- 1 Signaling Regulates Neurite Growth and Polarity Formation}

To explore the reason as to why there was only a partial rescue of the aberrantly enhanced neurite growth in PTEN KO neurons by additional KO of Nedd4-1 (Figures 3-9 A-E), I first applied rapamycin to control and PTEN KO neurons. Importantly, rapamycin treatment, like additional KO of Nedd4-1, did not completely rescue the neurite hypertrophy phenotype of PTEN KO neurons (Figures 3-10 A-F). This 'rapamycin insensitive fraction' of enhanced neurite growth in PTEN KO neurons may be contributed by pathways that operate in parallel to the PTEN/PI3K-mTORC1 pathway, e.g. the PTEN/PI3K-GSK3 $\beta$ pathway, and are thus not affected by rapamycin treatment or by additional KO of Nedd4-1. Accordingly, it has been reported that GSK3 $\beta$ plays a role in the regulation of dendrite growth (Rui et al., 2013). Secondly, in a subset of experiments shown in Figure 3-10, I performed the rapamycin treatment on PTEN;Nedd4-1 double KO neurons. I found that rapamycin had hardly any effect on neurite growth in PTEN;Nedd4-1 double KO neurons, further supporting the notion that Nedd4-1 operates downstream of mTORC1 (Figures 3-10 G-I, see also Figures 3-9 F-K). Of note, while PTEN KO neurons tend to project more than one axon because of deregulation of another signaling molecule downstream of PTEN, GSK3 $\beta$ (Jiang et al., 2005), this gain-of-function phenotype in axon acquisition was independent of Nedd4-1/Nedd4-2 deletion (Figure 3-11). Taken together, these results of analyzing the neurite complexity and polarity formation of PTEN KO neurons indicate that additional pathways operating parallel to PI3K/PTEN-mTORC1-Nedd4-1 contributes to the neurite growth regulation, 
and these pathways are also responsible for the PTEN-regulated neuronal polarity formation.

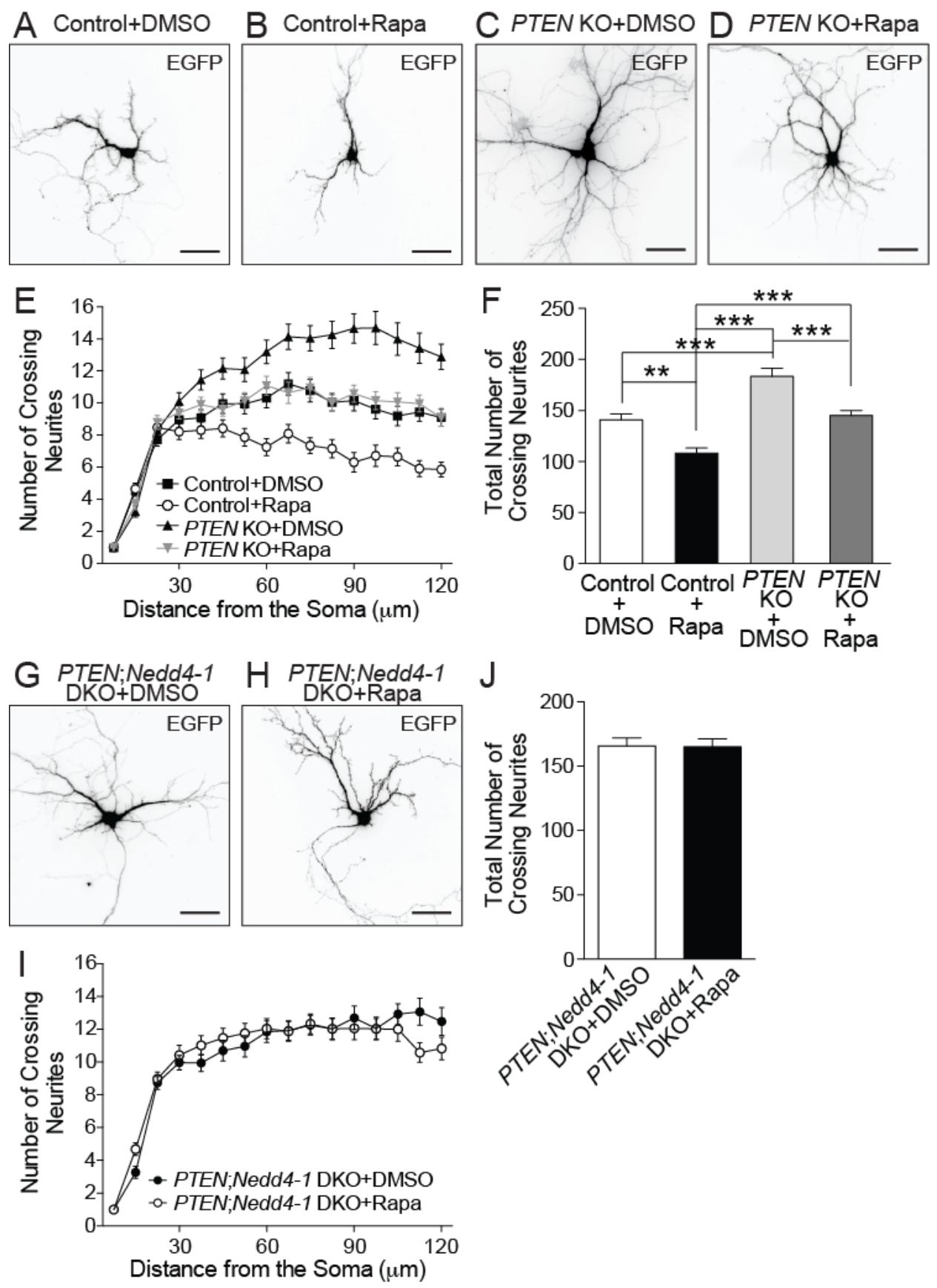

Figure 3-10. Enhanced mTORC1 Activity Partially Accounts for the Hypertrophy of Neurites in PTEN KO Neurons

$(A-D)$ Representative images of a control neuron treated with DMSO $(A)$, a control neuron treated with rapamycin $(B)$, a PTEN KO neuron treated with DMSO $(C)$, and a PTEN KO neuron treated with rapamycin $(D)$. Cultured hippocampal neurons were prepared from $P T E N^{f / f}$ mice and transfected with an EGFP and Cre expressing vector (PTEN KO). Control neurons were 
prepared from littermates and transfected with the EGFP-only expressing vector. Scale bars, $50 \mu \mathrm{m}$. (E) Sholl analyses for the four groups in $(A-D)$. Note the difference between 'PTEN KO + rapa' and 'control + rapa' groups, indicating that pathways independent of mTORC1 activity also contribute to the enhanced neurite growth in PTEN KO neurons. $(F)$ Statistical analysis of the total number of crossing neurites obtained in the Sholl analysis shown in $(E)$. Data were analyzed by one-way ANOVA and Tukey's post hoc test ( $N>49$ neurons per group). ( $G$ and $H$ ) Representative images of a PTEN;Nedd4-1 double KO (DKO) neuron treated with DMSO $(G)$, or with rapamycin $(H)$. Scale bars, $50 \mu \mathrm{m}$. Cultured hippocampal neurons were prepared from littermates with the genotype $P T E N^{\mathrm{fff}} ; N e d d 4-1^{\mathrm{fff}}$, and transfected with an EGFP and Cre expressing vector. (I) Sholl analyses for the two groups in $(G)$ and $(H)$. Note that rapamycin had hardly any effect on dendrite growth in PTEN;Nedd4-1 double KO neurons. (J) Statistical analysis of the total number of crossing neurites obtained in the Sholl analysis shown in $(I)$. Data obtained in $(G-J)$ are from a subset of experiments shown in $(A-F)$. $p=0.9333$ unpaired $t$ test, PTEN;Nedd4-1 DKO+DMSO, N=49; PTEN;Nedd4$1 \mathrm{DKO}+\mathrm{Rapa}, \mathrm{N}=60$. Data are expressed as mean \pm SEM. 
A

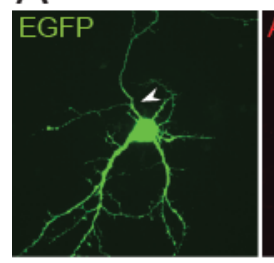

Double KO (Nedd4-1 ${ }^{\mathrm{fff}} ; \mathrm{Nedd} 4-2^{\mathrm{fff}}+\mathrm{Cre}+\mathrm{EGFP}$

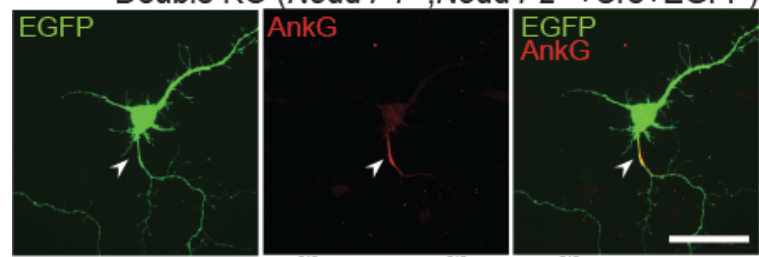

Triple KO (Nedd4-1 ${ }^{\mathrm{fff}} ; \mathrm{Nedd} 4-2^{\mathrm{fff}} ;$ PTEN $\left.\mathrm{N}^{\mathrm{fff}}+\mathrm{Cre}+\mathrm{EGFP}\right)$
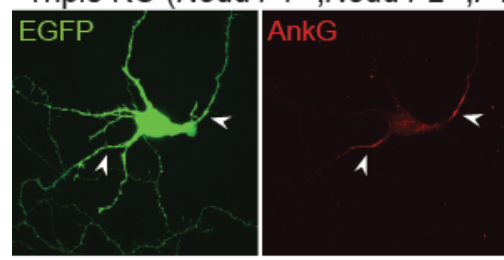

C
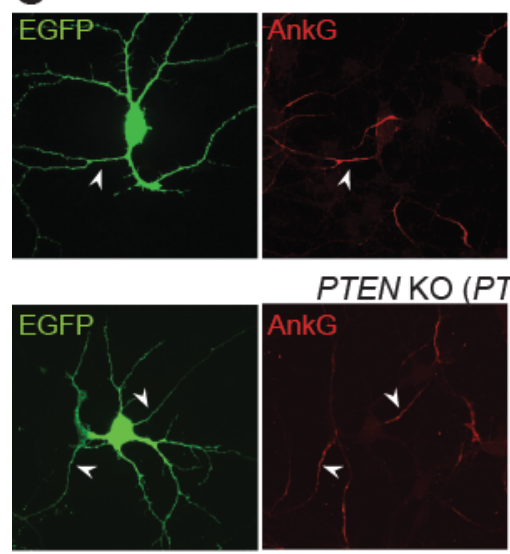

B
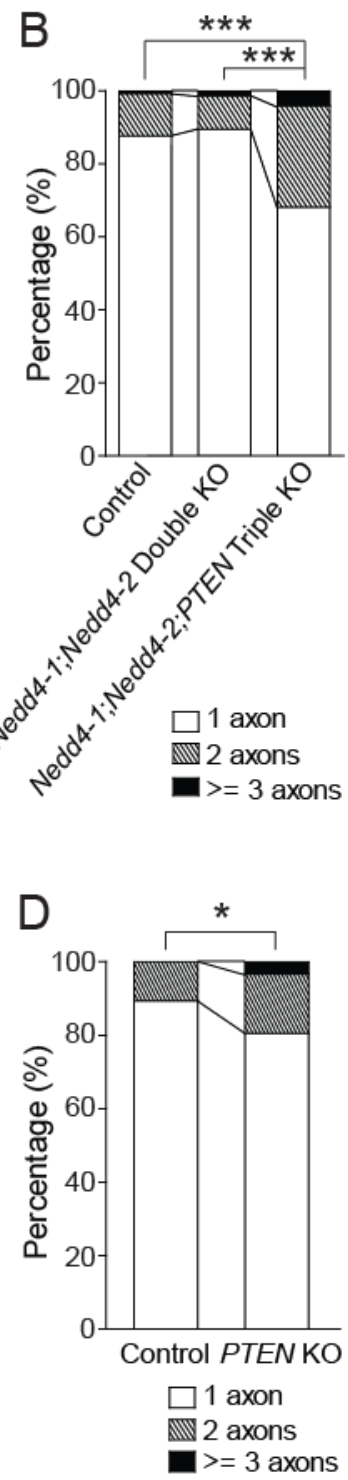

Figure 3-11. Nedd4-1 and Nedd4-2 are Dispensable for PTEN-Mediated Establishment of Neuronal Polarity

(A) Representative images of control (top panels), Nedd4-1;Nedd4-2 double KO (second panels from the top), and Nedd4-1;Nedd4-2;PTEN triple KO neurons (bottom panels). Primary hippocampal neurons prepared from the indicated genotypes were transfected with an EGFP expressing vector or an EGFP and Cre co-expressing vector at DIV1 and fixed at DIV7. Staining for AnkyrinG (AnkG) was used to label axon initial segments. Scale bars, $50 \mu \mathrm{m}$. $(B)$ Statistical analysis of the number of axons for the three groups in $(A)$. The percentage of neurons projecting multiple axons was increased in Nedd4-1;Nedd4-2;PTEN triple KO neurons as compared to Nedd4-1;Nedd42 double KO neurons $\left(^{* * *} p<0.0001\right.$, chi-square test) and control neurons $\left({ }^{* * *} p=0.0005\right.$, chi-square test). Nedd4-1;Nedd4-2 double KO neurons, on the other hand, showed no significant difference as compared to control neurons. $N>120$ neurons per group. (C) Representative images of control (top panels), and PTEN KO neurons (bottom panels). Scale bars, $50 \mu \mathrm{m}$. (D) Statistical 
analysis of the number of axons for the two groups in $(C)$. A significantly increased percentage of PTEN KO neurons projected multiple axons as compared to control neurons. ${ }^{*} p=0.0394$, chi-square test. Control, $\mathrm{N}=123$; PTEN KO, $\mathrm{N}=115$. Data are expressed as mean \pm SEM.

\subsubsection{Working Model}

In the present study, I demonstrate that the Nedd4 family E3 ubiquitin ligases, Nedd4-1 and Nedd4-2, promote axon growth in mammalian central nervous system (CNS) neurons independently of PTEN acting as a downstream substrate. Instead, PTEN acts upstream of Nedd4-1 to negatively regulate Nedd4-1 expression at the translational level via mTORC1 to control neurite growth (Figure 3-12)

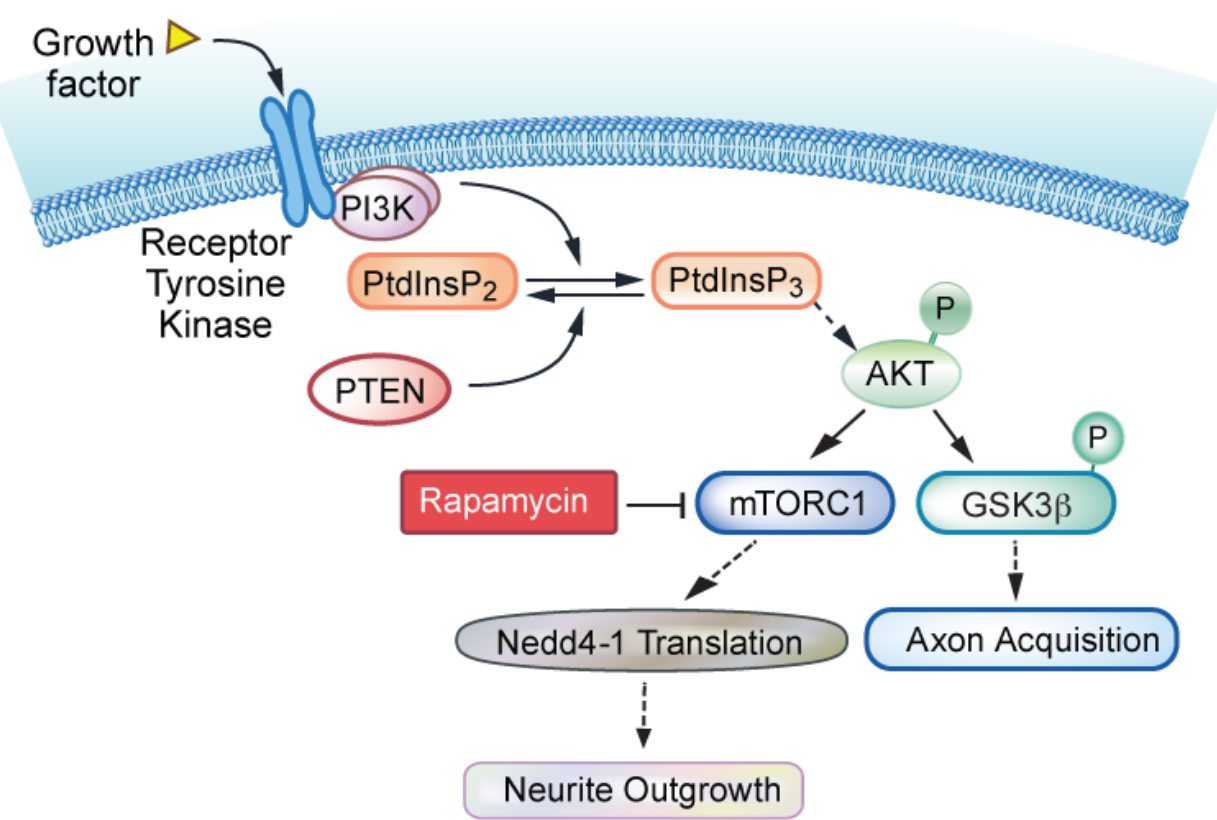

Figure 3-12. Model of the Regulation of Nedd4-1 by the PI3K/PTENmTORC1 Signaling Pathway during Neurite Growth

$\mathrm{PI} 3 \mathrm{~K}$ catalyzes the phosphorylation of $\mathrm{Ptdlns}_{2}$ to generate $\mathrm{Ptdlns}_{3}$ (e.g. in response to growth factor or hormone stimulation). PTEN converts Ptdlns $\mathrm{P}_{3}$ back to PtdlnP 2 and thus antagonizes the effect of PI3K. Elevated Ptdlns $\mathrm{P}_{3}$ levels lead to the phosphorylation of AKT, which further phosphorylates GSK3 $\beta$ and mTORC1. The phosphorylation of GSK3 $\beta$ promotes axon acquisition in neurons (Jiang et al., 2005). On the other hand, AKT dependent activation of mTORC1 stimulates downstream pathways that include the protein translation machinery. Nedd4-1 is important for neurite outgrowth but dispensable for axon acquisition, and its mRNA is a prominent 
target of regulation by $\mathrm{mTORC} 1$ at the translational level. Nedd4-1 is not responsible for PTEN ubiquitination or regulation in developing mammalian neurons.

\subsection{Roles of the E3 Ligases WWP1 and WWP2 during Mammalian Brain Development}

\subsubsection{Identification of Binding Partners of WWP1}

To study the roles of WWP1 in brain development, I first affinity-purified WWP1 binding proteins from P7 mouse brains. Recombinant GST-tagged full-length WWP1 WT (GST-WWP1 WT) was purified from E. coli and immobilized on glutathione sepharose beads, followed by incubation with an extract of brain synaptosomes prepared from P7 WT mice. Proteins bound to the beads were subsequently eluted with buffer containing $1 \mathrm{M} \mathrm{NaCl}$, followed by $40 \mathrm{mM}$ glutathione (Figure 3-13). Using mass spectrometric methods in collaboration with Dr. Olaf Jahn (Proteomics Group, MPI-EM, Göttingen), we identified several proteins that were specifically enriched in the eluate from a GST-WWP1 column loaded with brain lysates (Table 3-1). Of note, one of the candidates was shown on Coomassie-stained gels as a clearly distinct band and was resistant to $1 \mathrm{M} \mathrm{NaCl}$ elution (Figure 3-13, number 1), indicating a strong affinity between this candidate protein and WWP1. The subsequent mass spectrometric analyses identified this protein with high confidence as 'probable ubiquitin carboxyl-terminal hydrolase (Usp9x)'. The interaction between Usp9x and WWP1/WWP2 was further studied by immobilizing recombinant GST-WWP1 WT and GST-WWP2 WT on columns, followed by loading the columns with lysates prepared from HEK293FT cells overexpressing V5-Usp9x. The interaction between V5Usp9x and WWP1/WWP2 were confirmed by Western blotting using an antibody to V5, which detected specific signals of V5-Usp9x for the GSTWWP1 and GST-WWP2 column eluates but not for the negative control (Figure 3-14, top blots). I therefore focused on studying a possible functional interaction between Usp9x and WWP1/WWP2. 


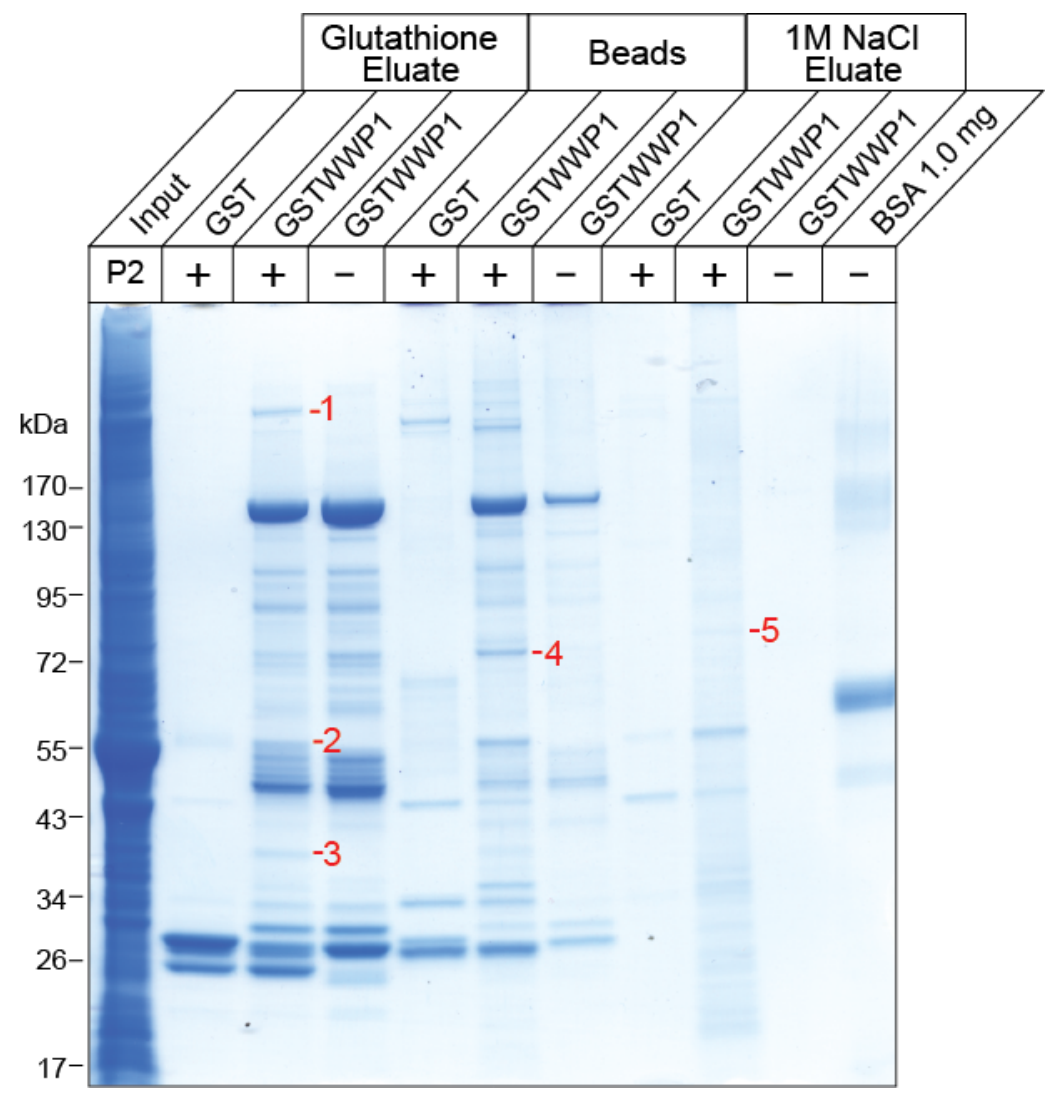

Figure 3-13. Affinity Purification of WWP1 Binding Proteins from P7 Mouse Brains

GST-WWP1 binding proteins were affinity-purified from an extract of brain synaptosomes (P2) prepared from P7 WT mice. A GST column loaded with extract of brain synaptosomes and a GST-WWP1 column only loaded with buffer were used as negative controls. Proteins were separated on a pre-cast NuPAGE 4\%-12\% Bis-Tris gradient gel (Invitrogen) using the MOPS-based buffer system, and visualized by Coomassie staining. Several individual bands specifically enriched by the GST-WWP1 column loaded with extract of brain synaptosomes (indicated by numbers) were excised for further analysis using mass spectrometry. Note that the band number 1 was shown as a clearly distinct band that was absent in negative controls and resistant to $1 \mathrm{M}$ $\mathrm{NaCl}$ elution. 
Table 3-1. List of Brain Specific WWP1 Binding Proteins Identified by Mass Spectrometry

\begin{tabular}{ccc}
\hline Number\# & Protein Name & Accession Number \\
\hline 1 & $\begin{array}{c}\text { Probable ubiquitin carboxyl-terminal hydrolase } \\
\text { FAF-X (Usp9x) }\end{array}$ & gil115511018 \\
\hline 2 & Tubulin beta-2C chain & gi|5174735 \\
\hline 3 & WW domain-binding protein 2 & gi|1110559 \\
\hline 4 & Cleavage and polyadenylation specificity factor & gi|26338454 \\
\hline 5 & Heat shock protein 70 cognate & gi|309319 \\
\hline
\end{tabular}

\#Numbers correspond to bands indicated in Figure 3-13

Usp9x was reported as the deubiquitinase that removes polyubiquitin chains from the AMPK-related kinase Nuak1 and thereby activates the kinase activity of Nuak1 (Al-Hakim et al., 2008). During mammalian axon morphogenesis, Nuak1 is phosphorylated and activated by LKB1 to promote axonal branching of cortical neurons (Courchet et al., 2013). Thus, I investigated the possibility that WWP1 also targets Nuak1 and antagonizes the function of Usp9x by acting upon the same effector protein. To test this, I studied if Nuak1 and its closest homologue Nuak2 interact with WWP1 or WWP2. Lysates prepared from HEK293FT cells overexpressing Flag-Nuak1 or HA-Nuak2 were loaded onto a GST-WWP1 or a GST-WWP2 column and the bound fractions were analyzed by Western blotting using antibodies to Flag and HA, respectively. I found specific interactions between both Nuak1 and Nuak2 with WWP1/WWP2 (Figure 3-14, middle and bottom blots), indicating that similar to Usp9x, WWP1 and WWP2 may also play roles in regulating the functions of Nuak1 or Nuak2. 


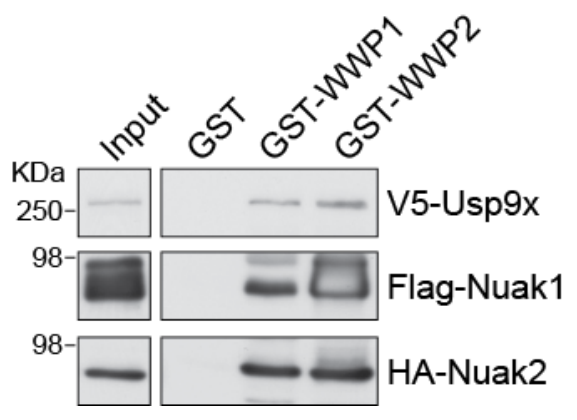

Figure 3-14. Interactions of Usp9x, Nuak1, and Nuak2 with WWP1 and WWP2

Lysates prepared from HEK293FT cells overexpressing V5-Usp9x, FlagNuak1, or HA-Nuak2 were loaded on GST, GST-WWP1 or GST-WWP2 columns. Bound fractions were subjected to Western blotting using antibodies for V5, Flag, or HA, respectively. Note that there is specific binding of Usp9x, Nuak1, and Nuak2 to both WWP1 and WWP2.

\subsubsection{Proteomic Based Screening of Ubiquitination Substrates of WWP1 and WWP2 in Mouse Brains}

In addition to the identification of WWP1 and WWP2 binding proteins, which may potentially be ubiquitination substrates of WWP1 or WWP2, I also used a proteomics based screening service provided by Cell Signaling Technology (PTMscan) to directly screen for ubiquitination substrates in an unbiased manner. Briefly, brain lysates were prepared from control and EmxCre;WWP $1^{\mathrm{f} / f} ; W W P 2^{\mathrm{f} / \mathrm{f}}$ mice and proteins were trypsinized. The resulting peptides with di-glycine remnants from ubiquitin left on the lysine residues

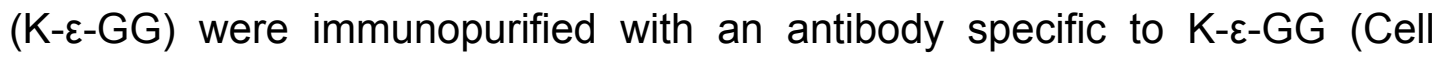

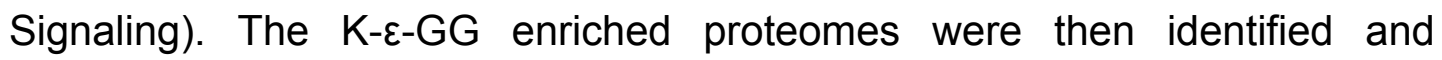
quantified using mass spectrometry (see also Figure 1-3). Several proteins showing a robust decreased ubiquitination level in Emx-Cre;WWP ${ }^{\mathrm{f} / \mathrm{f}} ; W W P 2^{\mathrm{f} / \mathrm{f}}$ brains as compared to control brains appear to be potential substrates of WWP1 or WWP2 (Table 3-2). I further validated the proteomics results with an in vitro ubiquitination assay using recombinant E1, E2s (both from Boston Biochem), E3s (purified from E. coli) and the candidate proteins as substrates. Candidate proteins were either overexpressed with an $\mathrm{N}$-terminal Myc-tag in HEK293FT cells and immunopurified using anti-Myc agarose beads, or overexpressed with an N-terminal GST-tag in E. coli and purified 
with glutathione sepharose beads, followed by removing the GST-tag with thrombin or prescission protease. Of the candidate proteins I tested, Tuba4A, UBE1, Pkar1b, and Cdk5 were ubiquitinated by the WWP ligases in vitro, but MEKK6 was not (Figure 3-15). These proteomics and in vitro ubiquitination results indicate that Tuba4A, UBE1, Pkar1b, and Cdk5 are prominent substrates of WWP1/WWP2 in the developing mammalian brain.

Table 3-2. List of Potential Substrate Proteins of WWP1 and WWP2 Identified in the Proteomic Screening

\begin{tabular}{ccccc}
\hline $\begin{array}{c}\text { Normalized } \\
\text { Fold Change } \\
\text { (KO/Control) }\end{array}$ & $\begin{array}{c}\text { Intensity } \\
\text { (Control) }\end{array}$ & $\begin{array}{c}\text { Gene } \\
\text { Name }\end{array}$ & Protein Name & $\begin{array}{c}\text { Uniprot } \\
\text { Accession } \\
\text { Number }\end{array}$ \\
\hline-4.0 & 466.759 & $\begin{array}{c}\text { Cdk5; } \\
\text { Map3k6 }\end{array}$ & Cdk5; MEKK6 & $\begin{array}{c}\text { P49615; } \\
\text { Q9WTR2 }\end{array}$ \\
\hline-3.1 & 1215.700 & $\begin{array}{c}\text { Slbp; } \\
\text { Tuba1b; } \\
\text { Tuba4a }\end{array}$ & $\begin{array}{c}\text { Tuba3C; } \\
\text { Tuba1B; }\end{array}$ & $\begin{array}{c}\text { XP_486246; } \\
\text { P05213; }\end{array}$ \\
\hline-2.5 & 6714.891 & Uba1 & UBE1 & Q68368 \\
\hline-2.2 & 893.872 & Prkar1b & Pkar1b & P12849 \\
\hline
\end{tabular}

Cdk5, cyclin-dependent kinase 5; MEKK6, mitogen-activated protein kinase kinase kinase 6; Tuba3C, tubulin alpha-3C; Tuba1B, tubulin alpha-1B; Tuba4A, tubulin alpha-4A; UBE1, ubiquitin-activating enzyme 1; Pkar1b, cAMP-dependent protein kinase type I-beta regulatory subunit. 
Western blotting: Myc

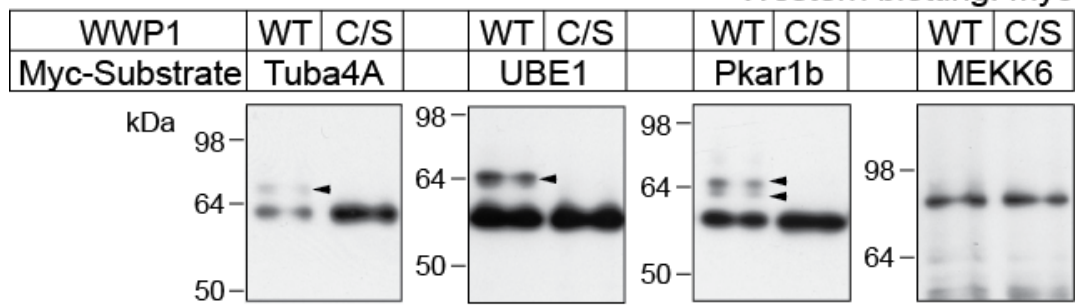

Western blotting: Cdk5

\begin{tabular}{|c|c|c|c|c|c|}
\hline E2 & + & + & - & + & - \\
\hline WWP1 & WT & C/S & WT & - & - \\
\hline WWP2 & - & - & - & WT & - \\
\hline
\end{tabular}

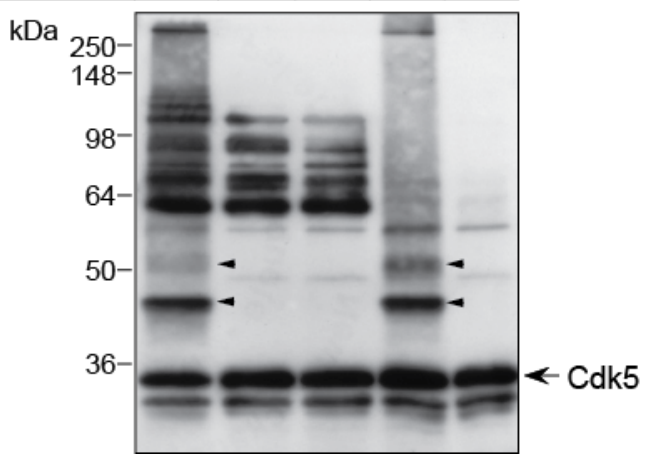

Figure 3-15. Validation of the Proteomic Screening Results

Potential substrate proteins of WWP1/WWP2 identified in the proteomic screening (Table 3-2) were validated using an in vitro ubiquitination assay. The recombinant candidate proteins were either purified from HEK293FT cells (Tuba4A, UBE1, Pkar1b, and MEKK6) or E. coli (Cdk5). The results were analyzed by Western blotting using the indicated antibodies. Note that Tuba4A, UBE1, and Pkar1b were mono- and di-ubiquitinated by WWP1, and Cdk5 was poly-ubiquitinated by WWP1 and WWP2 in vitro. MEKK6 was not a substrate of WWP1 in vitro. The mono- and di-ubiquitinated forms of candidate proteins are indicated by arrowheads.

\subsubsection{Cdk5 is a Physiological Substrate of WWP1 and WWP2}

Numerous reports indicated that Cdk5 plays important roles in mammalian neural development (reviewed by Dhavan and Tsai, 2001; Su and Tsai, 2011). I therefore focused on studying if Cdk5 is a physiological substrate of WWP1 and WWP2. To test if Cdk5 is ubiquitinated by WWP1 and WWP2 in vivo, I overexpressed Myc-Cdk5 together with WWP1 or WWP2 in HEK293FT cells. Myc-Cdk5 was then immunoprecipitated from the cell lysates under denaturing conditions using anti-Myc agarose beads (Sigma), and its ubiquitination level was studied by Western blotting to 
ubiquitin. Of note, Cdk5 was ubiquitinated by WWP1 and WWP2 in vivo, since the higher molecular weight smear pattern detected in the anti-ubiquitin blot was increased in samples with co-expressed WWP1 or WWP2, as compared to the ligases only or the Myc-Cdk5 only negative controls (Figure $3-16 A)$. This increase in the higher molecular weight smear pattern was also apparent in the anti-Myc blot (Figure 3-16A). In addition, I investigated if the ubiquitination level of Cdk5 is reduced in WWP1;WWP2 KO neurons as compared to control neurons. Myc-Cdk5 was overexpressed in cultured control and WWP1;WWP2 KO hippocampal neurons using the lentivirus system, followed by an anti-Myc immunoprecipitation under denaturing conditions and Western blotting to ubiquitin. Strikingly, deletion of WWP1/WWP2 in neurons reduced the ubiquitination level of Cdk5 (Figure 316B). Together, the data obtained in HEK293FT cells and neurons indicate that Cdk5 is a physiological ubiquitination substrate of WWP1/WWP2.

Given that Cdk5 has a PY motif that was reported to be important for binding to the WW-domains of Nedd4 family ligases (reviewed by Rotin and Kumar, 2009), I further studied if Cdk5 directly interacts with WWP1 and WWP2 and if these interactions are dependent on the PY motif of Cdk5. For this purpose, I immobilized recombinant GST-Cdk5 WT and GST-Cdk5 PY motif mutant (Cdk5 P234A, Y236F) on columns and loaded the columns with recombinant full-length WWP1 WT or WWP2 WT purified form E. coli. The bound fractions were collected and analyzed by Western blotting using antiWWP1 or anti-WWP2 antibodies. Interestingly, both WWP1 and WWP2 bound to Cdk5 WT but the affinities to the Cdk5 PY motif mutant were weaker, indicating that WWP1/WWP2 interact directly with Cdk5 and that such interactions are mediated via the PY motif of Cdk5 (Figure 3-16C). 
A

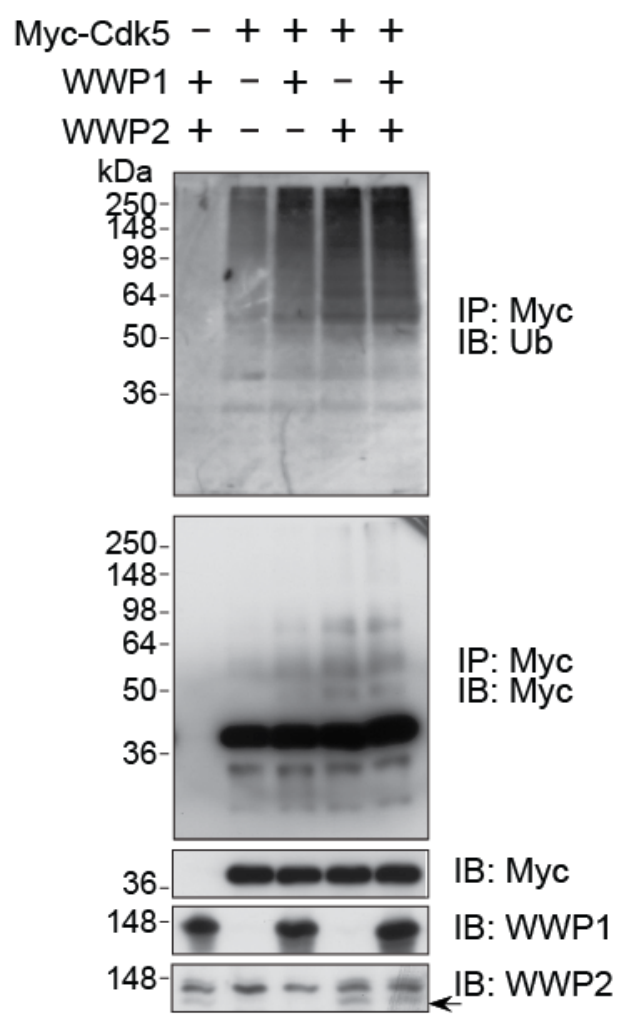

B

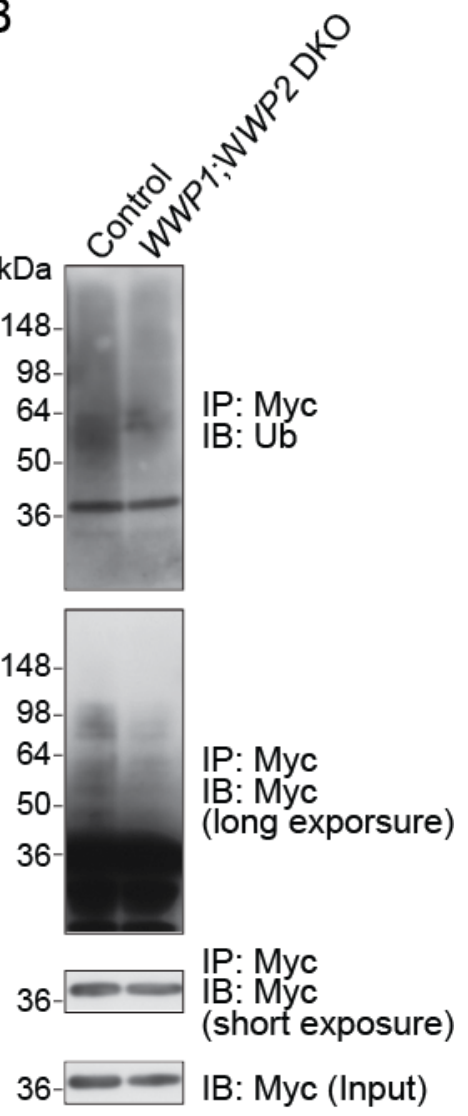

C
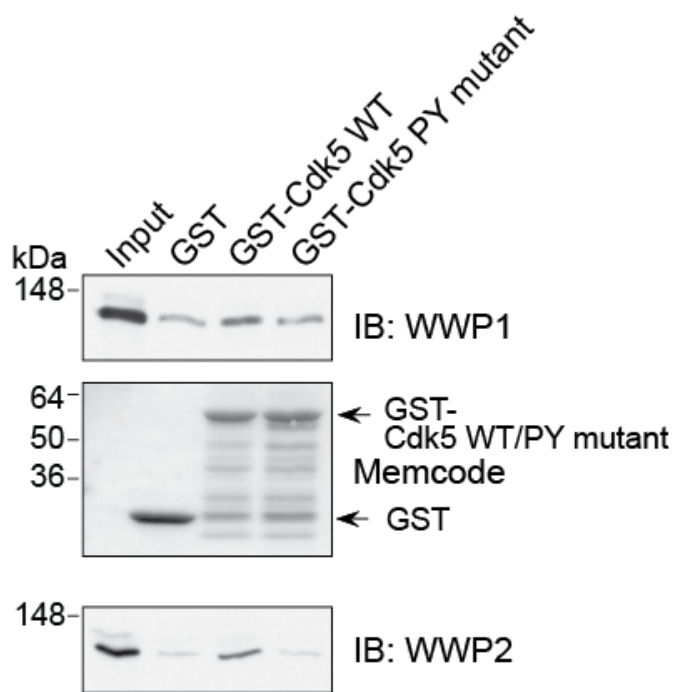

IB: WWP2

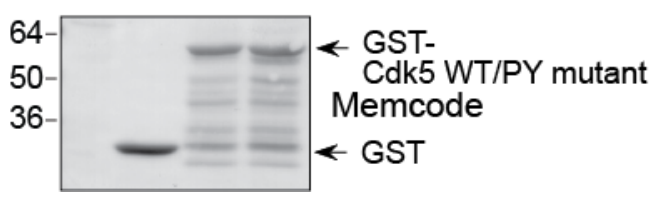

Figure 3-16. Cdk5 Interacts Directly with WWP1/WWP2 and is a Physiological Substrate of WWP1/WWP2

(A) Cdk5 is ubiquitinated by WEMBO Molecular MedicineWP1/WWP2 in vivo. Myc-Cdk5 was overexpressed in HEK293FT cells together with WWP1 or WWP2, immunoprecipitated with anti-Myc agarose beads, and its 
ubiquitination level was studied by Western blotting to ubiquitin. Note that the smear patterns shown in the anti-ubiquitin blot and the anti-Myc blot are increased when Myc-cdk5 was co-expressed with WWP1 or WWP2, as compared to the negative controls (first two lanes). Specific bands for overexpressed WWP2 are indicated by the arrow. (B) Reduced ubiquitination level of Cdk5 in WWP1;WWP2 DKO neurons. Cultured hippocampal neurons were prepared from $W W P 1^{\mathrm{f} / f} ; W W P 2^{\mathrm{f} / \mathrm{f}}$ mice, and vectors expressing EGFP (Control) or EGFP and Cre (DKO) were expressed using lentiviral infection at DIV0. Myc-Cdk5 was overexpressed in control and WWP1;WWP2 KO neurons using lentiviral infection at DIV1. Neurons were harvested at DIV14. Myc-Cdk5 was immunoprecipitated with anti-Myc agarose beads and its ubiquitination level was studied by Western blotting to ubiquitin. (C) Cdk5 directly binds to WWP1/WWP2 via the PY motif of Cdk5. $45 \mu \mathrm{g}$ of recombinant GST-Cdk5 WT and GST-Cdk5 PY mutant (P234A, Y236F) was immobilized on glutathione sepharose beads, and incubated with $10 \mu \mathrm{g}$ of recombinant full-length WWP1 or WWP2. The bound fractions were analyzed by Western blotting using anti-WWP1 and anti-WWP2 antibodies (first and third panels). Note that both WWP1 and WWP2 specifically bind to Cdk5 WT, but the affinities are weaker to the Cdk5 PY motif mutant. Similar amounts of immobilized GST and GST-fusion proteins were confirmed by the Memcode (Pierce) staining (second and fourth panels). IB, immunoblotting. IP, immunoprecipitation. Ub, ubiquitin.

\subsubsection{Regulation of Cdk5 by WWP1/WWP2 Mediated Ubiquitination}

Next, I investigated the possible roles of Cdk5 ubiquitination by WWP1 and WWP2. I first studied whether the polyubiquitin chains conjugated on Cdk5 by the WWP ligases are K48-linked or K63-linked, given that K48linked polyubiquitination targets the substrate protein for proteosomal degradation, whereas K63-linked polyubiquitination plays roles in other regulatory processes (reviewed by Ikeda and Dikic, 2008). For this purpose, Myc-Cdk5 was overexpressed in HEK293FT cells together with WWP1 or WWP2, and either HA-ubiquitin WT, HA-ubiquitin K48R, or HA-ubiquitin K63R. Myc-Cdk5 was then immunoprecipitated under denaturing conditions with anti-Myc agarose beads, and its ubiquitination level was studied by Western blotting using an antibody to HA. Interestingly, both WWP1 and WWP2 mainly conjugated K63-linked polyubiquitin chains but not K48-linked polyubiquitin chains to Cdk5, as indicated by the fact that co-expression of the HA-ubiquitin K63R mutant reduced the polyubiquitination level of Cdk5, whereas co-expression of the HA-ubiquitin K48R had no effect on the Cdk5 
ubiquitination level as compared to co-expression of the HA-ubiquitin WT (Figure 3-17 $A$ and $B$ ). These results indicate that WWP1/WWP2 do not regulate the proteasomal degradation of Cdk5 by conjugating K48-linked polyubiquitin chains. Rather, WWP1/WWP2 may regulate the function or subcellular localization of Cdk5 by conjugating K63-linked polyubiquitin chains on Cdk5.

Activation of the kinase activity of Cdk5 requires binding of Cdk5 with its activators, p35 or p39 (Ko et al., 2001; Tsai et al., 1994). To study if the binding of Cdk5 with p25, an N-terminally truncated form of p35, interferes with the ubiquitination of Cdk5 by WWP1/WWP2, I overexpressed Myc-Cdk5 together with or without HA-p25 in HEK293FT cells. Myc-Cdk5 was then immunopurified using anti-Myc agarose beads and subjected to an in vitro ubiquitination assay. Strikingly, co-expression of HA-p25 reduced the ubiquitination efficiency of Myc-Cdk5 by WWP1 in vitro, indicating that binding with p25 hinders ubiquitination of Cdk5 by WWP1 (Figure 3-17C). This result is also compatible with the notion that Cdk5 ubiquitination by WWP ligases may hinder the binding of Cdk5 with its activators, and thereby interfere with the activation of Cdk5. Taken together, my current results indicate that ubiquitination of Cdk5 by WWP ligases may regulate the kinase activity of Cdk5. 
A

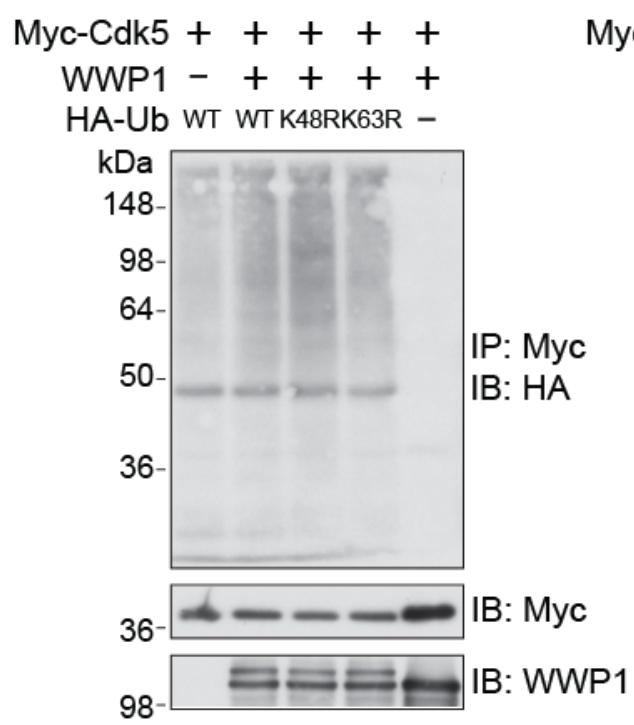

B

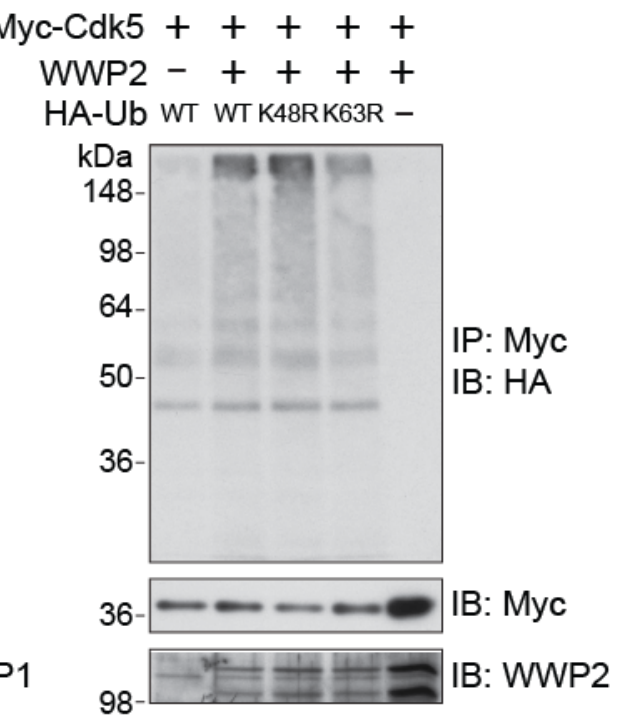

C

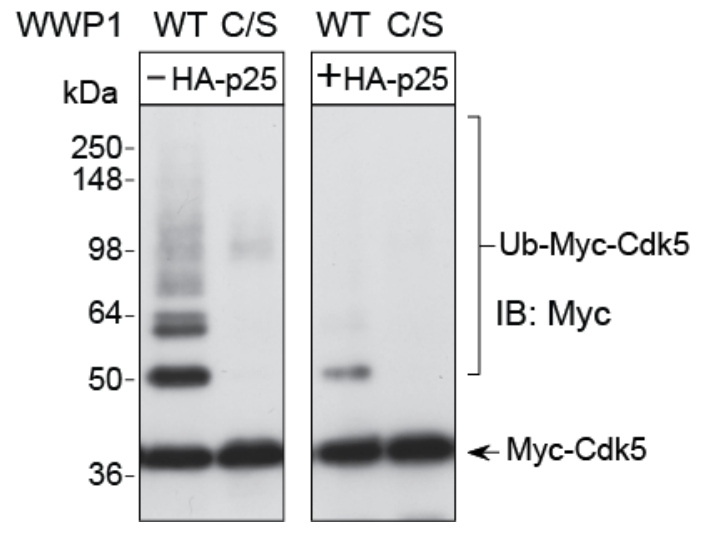

Figure 3-17 Regulation of Cdk5 by WWP Ligase-Mediated Ubiquitination $(A$ and $B$ ) WWP1 and WWP2 mainly conjugate K63-linked polyubiquitin chains rather than K48-linked polyubiquitin chains to Cdk5. Myc-Cdk5 was overexpressed in HEK293FT cells together with WWP1 $(A)$ or WWP2 $(B)$, and either HA-ubiquitin WT, HA-ubiquitin K48R, or HA-ubiquitin K63R. MycCdk5 was then immunoprecipitated and its ubiquitination level was studied by Western blotting to ubiquitin. Note that for both WWP1 and WWP2, the smear patterns of polyubiquitination of Myc-Cdk5 were reduced if coexpressed with HA-ubiquitin K63R. (C) Binding of Cdk5 with its activator p25 reduces ubiquitination of Cdk5 by WWP1. Myc-Cdk5 was overexpressed in HEK293FT cells together with (left blot) or without (right blot) HA-p25, immunoprecipitated with anti-Myc agarose beads, and subjected to an in vitro ubiquitination assay using WWP1 as the E3 ligase. Note that the ubiquitination efficiency of Cdk5 by WWP1 is reduced when HA-p25 was coexpressed. Shown results were obtained from the same set of experiments on the same blot. IB, immunoblotting. IP, immunoprecipitation. Ub, ubiquitin. 


\section{Discussion}

\subsection{PTEN is Not a Relevant Substrate of Mammalian Nedd4 Family E3 Ligases in the Regulation of Neurite Development}

Nedd4-1 is the first ubiquitin E3 ligase to be implicated in the mono- and polyubiquitination of PTEN (Wang et al., 2007). Based on this initial study, it was proposed that Nedd4-1 regulates the expression level, phosphatase activity, and nuclear import of PTEN in various cell types (Ahn et al., 2008; Christie et al., 2012; Chung et al., 2011; Guo et al., 2012; Kwak et al., 2010; Trotman et al., 2007; Yim et al., 2009). However, the issue of whether PTEN is ubiquitinated and regulated by Nedd4-1 is highly controversial, given that other groups reported that PTEN protein expression and subcellular distribution are not altered in Nedd4-1 KO or Nedd4-1 KD cells (Fouladkou et al., 2008; Cao et al., 2008; Yang et al., 2008a). Several other groups also identified alternative E3 ligases targeting PTEN, including XIAP, WWP2, and CHIP (Ahmed et al., 2012; Maddika et al., 2011; Van Themsche et al., 2009), suggesting the possibility that the regulation of PTEN by ubiquitination may be cell type specific or context dependent.

In the postmitotic developing neurons, mouse Nedd4-1 promotes dendritic growth in hippocampal and cortical neurons by monoubiquitinating the small GTPase Rap2A (Kawabe et al., 2010), whereas xNedd4, which is more homologous to mammalian Nedd4-2, promotes axonal branching in Xenopus laevis retinal ganglion neurons by polyubiquitinating PTEN and target it for proteosomal degradation (Drinjakovic et al., 2010). The conclusions regarding the role of $\mathrm{xNedd} 4$ in Xenopus laevis retinal ganglion cells were mainly based on results showing that (i) axonal branching of retinal ganglion cells is inhibited upon overexpression of dominant negative xNedd4 or KD of xNedd4; (ii) PTEN levels are increased in axonal growth cones of dominant negative xNedd4 overexpressing or xNedd4 KD neurons; (iii) the impairment in axonal branching upon $x$ Nedd4 $\mathrm{KD}$ is rescued by 
simultaneous downregulation of endogenous PTEN; (iv) overexpression of a ubiquitin K48R mutant also impairs the axonal branching in retinal ganglion cells; and (v) application of a proteasome inhibitor blocks the Netrin-1induced PTEN degradation and growth cone collapse (Drinjakovic et al., 2010). This study on the roles of $x$ Nedd4 in PTEN regulation has challenged the finding that PTEN expression levels were unaltered in Nedd4-1 KO mouse embryonic fibroblasts (Fouladkou et al., 2008). The latter paper, nevertheless, does not exclude the possibility that loss of Nedd4-1 is compensated by its closest mammalian homologue Nedd4-2.

In the present study, I tackle this highly contentious issue of whether PTEN is ubiquitinated by Nedd4-1 or Nedd4-2 in mammalian CNS neurons by using the brain specific Nedd4-1 single, Nedd4-2 single, and Nedd41;Nedd4-2 double conditional KO mouse lines, which are the cleanest tools available for this purpose. I found that Nedd4-2 has a similar function as Nedd4-1 in the regulation of neurite branching (Kawabe et al., 2010 and Figure 3-1), and that Nedd4-1/Nedd4-2 deletion causes defects in axonal morphogenesis of mouse hippocampal and cortical neurons in a manner similar to what has been observed in Xenopus laevis retinal ganglion cells (Figures 3-3 and 3-4). However, I found no concomitant misregulation of PTEN in Nedd4-1;Nedd4-2 double KO neurons. My data clearly show that alterations in PTEN levels, localization, or function cannot be involved in the regulation of axon growth by Nedd4 family E3 ligases (Figures 3-5, 3-6, and 3-7). These data indicate that the results observed upon perturbation of endogenous xNedd4 functions by overexpressing the dominant negative xNedd4 or using a single sequence morpholino-based xNedd4 KD may not be sufficiently conclusive, as some off-target effects might occur. Particularly, the result of simultaneous KD of PTEN and $x$ Nedd4 rescue the impaired axonal branching phenotype upon xNedd4 KD does not offer a causal relationship between PTEN and xNedd4 (Drinjakovic et al., 2010). Correspondingly, I found that PTEN operates upstream to negatively regulate Nedd4-1 expression, which can also account for their observed results (Figure 3-8). In addition, the finding that PTEN is conjugated with K48-linked 
polyubiquitin chains by $\mathrm{xNedd} 4$ is questionable, as multiple publications have indicated that Nedd4 superfamily E3 ligases mainly conjugate K63-linked polyubiquitin chains for non-proteolytic purposes (Kamadurai et al., 2013; Kim and Huibregtse, 2009).

Although I found no evidence that PTEN is regulated by Nedd41/Nedd4-2 during the development of mammalian CNS neurons, based on the current dataset I cannot exclude the possibilities that PTEN ubiquitination by Nedd4 family E3 ligases is only functionally relevant in specific neuron types such as Xenopus laevis retinal ganglion cells and mammalian peripheral neurons (Drinjakovic et al., 2010; Christie et al., 2012), or that ubiquitination of PTEN only occurs in specific contexts such as neuronal protection upon ischemic stress or zinc insult (Howitt et al., 2012; Kwak et al., 2010). However, in the report showing that Nedd4-1/Nedd4-2 may be relevant for PTEN ubiquitination and nuclear import for neuronal protection upon ischemic stress, the conclusion was mainly based on the observation that PTEN localization is altered in Nedd4 family-interacting protein 1 (Ndfip1) KO mouse brains (Howitt et al., 2012). Ndfip1 is an adaptor protein for the Nedd4 superfamily E3 ligases, and may act as an activator for the Nedd4 ligases by releasing them from the autoinhibitory conformation (Mund and Pelham, 2009). Nevertheless, no direct evidence was obtained that the activity of Nedd4-1 or Nedd4-2 is affected in Ndfip1 KO mouse brains (Howitt et al., 2012). Furthermore, subsequent studies on Ndfip1 KO mice by the same group also indicated that Ndfip1 is important for the export of PTEN to exosomes independently of Nedd4-1 or Nedd4-2 (Putz et al., 2012), and that $\mathrm{KO}$ of Ndfip1 in embryonic cultured neurons and developing brains results in increased complexity of neurites and elevated p-AKT (S473) levels (Hammond et al., 2013), which are opposite to the phenotypes seen in Nedd4-1;Nedd4-2 KO mice (Kawabe et al., 2010 and Figures 3-1, 3-3, 3-4, and 3-5 $E$ and $F$ ). These results indicate that Ndfip1 may have a role in regulating PTEN independent of the Nedd4-1 or Nedd4-2 mediated PTEN ubiquitination. 


\subsection{PTEN Negatively Regulates Nedd4-1 Expression at the Translational Level}

\subsubsection{Nedd4-1 is a Major Target of the PI3K/PTEN-mTORC1 Signaling in Neurite Development}

Beyond merely excluding a role of Nedd4 family E3 ligases in the regulation of PTEN levels, localization, or function in mammalian CNS neurons, my present work also provides evidence for a strikingly inverse scenario, according to which PTEN antagonizes the PI3K-mTORC1 pathway to limit Nedd4-1 mRNA translation and neurite growth. Importantly, my working model (Figure 3-12), in which PTEN acts as an upstream negative regulator of Nedd4-1, can also account for the inverse correlation between expression levels of PTEN and Nedd4-1 found in previous studies (Ahn et al., 2008; Amodio et al., 2010; Kwak et al., 2010; Wang et al., 2007). However, these findings also raise the question as to why Nedd4-1 mRNA is preferentially targeted by the mTORC1 signaling.

Activation of the PI3K-mTORC1 signaling promotes protein synthesis mainly by mediating the phosphorylation of eukaryotic initiation factor $4 \mathrm{E}$ binding protein 1 and 2 (4E-BP1 and 4E-BP2). Under basal conditions, 4E$\mathrm{BPs}$ bind to eukaryotic initiation factor 4E (elF4E) to prevent the formation of protein initiation complex and protein translation. Phosphorylation of $4 \mathrm{E}-\mathrm{BPs}$ leads to decreased affinity of $4 \mathrm{E}-\mathrm{BPs}$ to elF4E, enabling elF4E to be anchored to the 5 '-cap structure of mRNAs and the formation of protein initiation complex to start protein synthesis (reviewed by Gingras et al., 1999). PTEN suppresses this process by counteracting PI3K-mTORC1 activation, and thereby negatively regulates the translation of Nedd4-1 mRNA (Figure 3-7). Further data supporting this regulation include a published study employing ribosome profiling to examine the translational efficiency of specific mRNAs in the presence of an mTORC1 inhibitor, and showing that the translational efficiency of Nedd4-1 mRNA is reduced upon mTORC1 inhibition (Thoreen et al., 2012). Of note, the 5 ' untranslated region 
(5'UTR) of Nedd4-1 mRNA (GenBank accession number: NM_010890) contains a pyrimidine-rich sequence stretch that is related to the $5^{\prime}$ terminal oligopyrimidine (5'TOP) motif. This motif is important for anchoring elF4E to the 5'-cap structure of the mTORC1 target mRNAs to initiate protein synthesis upon phosphorylation of $4 \mathrm{E}-\mathrm{BPs}$. The pyrimidine-rich sequence in the 5'UTR of Nedd4-1 mRNA may therefore play a similar role as the 5'TOP motif in starting translation of Nedd4-1 mRNA in a mTORC1 activitydependent manner.

In addition to the biochemical results showing that PTEN acts as an upstream regulator to negatively regulate Nedd4-1 translation (Figure 3-7), I also provide evidences indicating that Nedd4-1 is a major target of the PI3K/PTEN-mTORC1 signaling to regulate neurite development (Figures 3-9 and 3-10). In view of other published results indicating an important role of protein synthesis in neurite growth (Jaworski et al., 2005; Kumar et al., 2005), these data are compatible with the notion of Nedd4-1 mRNA as a major target of the local translational machinery in growing neurites. Importantly, accumulating evidences indicate the crucial roles of local protein synthesis in various aspects of normal neuronal development and function, such as axon guidance, dendritic spine formation, and synaptic plasticity (reviewed by Holt and Schuman, 2013; Jung et al., 2012; Sutton and Schuman, 2006). The notion that Nedd4-1 expression may be regulated by the neuronal local translational machinery, therefore, indicates that Nedd4-1 may have important functions in other aspects during neuronal development in addition to the neurite growth regulation.

\subsubsection{Possible Roles of Nedd4-1 in Insulin-Like Growth Factor-1 (IGF- 1) Signaling}

Cortical neurons of the igf1 KO mouse show a decreased length and complexity of dendrites, indicating a role of IGF-1 in promoting dendritic development (Cheng et al., 2003). On the other hand, multiple publications have reported a role of Nedd4-1 in regulating IGF-1 dependent signaling 
(Cao et al., 2008; Monami et al., 2008; Shi et al., 2014), which may account for the reduced outgrowth of neurites seen in the Nedd4-1 KO neurons (Kawabe et al., 2010). However, the physiological relevance of these findings remains unclear and controversial. For instance, Nedd4-1 KO MEFs show a downregulation of surface-expressed IGF-1 receptor (IGF-1R) and reduced IGF-1 signaling (Cao et al., 2008), whereas RNAi-mediated KD of endogenous Nedd4-1 in MEFs leads to increased IGF-1-induced cellular responses (Monami et al. 2008). Based on my current finding that Nedd4-1 expression is positively regulated through mTORC1 and other published results, I propose that Nedd4-1 may play a role in regulating IGF-1 signaling.

In the study by Monami et al (2008), the authors concluded that Nedd41 interacts with growth factor receptor bound protein 10 (Grb10), an adaptor protein that binds to IGF-1R, and that the formation of a Nedd4-1/Grb10/IGF1R complex facilitates monoubiquitination of surface-expressed IGF-1Rs by Nedd4-1, leading to the internalization of IGF-1Rs. This model is consistent with the finding that KD of Grb10 causes upregulation of IGF-1 signaling (Dufresne and Smith, 2005). However, KD of Grb10 also increases IGF-1R surface expression in the absence of Nedd4-1 (Cao et al., 2008), indicating that Grb10 can negatively regulate IGF-1 signaling independent of Nedd4-1.

While in the present study I found that Nedd4-1 mRNA is a target of mTORC1-dependent signaling during translation, recently published studies revealed that Grb10 is a direct phosphorylation substrate of mTORC1. Phosphorylation of Grb10 by mTORC1 increases Grb10 protein stability, leading to a Grb10-mediated negative feed-back loop to downregulate IGF-1 signaling (Hsu et al., 2011; Yu et al., 2011). Of note, the phosphorylation sites of Grb10 targeted by mTORC1 (S501 and S503) are located just next to the Nedd4-1 binding region (Huang and Szebenyi, 2010). This raises the possibilities that phosphorylation of Grb10 by mTORC1 may interfere with its binding to Nedd4-1, and that the binding of Grb10 to Nedd4-1 may prevent Grb10 from being phosphorylated by mTORC1. In accordance with this notion, Grb10 expression is upregulated upon Nedd4-1 downregulation, 
although Nedd4-1 does not ubiquitinate Grb10 or target it for degradation (Cao et al., 2008; Morrione et al., 1999). Taken together, in Nedd4-1 KO cells there may be an increased level of phosphorylated and stabilized Grb10, leading to downregulation of IGF-1 signaling in a Nedd4-1 independent manner, whereas Nedd4-1 could bind to the non-phosphorylated form of Grb10 to mediate the monoubiquitination and internalization of IGF-1Rs (Figure 4-1). The actual mechanism by which Nedd4-1 levels affect the

A

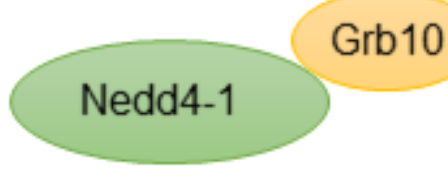

$\square \quad \square$ IGF-1 Signaling

B

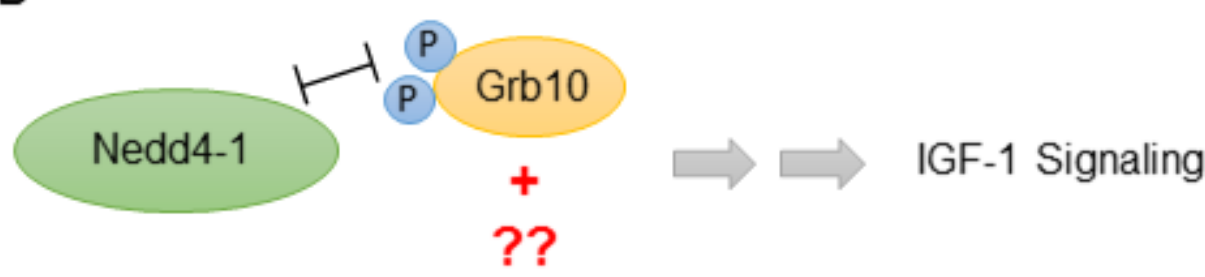

Grb10 phosphorylation by mTORC1, however, remains to be determined.

Figure 4-1. Model of Regulation of IGF-1 Signaling by Nedd4-1 and Grb10

(A) Nedd4-1 interacts with the adaptor protein Grb10, enabling Nedd4-1 to mediate the monoubiquitination and internalization of IGF-1Rs, which further leads to decreased IGF-1 signaling. (B) Binding of Nedd4-1 to Grb10 may hinder the phosphorylation of Grb10 by mTORC1. Phosphorylation of Grb10, on the other hand, may also interfere with Grb10 binding to Nedd4-1. Grb10 can downregulate IGF-1 signaling independently of Nedd4-1, but may cooperate with some yet unknown proteins for this purpose.

\subsection{Opposing Roles of Usp9x and WWP1/WWP2 in the Development of Mammalian Neurons}

In the present study, I identified the deubiquitinase Usp9x as a binding partner of WWP1/WWP2 (Figures 3-13 and 3-14). Usp9x is highly expressed 
in the mammalian CNS and has been shown to deubiquitinate several molecules involved in neurodevelopmental signaling, such as $\beta$-catenin in Wnt signaling (Taya et al., 1999) and Smad4 in TGF- $\beta$ signaling (Xie et al., 2013). Usp9x also interacts with doublecortin, a microtubule-associated protein involved in neuronal migration (Friocourt et al., 2005). These studies indicate that Usp9x may have important functions in the regulation of multiple stages of mammalian neuronal development. Accordingly, conditional KO of Usp9x in mouse neural progenitor cells (Nestin-Cre;Usp9x ${ }^{f / f}$ ) causes perinatal lethality, disrupted cortical architecture, and impaired axon morphogenesis (Homan et al., 2014).

In addition, it has been reported that the C-terminus of Usp9x interacts with the second WW domain of Itch and Smurf1, two other E3 ligases in the Nedd4 superfamily (Figure 1-4). Usp9x inhibits the autoubiquitination of Itch and Smurf1, leading to increased stability and activity of the ligases. On the other hand, Usp9x is not a direct substrate of Itch or Smurf1 (Mouchantaf et al., 2006; Xie et al., 2013). Given the structural similarity between the WW domains of Itch, Smurf1, WWP1, and WWP2 (Figure 1-4), Usp9x may play a similar role in counteracting the activity of WWP1/WWP2 as it does with Itch and Smurf1. Of note, Usp9x has been identified as the deubiquitinase for Nuak1, an AMP-activated protein kinase (AMPK)-related kinase that acts downstream of LKB1 to regulate axonal terminal branching in mouse cortical neurons (Courchet et al., 2013). Deubiquitination of Nuak1 by Usp9x inhibits LKB1-mediated Nuak1 phosphorylation and activation in non-neuronal cells (Al-Hakim et al., 2008). Interestingly, in an in vitro binding assay I found that WWP1 and WWP2 also interact with Nuak1 and Nuak2, the closest homologue of Nuak1 in mammals (Figure 3-14). These results are compatible with the notion that although WWP1/WWP2 do not ubiquitinate Usp9x (Mouchantaf et al., 2006; Xie et al., 2013), they may counteract the functions of Usp9x by ubiquitinating an effector protein that is also targeted by Usp9x, such as Nuak1 and Nuak2. This model (Figure 4-2) also indicates that WWP1/WWP2 may play important roles in multiple processes of neuronal development. The binding between Usp9x and WWP1/WWP2, in 
addition, may operate to bring the deubiquitinase and the ligase to the same intracellular location in order to efficiently fine-tune a highly dynamic cellular process such as axon morphogenesis.

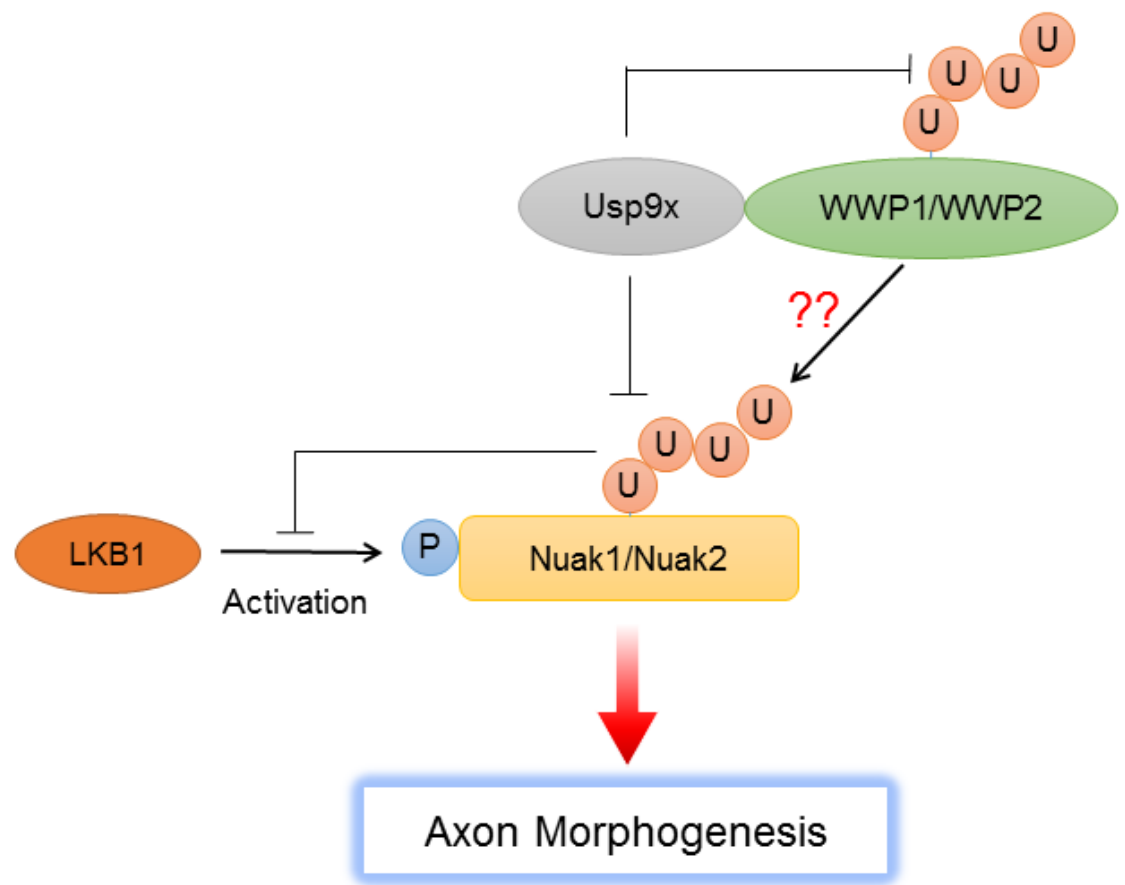

Figure 4-2. Model of Opposing Roles of Usp9x and WWP1/WWP2 in Axon Morphogenesis

LKB1 mediates the phosphorylation and activation of Nuak1/Nuak2, and this regulation is important for axon morphogenesis. Ubiquitination of Nuak1/Nuak2 inhibits their phosphorylation and activation mediated by LKB1, and Usp9x removes such inhibition by deubiquitinating Nuak1/Nuak2. Usp9x is a binding partner of WWP1/WWP2 and may lead to increased stability and activity of WWP1/WWP2 by inhibiting the autoubiquitination of the ligases. WWP1/WWP2 interact with Nuak1 and Nuak2 and may mediate their ubiquitination, counteracting the function of Usp9x in axon morphogenesis.

\subsection{Regulation of Cdk5 by WWP1/WWP2-Mediated Ubiquitination}

Cdk5 is a unique member in the Cdk family with important roles in the CNS. Misregulation of Cdk5 leads to defects in neuronal development and multiple neuronal diseases (reviewed by Dhavan and Tsai, 2001; Su and 
Tsai, 2011). In keeping with its prominent and diverse functions, Cdk5 is tightly regulated at multiple levels, including posttranslational modifications of Cdk5. Here, I identified WWP1 and WWP2 as E3 ligases that mediate K63linked polyubiquitination of Cdk5 (Figures 3-15, 3-16, and 3-17). This type of ubiquitination does not target Cdk5 for proteosomal degradation but is likely involved in the regulation of other functional aspects of Cdk5, such as kinase activity or intracellular localization of Cdk5.

Cdk5 is regulated by various posttranslational modifications. Phosphorylation of Cdk5 at tyrosine 15 (Y15) by c-Abl increases the kinase activity of Cdk5, and this phosphorylation can be facilitated upon binding of Cdk5 with Cable, an adaptor protein to c-Abl (Zukerberg et al., 2000). Cdk5 is also phosphorylated at Y15 by Fyn, a member of the Src family of nonreceptor tyrosine kinases that are associated with neuropilin-1 and plexinA1/A2, receptors for Semaphorin-3A (Sema-3A). This pathway, in which phosphorylation of Cdk5 at Y15 by Fyn upon Sema-3A stimulation, is important for the Sema-3A induced growth cone collapse and the dendriteaxon orientation of mouse cortical neurons (Sasaki et al., 2002). Moreover, one report showed that Cdk5 is S-nitrosylated at Cysteine 83, and such Snitrosylation inhibits the Cdk5 kinase activity and the associated neurite outgrowth during neuronal development (Zhang et al., 2010). Ubiquitination of Cdk5, on the other hand, has only been reported to be mediated by the E3 ligases cdh1-APC, leading to proteosomal degradation of Cdk5 (Zhang et al., 2012). In the present study, I found that Cdk5 is polyubiquitinated by WWP1/WWP2 via K63-linked chains for purposes independent of proteasomal degradation (Figures 3-15 and 3-17). Such ubiquitination of Cdk5 may be involved in the regulation of Cdk5 activity, which is similar to the phosphorylation or S-nitrosylation of Cdk5. The issue of which lysine residue in Cdk5 is ubiquitinated by WWP1/WWP2, however, remains to be resolved.

Activation of Cdk5 is dependent upon binding to its activators p35 and p39, which have partially complementary temporal and spatial expression 
patterns in the CNS (Wu et al., 2000; Zheng et al., 1998). As the main activator of Cdk5 in the cerebral cortex and hippocampus, p35 is a shortlived protein with a half-life of only 20 to 30 minutes. Once p35 binds to Cdk5, it is phosphorylated by Cdk5, resulting in rapid ubiquitination and degradation of p35 (Patrick et al., 1998). The unstable nature of the p35 protein underscores the importance of regulating $p 35$ expression levels. It has been reported that application of the extracellular glycoprotein, laminin, to cultured neurons or the SH-SY5Y neuroblastoma cell line results in increased p35 mRNA and protein levels as well as the corresponding Cdk5 activity (Li et al., 2000; Paglini et al., 1998). In addition, induction of the transcription factor early growth response 1 (EGR1) by the extracellular-signal-regulated kinase (ERK) cascade also leads to the upregulation of p35 expression. Importantly, I found that the Cdk5 ubiquitination mediated by WWP1 in vitro is substantially reduced in the presence of high-levels of p25, an N-terminally truncated form of $p 35$. This result indicates that binding with p25 blocks the ubiquitination of Cdk5 by WWP1, or, on the contrary, that ubiquitination of Cdk5 by WWP ligases may interfere with the binding of $\mathrm{p} 25$ to Cdk5 and thus affect Cdk5 activation (Figure 3-17 C).

In view of the fact that K63-linked polyubiquitination of proteins may lead to changes in protein localization (reviewed by Ikeda and Dikic, 2008), the ubiquitination of Cdk5 by WWP1/WWP2 may regulate the cellular localization of Cdk5. Of note, in the N-terminus of p35 there is a myristoylation signal motif that operates to target the p35/Cdk5 complex to the membrane. Accordingly, many of the identified physiological substrates of Cdk5 are located at the cell membrane (reviewed by Su and Tsai, 2011). The calpain-mediated conversion of p35 to p25, an N-terminally truncated form of p35, leads to the cytosolic localization of activated Cdk5 and aberrantly increased Cdk5 activity, because of the loss of the myristoylation signal motif on p25 and the prolonged half-life of p25 as compared to p35 (Patrick et al., 1999). In addition, the nuclear localization of Cdk5 appears to play important roles for neuronal protection upon stress or insult, since loss 
of the nuclear Cdk5 has been linked with the cell-cycle re-entry of postmitotic neurons, leading to neuronal cell death (Zhang et al., 2008).

In summary, the localization and activity of Cdk5 are vital for the proper functions of various cellular processes in the central nervous system and should be tightly regulated. Ubiquitination of Cdk5 by the WWP E3 ligases, WWP1 and WWP2, may contribute to the regulation of Cdk5 and thereby have important roles in neuronal development.

\subsection{Conclusions and Outlook}

In the classical view of protein ubiquitination, substrate proteins are conjugated with K48-linked polyubiquitin chains and targeted for proteosomal degradation. Recent progress, however, indicates that target proteins may undergo polyubiquitination that is conjugated via the other six lysine residues of the ubiquitin moiety. This sophisticated nature of protein ubiquitination contributes another layer of complexity and results in obstacles to study its physiological roles. Given that the ubiquitin E3 ligases govern the substrate specificity, studies focusing on the functions and regulation of a certain E3 ligase offer an entry point to understand the possible roles of protein ubiquitination in defined cellular processes. The present work has focused on the characterization of the E3 ligases Nedd4-1, Nedd4-2, WWP1, and WWP2 in the regulation of neuronal development. These ligases are members of the HECT-type Nedd4 superfamily that have been shown to preferentially conjugate substrate proteins with monoubiquitin or K63-linked polyubiquitin for non-proteolytic purposes (Kamadurai et al., 2013; Kim and Huibregtse, 2009; Polo et al., 2002).

In the first part of the study, I investigated the roles of Nedd4-1 and Nedd4-2 in axon morphogenesis of mammalian CNS neurons, and I found that Nedd4-1/Nedd4-2 promote axon outgrowth in mouse hippocampal and cortical neurons (Figures 3-1, 3-3, and 3-4). While xNedd4 was shown to promote axon growth in Xenopus laevis retinal ganglion cells by targeting 
PTEN for proteosomal degradation (Drinjakovic et al., 2010), I found no evidence of misregulation of PTEN that could contribute to the axonal growth defects observed in Nedd4-1;Nedd4-2 KO neurons. These results argue against the belief that PTEN is targeted by Nedd4-1 or Nedd4-2 in general cellular processes. In contrast, I found that PTEN negatively regulates Nedd4-1 expression at the translational level by antagonizing PI3K-mTORC1 signaling, and that this regulation is important for neurite outgrowth. Of note, these findings are in accordance with the notion that Nedd4-1 mRNAs are targeted by the translational machinery in growing neurites and raise the possibility that Nedd4-1 expression can be spatially and temporally controlled in the cells. Given that accumulating evidence indicates the importance of local mRNA translation in neuronal axon guidance and synaptic plasticity, additional studies on how Nedd4-1 expression is regulated in response to extracellular signals with higher spatiotemporal resolution may give further insights into the roles of Nedd4-1 in axonal guidance and synaptic plasticity. Moreover, independent regulatory programs driven by either PI3K/PTENmTORC1 or Nedd4-1 may interact and regulate the same cellular process. For instance, mTORC1 promotes the translation of Nedd4-1 as well as the phosphorylation of Grb10, a Nedd4-1 binding partner that may regulate IGF1 signaling in a Nedd4-1-dependent or -independent manner (Figure 4-1). Future studies should address if and how Grb10 phosphorylation by mTORC1 is affected by Nedd4-1 expression.

In the second part of the study, I identified Usp9x, Nuak1, and Nuak2 as binding partners of WWP1/WWP2, and Cdk5 as a physiological substrate of WWP1/WWP2. Based on these results and other published data, WWP1/WWP2 may have important roles in axon morphogenesis by ubiquitinating Nuak1/Nuak2, counteracting the function of Usp9x. In addition, ubiquitination of Cdk5 by WWP1/WWP2 may change Cdk5 activity or localization, and thereby affect various cellular processes given the vital roles of Cdk5 in neuronal development and functions. Future studies on the consequences of WWP1/WWP2 deletion in neurons may shed light on how 
WWP1/WWP2 regulate Usp9x, Nuak1, Nuak2, and Cdk5 via direct interaction or ubiquitination. 


\section{References}

Ahmed, S.F., Deb, S., Paul, I., Chatterjee, A., Mandal, T., Chatterjee, U., and Ghosh, M.K. (2012). The chaperone-assisted E3 ligase C terminus of Hsc70-interacting protein (CHIP) targets PTEN for proteasomal degradation. J. Biol. Chem. 287, 15996-16006.

Ahn, Y., Hwang, C.Y., Lee, S.-R., Kwon, K.-S., and Lee, C. (2008). The tumour suppressor PTEN mediates a negative regulation of the E3 ubiquitin-protein ligase Nedd4. Biochem. J. 412, 331-338.

Amodio, N., Scrima, M., Palaia, L., Salman, A.N., Quintiero, A., Franco, R., Botti, G., Pirozzi, P., Rocco, G., De Rosa, N., et al. (2010). Oncogenic role of the E3 ubiquitin ligase NEDD4-1, a PTEN negative regulator, in non-small-cell lung carcinomas. Am. J. Pathol. 177, 2622-2634.

Arimura, N., and Kaibuchi, K. (2007). Neuronal polarity: from extracellular signals to intracellular mechanisms. Nat. Rev. Neurosci. 8, 194-205.

Bagni, C., and Greenough, W.T. (2005). From mRNP trafficking to spine dysmorphogenesis: the roots of fragile X syndrome. Nat. Rev. Neurosci. 6, 376-387.

Barnes, A.P., and Polleux, F. (2009). Establishment of axon-dendrite polarity in developing neurons. Annu. Rev. Neurosci. 32, 347-381.

Boase, N.A., Rychkov, G.Y., Townley, S.L., Dinudom, A., Candi, E., Voss, A.K., Tsoutsman, T., Semsarian, C., Melino, G., Koentgen, F., et al. (2011). Respiratory distress and perinatal lethality in Nedd4-2-deficient mice. Nat. Commun. 2, 287.

Brose, N. (1999). Synaptic cell adhesion proteins and synaptogenesis in the mammalian central nervous system. Naturwissenschaften $86,516-524$.

Brown, M., Jacobs, T., Eickholt, B., Ferrari, G., Teo, M., Monfries, C., Qi, R.Z., Leung, T., Lim, L., and Hall, C. (2004). a2-chimaerin, cyclin-dependent kinase $5 / \mathrm{p} 35$, and its target collapsin response mediator protein-2 are essential components in semaphorin 3A-induced growth-cone collapse. J. Neurosci. 24, 8994-9004.

Cao, X.R., Lill, N.L., Boase, N., Shi, P.P., Croucher, D.R., Shan, H., Qu, J., Sweezer, E.M., Place, T., Kirby, P.A., et al. (2008). Nedd4 controls animal growth by regulating IGF-1 signaling. Sci Signal 1 , ra5.

Chae, T., Kwon, Y.T., Bronson, R., Dikkes, P., Li, E., and Tsai, L.-H. (1997). Mice lacking p35, a neuronal specific activator of $\mathrm{Cdk} 5$, display cortical lamination defects, seizures, and adult lethality. Neuron 18, 29-42.

Chen, C., Sun, X., Guo, P., Dong, X.-Y., Sethi, P., Zhou, W., Zhou, Z., Petros, J., Frierson, H.F., Vessella, R.L., et al. (2006). Ubiquitin E3 ligase WWP1 as an oncogenic factor in human prostate cancer. Oncogene 26, 2386-2394. 
Chen, C., Zhou, Z., Sheehan, C.E., Slodkowska, E., Sheehan, C.B., Boguniewicz, A., and Ross, J.S. (2009). Overexpression of WWP1 is associated with the estrogen receptor and insulin-like growth factor receptor 1 in breast carcinoma. Int. J. Cancer 124, 2829-2836.

Cheng, P., and Poo, M. (2012). Early events in axon/dendrite polarization. Annu. Rev. Neurosci. 35, 181-201.

Cheng, C.M., Mervis, R.F., Niu, S.-L., Salem, N., Witters, L.A., Tseng, V., Reinhardt, R., and Bondy, C.A. (2003). Insulin-like growth factor 1 is essential for normal dendritic growth. J. Neurosci. Res. 73, 1-9.

Cheung, Z.H., Chin, W.H., Chen, Y., Ng, Y.P., and Ip, N.Y. (2007). Cdk5 is involved in BDNF-stimulated dendritic growth in hippocampal neurons. PLoS Biol. 5 .

Ch'ng, Q., Sieburth, D., and Kaplan, J.M. (2008). Profiling synaptic proteins identifies regulators of insulin secretion and lifespan. PLoS Genet 4, e1000283.

Chow, D.K., Groszer, M., Pribadi, M., Machniki, M., Carmichael, S.T., Liu, X., and Trachtenberg, J.T. (2009). Laminar and compartmental regulation of dendritic growth in mature cortex. Nat Neurosci 12, 116-118.

Christie, K.J., Martinez, J.A., and Zochodne, D.W. (2012). Disruption of E3 ligase NEDD4 in peripheral neurons interrupts axon outgrowth: Linkage to PTEN. Mol. Cell. Neurosci. 50, 179-192.

Chung, S., Nakashima, M., Zembutsu, H., and Nakamura, Y. (2011). Possible involvement of NEDD4 in keloid formation; its critical role in fibroblast proliferation and collagen production. Proc. Jpn. Acad. Ser. B 87, 563-573.

Courchet, J., Lewis Jr., T.L., Lee, S., Courchet, V., Liou, D.-Y., Aizawa, S., and Polleux, F. (2013). Terminal Axon Branching Is Regulated by the LKB1-NUAK1 Kinase Pathway via Presynaptic Mitochondrial Capture. Cell 153, 1510-1525.

Dhavan, R., and Tsai, L.-H. (2001). A decade of Cdk5. Nat. Rev. Mol. Cell Biol. 2, 749-759.

DiAntonio, A., and Hicke, L. (2004). Ubiquitin-dependent regulation of the synapse. Annu. Rev. Neurosci. 27, 223-246.

Dierssen, M., and Ramakers, G.J.A. (2006). Dendritic pathology in mental retardation: from molecular genetics to neurobiology. Genes Brain Behav. 5, 48-60.

Drinjakovic, J., Jung, H., Campbell, D.S., Strochlic, L., Dwivedy, A., and Holt, C.E. (2010). E3 ligase Nedd4 promotes axon branching by downregulating PTEN. Neuron $65,341-357$. 
Dufresne, A.M., and Smith, R.J. (2005). The adapter protein GRB10 is an endogenous negative regulator of insulin-like growth factor signaling. Endocrinology 146, 4399-4409.

Fischer, A., Sananbenesi, F., Pang, P.T., Lu, B., and Tsai, L.-H. (2005). Opposing roles of transient and prolonged expression of p25 in synaptic plasticity and hippocampus-dependent memory. Neuron 48, 825-838.

Fouladkou, F., Landry, T., Kawabe, H., Neeb, A., Lu, C., Brose, N., Stambolic, V., and Rotin, D. (2008). The ubiquitin ligase Nedd4-1 is dispensable for the regulation of PTEN stability and localization. Proc. Natl. Acad. Sci. 105, 8585-8590.

Fraser, M.M., Bayazitov, I.T., Zakharenko, S.S., and Baker, S.J. (2008). Phosphatase and tensin homolog, deleted on chromosome 10 deficiency in brain causes defects in synaptic structure, transmission and plasticity, and myelination abnormalities. Neuroscience 151, 476-488.

Friocourt, G., Kappeler, C., Saillour, Y., Fauchereau, F., Rodriguez, M.S., Bahi, N., Vinet, M.-C., Chafey, P., Poirier, K., Taya, S., et al. (2005). Doublecortin interacts with the ubiquitin protease DFFRX, which associates with microtubules in neuronal processes. Mol. Cell. Neurosci. 28, 153-164.

Frotscher, M. (1998). Cajal--Retzius cells, Reelin, and the formation of layers. Curr. Opin. Neurobiol. 8, 570-575.

Garner, C.C., Zhai, R.G., Gundelfinger, E.D., and Ziv, N.E. (2002). Molecular mechanisms of CNS synaptogenesis. Trends Neurosci. 25, 243-250.

Gingras, A.-C., Raught, B., and Sonenberg, N. (1999). eIF4 initiation factors: effectors of mRNA recruitment to ribosomes and regulators of translation. Annu. Rev. Biochem. 68, 913-963.

Goebbels, S., Bormuth, I., Bode, U., Hermanson, O., Schwab, M.H., and Nave, K.A. (2006). Genetic targeting of principal neurons in neocortex and hippocampus of NEX-Cre mice. Genes. N. Y. N 2000 44, 611-621.

Gotz, M., and Huttner, W.B. (2005). The cell biology of neurogenesis. Nat Rev Mol Cell Biol 6, 777-788.

Groszer, M., Erickson, R., Scripture-Adams, D.D., Lesche, R., Trumpp, A., Zack, J.A., Kornblum, H.I., Liu, X., and Wu, H. (2001). Negative regulation of neural stem/progenitor cell proliferation by the Pten tumor suppressor gene in vivo. Science 294, 2186-2189.

Groszer, M., Erickson, R., Scripture-Adams, D.D., Dougherty, J.D., Belle, J.L., Zack, J.A., Geschwind, D.H., Liu, X., Kornblum, H.I., and Wu, H. (2006). PTEN negatively regulates neural stem cell self-renewal by modulating G0-G1 cell cycle entry. Proc. Natl. Acad. Sci. U. S. A. 103, 111-116. 
Guo, H., Qiao, G., Ying, H., Li, Z., Zhao, Y., Liang, Y., Yang, L., Lipkowitz, S., Penninger, J.M., Langdon, W.Y., et al. (2012). E3 ubiquitin ligase Cbl-b regulates Pten via Nedd 4 in T cells independently of its ubiquitin ligase activity. Cell Rep. 1, 472-482.

Gupta, R., Kus, B., Fladd, C., Wasmuth, J., Tonikian, R., Sidhu, S., Krogan, N.J., Parkinson, J., and Rotin, D. (2007). Ubiquitination screen using protein microarrays for comprehensive identification of Rsp5 substrates in yeast. Mol. Syst. Biol. 3, 116.

Al-Hakim, A.K., Zagorska, A., Chapman, L., Deak, M., Peggie, M., and Alessi, D.R. (2008). Control of AMPK-related kinases by USP9X and atypical lys29/lys33-linked polyubiquitin chains. Biochem. J. 411, 249.

Hammond, V.E., Gunnersen, J.M., Goh, C.-P., Low, L.-H., Hyakumura, T., Tang, M.M., Britto, J.M., Putz, U., Howitt, J.A., and Tan, S.-S. (2013). Ndfip1 is required for the development of pyramidal neuron dendrites and spines in the neocortex. Cereb. Cortex.

Herculano-Houzel, S., Mota, B., and Lent, R. (2006). Cellular scaling rules for rodent brains. Proc. Natl. Acad. Sci. 103, 12138-12143.

Hjerpe, R., Aillet, F., Lopitz-Otsoa, F., Lang, V., England, P., and Rodriguez, M.S. (2009). Efficient protection and isolation of ubiquitylated proteins using tandem ubiquitin-binding entities. EMBO Rep. 10, 1250-1258.

Holt, C.E., and Schuman, E.M. (2013). The central dogma decentralized: new perspectives on RNA function and local translation in neurons. Neuron 80, 648-657.

Homan, C.C., Kumar, R., Nguyen, L.S., Haan, E., Raymond, F.L., Abidi, F., Raynaud, M., Schwartz, C.E., Wood, S.A., Gecz, J., et al. (2014). Mutations in USP9X are associated with X-linked intellectual disability and disrupt neuronal cell migration and growth. Am. J. Hum. Genet. 94, 470-478.

Honda, T., Kobayashi, K., Mikoshiba, K., and Nakajima, K. (2011). Regulation of cortical neuron migration by the reelin signaling pathway. Neurochem. Res. 36, 1270-1279.

Howitt, J., Lackovic, J., Low, L.-H., Naguib, A., Macintyre, A., Goh, C.-P., Callaway, J.K., Hammond, V., Thomas, T., Dixon, M., et al. (2012). Ndfip1 regulates nuclear PTEN import in vivo to promote neuronal survival following cerebral ischemia. J. Cell Biol. 196, 29-36.

Hsia, H.-E., Kumar, R., Luca, R., Takeda, M., Courchet, J., Nakashima, J., Wu, S., Goebbels, S., An, W., Eickholt, B.J., et al. (2014). Ubiquitin E3 ligase Nedd4-1 acts as a downstream target of PI3K/PTEN-mTORC1 signaling to promote neurite growth. Proc. Natl. Acad. Sci. 111, 13205-13210.

Hsu, P.P., Kang, S.A., Rameseder, J., Zhang, Y., Ottina, K.A., Lim, D., Peterson, T.R., Choi, Y., Gray, N.S., Yaffe, M.B., et al. (2011). The mTOR-regulated 
phosphoproteome reveals a mechanism of mTORC1-mediated inhibition of growth factor signaling. Science 332, 1317-1322.

Huang, Q., and Szebenyi, D.M.E. (2010). Structural basis for the interaction between the growth factor-binding protein GRB10 and the E3 ubiquitin ligase NEDD4. J. Biol. Chem. 285, 42130-42139.

Humbert, S., Lanier, L.M., and Tsai, L.-H. (2000). Synaptic localization of p39, a neuronal activator of cdk5. Neuroreport July 142000 11, 2213-2216.

Ikeda, F., and Dikic, I. (2008). Atypical ubiquitin chains: new molecular signals. EMBO Rep 9, 536-542.

Jahn, O., Hesse, D., Reinelt, M., and Kratzin, H.D. (2006). Technical innovations for the automated identification of gel-separated proteins by MALDI-TOF mass spectrometry. Anal. Bioanal. Chem. 386, 92-103.

Jan, Y.-N., and Jan, L.Y. (2010). Branching out: mechanisms of dendritic arborization. Nat Rev Neurosci 11, 316-328.

Jaworski, J., Spangler, S., Seeburg, D.P., Hoogenraad, C.C., and Sheng, M. (2005). Control of dendritic arborization by the phosphoinositide-3'-kinase-Akt-mammalian target of rapamycin pathway. J. Neurosci. 25, 11300-11312.

Jiang, H., Guo, W., Liang, X., and Rao, Y. (2005). Both the establishment and the maintenance of neuronal polarity require active mechanisms: critical roles of GSK3 beta and its upstream regulators. Cell 120, 123-135.

Jung, H., Yoon, B.C., and Holt, C.E. (2012). Axonal mRNA localization and local protein synthesis in nervous system assembly, maintenance and repair. Nat. Rev. Neurosci. 13, 445-445.

Kamadurai, H.B., Qiu, Y., Deng, A., Harrison, J.S., MacDonald, C., Actis, M., Rodrigues, P., Miller, D.J., Souphron, J., Lewis, S.M., et al. (2013). Mechanism of ubiquitin ligation and lysine prioritization by a HECT E3. eLife 2, e00828.

Kaufmann, W.E., and Moser, H.W. (2000). Dendritic Anomalies in Disorders Associated with Mental Retardation. Cereb. Cortex 10, 981-991.

Kawabe, H., and Brose, N. (2011). The role of ubiquitylation in nerve cell development. Nat Rev Neurosci 12, 251-268.

Kawabe, H., Neeb, A., Dimova, K., Young Jr., S.M., Takeda, M., Katsurabayashi, S., Mitkovski, M., Malakhova, O.A., Zhang, D.-E., Umikawa, M., et al. (2010). Regulation of Rap2A by the ubiquitin ligase Nedd4-1 controls neurite development. Neuron 65, 358-372.

Kim, H.C., and Huibregtse, J.M. (2009). Polyubiquitination by HECT E3s and the determinants of chain type specificity. Mol. Cell. Biol. 29, 3307-3318. 
Kim, W., Bennett, E.J., Huttlin, E.L., Guo, A., Li, J., Possemato, A., Sowa, M.E., Rad, R., Rush, J., Comb, M.J., et al. (2011). Systematic and quantitative assessment of the ubiquitin-modified proteome. Mol. Cell 44, 325-340.

Kimura, T., Kawabe, H., Jiang, C., Zhang, W., Xiang, Y.-Y., Lu, C., Salter, M.W., Brose, N., Lu, W.-Y., and Rotin, D. (2011). Deletion of the ubiquitin ligase Nedd4L in lung epithelia causes cystic fibrosis-like disease. Proc. Natl. Acad. Sci. 108, 32163221 .

Kishi, M., Pan, Y.A., Crump, J.G., and Sanes, J.R. (2005). Mammalian SAD kinases are required for neuronal polarization. Science 307, 929-932.

Ko, J., Humbert, S., Bronson, R.T., Takahashi, S., Kulkarni, A.B., Li, E., and Tsai, L.-H. (2001). p35 and p39 are essential for cyclin-dependent kinase 5 function during neurodevelopment. J. Neurosci. 21, 6758-6771.

Komander, D., and Rape, M. (2012). The ubiquitin code. Annu. Rev. Biochem. 81, 203-229.

Krüger, M., Moser, M., Ussar, S., Thievessen, I., Luber, C.A., Forner, F., Schmidt, S., Zanivan, S., Fässler, R., and Mann, M. (2008). SILAC Mouse for quantitative proteomics uncovers kindlin-3 as an essential factor for red blood cell function. Cell $134,353-364$.

Kumar, V., Zhang, M.-X., Swank, M.W., Kunz, J., and Wu, G.-Y. (2005). Regulation of dendritic morphogenesis by Ras-PI3K-Akt-mTOR and Ras-MAPK signaling pathways. J. Neurosci. 25, 11288-11299.

Kus, B., Gajadhar, A., Stanger, K., Cho, R., Sun, W., Rouleau, N., Lee, T., Chan, D., Wolting, C., Edwards, A., et al. (2005). A high throughput screen to identify substrates for the ubiquitin ligase Rsp5. J. Biol. Chem. 280, 29470-29478.

Kwak, Y.-D., Wang, B., Pan, W., Xu, H., Jiang, X., and Liao, F.-F. (2010). Functional interaction of phosphatase and tensin homologue (PTEN) with the E3 ligase NEDD4-1 during neuronal response to zinc. J. Biol. Chem. 285, 9847-9857.

Kwon, C.-H., Zhu, X., Zhang, J., Knoop, L.L., Tharp, R., Smeyne, R.J., Eberhart, C.G., Burger, P.C., and Baker, S.J. (2001). Pten regulates neuronal soma size: a mouse model of Lhermitte-Duclos disease. Nat. Genet. 29, 404-411.

Kwon, C.-H., Luikart, B.W., Powell, C.M., Zhou, J., Matheny, S.A., Zhang, W., Li, Y., Baker, S.J., and Parada, L.F. (2006). PTEN regulates neuronal arborization and social interaction in mice. Neuron 50,377-388.

Lambert de Rouvroit, C., and Goffinet, A.M. (2001). Neuronal migration. Mech. Dev. 105, 47-56.

Lew, J., Beaudette, K., Litwin, C.M., and Wang, J.H. (1992). Purification and characterization of a novel proline-directed protein kinase from bovine brain. J. Biol. Chem. 267, 13383-13390. 
Li, B.-S., Zhang, L., Gu, J., Amin, N.D., and Pant, H.C. (2000). Integrin $\alpha 1 \beta 1-$ mediated activation of cyclin-dependent kinase 5 activity is involved in neurite outgrowth and human neurofilament protein $\mathrm{H}$ Lys-Ser-Pro tail domain phosphorylation. J. Neurosci. 20, 6055-6062.

Li, Y., Zhou, Z., Alimandi, M., and Chen, C. (2009). WW domain containing E3 ubiquitin protein ligase 1 targets the full-length ErbB4 for ubiquitin-mediated degradation in breast cancer. Oncogene 28, 2948-2958.

Luikart, B.W., Schnell, E., Washburn, E.K., Bensen, A.L., Tovar, K.R., and Westbrook, G.L. (2011). PTEN knockdown in vivo increases excitatory drive onto dentate granule cells. J. Neurosci. 31, 4345-4354.

Maddika, S., Kavela, S., Rani, N., Palicharla, V.R., Pokorny, J.L., Sarkaria, J.N., and Chen, J. (2011). WWP2 is an E3 ubiquitin ligase for PTEN. Nat. Cell Biol. 13, 728733 .

Meyerson, M., Enders, G.H., Wu, C.L., Su, L.K., Gorka, C., Nelson, C., Harlow, E., and Tsai, L.H. (1992). A family of human cdc2-related protein kinases. EMBO J. 11, 2909-2917.

Monami, G., Emiliozzi, V., and Morrione, A. (2008). Grb10/Nedd4-mediated multiubiquitination of the insulin-like growth factor receptor regulates receptor internalization. J. Cell. Physiol. 216, 426-437.

Morabito, M.A., Sheng, M., and Tsai, L.-H. (2004). Cyclin-dependent kinase 5 phosphorylates the N-terminal domain of the postsynaptic density protein PSD-95 in neurons. J. Neurosci. 24, 865-876.

Morrione, A., Plant, P., Valentinis, B., Staub, O., Kumar, S., Rotin, D., and Baserga, R. (1999). mGrb10 interacts with Nedd4. J. Biol. Chem. 274, 24094-24099.

Mouchantaf, R., Azakir, B.A., McPherson, P.S., Millard, S.M., Wood, S.A., and Angers, A. (2006). The ubiquitin ligase itch is auto-ubiquitylated in vivo and in vitro but is protected from degradation by interacting with the deubiquitylating enzyme FAM/USP9X. J. Biol. Chem. 281, 38738-38747.

Mund, T., and Pelham, H.R.B. (2009). Control of the activity of WW-HECT domain E3 ubiquitin ligases by NDFIP proteins. EMBO Rep. 10, 501-507.

Nguyen Huu, N., Ryder, W., Zeps, N., Flasza, M., Chiu, M., Hanby, A., Poulsom, R., Clarke, R., and Baron, M. (2008). Tumour-promoting activity of altered WWP1 expression in breast cancer and its utility as a prognostic indicator. J. Pathol. 216, 93-102.

Niethammer, M., Smith, D.S., Ayala, R., Peng, J., Ko, J., Lee, M.-S., Morabito, M., and Tsai, L.-H. (2000). NUDEL is a novel Cdk5 substrate that associates with LIS1 and cytoplasmic dynein. Neuron 28,697-711. 
Nikolic, M., Dudek, H., Kwon, Y.T., Ramos, Y.F., and Tsai, L.H. (1996). The $\mathrm{cdk} 5 / \mathrm{p} 35$ kinase is essential for neurite outgrowth during neuronal differentiation. Genes Dev. 10, 816-825.

O'Donnell, M., Chance, R.K., and Bashaw, G.J. (2009). Axon growth and guidance: receptor regulation and signal transduction. Annu. Rev. Neurosci. 32, 383-412.

Ohshima, T., Ward, J.M., Huh, C.G., Longenecker, G., Veeranna, Pant, H.C., Brady, R.O., Martin, L.J., and Kulkarni, A.B. (1996). Targeted disruption of the cyclindependent kinase 5 gene results in abnormal corticogenesis, neuronal pathology and perinatal death. Proc. Natl. Acad. Sci. 93, 11173-11178.

Ohshima, T., Hirasawa, M., Tabata, H., Mutoh, T., Adachi, T., Suzuki, H., Saruta, K., Iwasato, T., Itohara, S., Hashimoto, M., et al. (2007). Cdk5 is required for multipolar-to-bipolar transition during radial neuronal migration and proper dendrite development of pyramidal neurons in the cerebral cortex. Development 134, 22732282.

Paglini, G., Pigino, G., Kunda, P., Morfini, G., Maccioni, R., Quiroga, S., Ferreira, A., and Cáceres, A. (1998). Evidence for the participation of the neuron-specific CDK5 activator p35 during laminin-enhanced axonal growth. J. Neurosci. 18, 98589869.

Pardo, C.A., and Eberhart, C.G. (2007). The neurobiology of autism. Brain Pathol. $17,434-447$.

Parrish, J.Z., Emoto, K., Kim, M.D., and Jan, Y.N. (2007). Mechanisms that Regulate Establishment, Maintenance, and Remodeling of Dendritic Fields. Annu. Rev. Neurosci. 30, 399-423.

Patrick, G.N., Zhou, P., Kwon, Y.T., Howley, P.M., and Tsai, L.-H. (1998). p35, the neuronal-specific activator of cyclin-dependent kinase $5(\mathrm{Cdk} 5)$ is degraded by the ubiquitin-proteasome pathway. J. Biol. Chem. 273, 24057-24064.

Patrick, G.N., Zukerberg, L., Nikolic, M., de la Monte, S., Dikkes, P., and Tsai, L.H. (1999). Conversion of p35 to p25 deregulates Cdk5 activity and promotes neurodegeneration. Nature 402, 615-622.

Peng, J., Schwartz, D., Elias, J.E., Thoreen, C.C., Cheng, D., Marsischky, G., Roelofs, J., Finley, D., and Gygi, S.P. (2003). A proteomics approach to understanding protein ubiquitination. Nat. Biotechnol. 21, 921-926.

Pirozzi, G., McConnell, S.J., Uveges, A.J., Carter, J.M., Sparks, A.B., Kay, B.K., and Fowlkes, D.M. (1997). Identification of novel human WW domain-containing proteins by cloning of ligand targets. J. Biol. Chem. 272, 14611-14616.

Polo, S., Sigismund, S., Faretta, M., Guidi, M., Capua, M.R., Bossi, G., Chen, H., De Camilli, P., and Di Fiore, P.P. (2002). A single motif responsible for ubiquitin recognition and monoubiquitination in endocytic proteins. Nature 416, 451-455. 
Puram, S.V., and Bonni, A. (2013). Cell-intrinsic drivers of dendrite morphogenesis. Development 140, 4657-4671.

Putz, U., Howitt, J., Doan, A., Goh, C.-P., Low, L.-H., Silke, J., and Tan, S.-S. (2012). The tumor suppressor PTEN is exported in exosomes and has phosphatase activity in recipient cells. Sci. Signal. 5 , ra70.

Reumann, S., Babujee, L., Ma, C., Wienkoop, S., Siemsen, T., Antonicelli, G.E., Rasche, N., Lüder, F., Weckwerth, W., and Jahn, O. (2007). Proteome analysis of arabidopsis leaf peroxisomes reveals novel targeting peptides, metabolic pathways, and defense mechanisms. Plant Cell Online 19, 3170-3193.

Rotin, D., and Kumar, S. (2009). Physiological functions of the HECT family of ubiquitin ligases. Nat Rev Mol Cell Biol 10, 398-409.

Rui, Y., Myers, K.R., Yu, K., Wise, A., De Blas, A.L., Hartzell, H.C., and Zheng, J.Q. (2013). Activity-dependent regulation of dendritic growth and maintenance by glycogen synthase kinase $3 \beta$. Nat. Commun. 4.

Samuels, B.A., Hsueh, Y.-P., Shu, T., Liang, H., Tseng, H.-C., Hong, C.-J., Su, S.C., Volker, J., Neve, R.L., Yue, D.T., et al. (2007). Cdk5 promotes synaptogenesis by regulating the subcellular distribution of the MAGUK family member CASK. Neuron 56, 823-837.

Sasaki, S., Shionoya, A., Ishida, M., Gambello, M.J., Yingling, J., Wynshaw-Boris, A., and Hirotsune, S. (2000). A LIS1/NUDEL/cytoplasmic dynein heavy chain complex in the developing and adult nervous system. Neuron 28, 681-696.

Sasaki, Y., Cheng, C., Uchida, Y., Nakajima, O., Ohshima, T., Yagi, T., Taniguchi, M., Nakayama, T., Kishida, R., Kudo, Y., et al. (2002). Fyn and Cdk5 mediate semaphorin-3A signaling, which is involved in regulation of dendrite orientation in cerebral cortex. Neuron 35, 907-920.

Shi, Y., Wang, J., Chandarlapaty, S., Cross, J., Thompson, C., Rosen, N., and Jiang, X. (2014). PTEN is a protein tyrosine phosphatase for IRS1. Nat. Struct. Mol. Biol. $21,522-527$.

Shu, L., Zhang, H., Boyce, B.F., and Xing, L. (2013). Ubiquitin E3 ligase WWP1 negatively regulates osteoblast function by inhibiting osteoblast differentiation and migration. J. Bone Miner. Res. 28, 1925-1935.

Siddiqui, T.J., and Craig, A.M. (2011). Synaptic organizing complexes. Curr. Opin. Neurobiol. 21, 132-143.

Sieburth, D., Ch'ng, Q., Dybbs, M., Tavazoie, M., Kennedy, S., Wang, D., Dupuy, D., Rual, J.-F., Hill, D.E., Vidal, M., et al. (2005). Systematic analysis of genes required for synapse structure and function. Nature 436, 510-517.

Song, M.S., Salmena, L., and Pandolfi, P.P. (2012). The functions and regulation of the PTEN tumour suppressor. Nat. Rev. Mol. Cell Biol. 13, 283-296. 
Su, S.C., and Tsai, L.-H. (2011). Cyclin-Dependent Kinases in Brain Development and Disease. Annu. Rev. Cell Dev. Biol. 27, 465-491.

Sutton, M.A., and Schuman, E.M. (2006). Dendritic protein synthesis, synaptic plasticity, and memory. Cell 127, 49-58.

Takemoto-Kimura, S., Suzuki, K., Kamijo, S., Ageta-Ishihara, N., Fujii, H., Okuno, H., and Bito, H. (2010). Differential roles for CaM kinases in mediating excitationmorphogenesis coupling during formation and maturation of neuronal circuits. Eur. J. Neurosci. 32, 224-230.

Tanaka, T., Serneo, F.F., Tseng, H.-C., Kulkarni, A.B., Tsai, L.-H., and Gleeson, J.G. (2004). Cdk5 phosphorylation of doublecortin Ser297 regulates its effect on neuronal migration. Neuron 41, 215-227.

Tang, D., Yeung, J., Lee, K.Y., Matsushita, M., Matsui, H., Tomizawa, K., Hatase, O., and Wang, J.H. (1995). An isoform of the neuronal cyclin-dependent kinase 5 (Cdk5) activator. J. Biol. Chem. 270, 26897-26903.

Taya, S., Yamamoto, T., Kanai-Azuma, M., Wood, S.A., and Kaibuchi, K. (1999). The deubiquitinating enzyme Fam interacts with and stabilizes $\beta$-catenin. Genes Cells 4, 757-767.

Van Themsche, C., Leblanc, V., Parent, S., and Asselin, E. (2009). X-linked inhibitor of apoptosis protein (XIAP) regulates PTEN ubiquitination, content, and compartmentalization. J. Biol. Chem. 284, 20462-20466.

Thoreen, C.C., Chantranupong, L., Keys, H.R., Wang, T., Gray, N.S., and Sabatini, D.M. (2012). A unifying model for mTORC1-mediated regulation of mRNA translation. Nature 485, 109-113.

Tojima, T., Hines, J.H., Henley, J.R., and Kamiguchi, H. (2011). Second messengers and membrane trafficking direct and organize growth cone steering. Nat. Rev. Neurosci. 12, 191-203.

Trotman, L.C., Wang, X., Alimonti, A., Chen, Z., Teruya-Feldstein, J., Yang, H., Pavletich, N.P., Carver, B.S., Cordon-Cardo, C., Erdjument-Bromage, H., et al. (2007). Ubiquitination regulates PTEN nuclear import and tumor suppression. Cell $128,141-156$.

Tsai, L.H., Takahashi, T., Caviness, V.S., and Harlow, E. (1993). Activity and expression pattern of cyclin-dependent kinase 5 in the embryonic mouse nervous system. Development 119, 1029-1040.

Tsai, L.-H., Delalle, I., Caviness, V.S., Chae, T., and Harlow, E. (1994). p35 is a neural-specific regulatory subunit of cyclin-dependent kinase 5. Nature 371, 419423.

Waites, C.L., Craig, A.M., and Garner, C.C. (2005). Mechanisms of vertebrate synaptogenesis. Annu. Rev. Neurosci. 28, 251-274. 
Wang, J., Peng, Q., Lin, Q., Childress, C., Carey, D., and Yang, W. (2010). Calcium activates Nedd4 E3 ubiquitin ligases by releasing the $\mathrm{C} 2$ domain-mediated autoinhibition. J. Biol. Chem. 285, 12279-12288.

Wang, X., Trotman, L.C., Koppie, T., Alimonti, A., Chen, Z., Gao, Z., Wang, J., Erdjument-Bromage, H., Tempst, P., Cordon-Cardo, C., et al. (2007). NEDD4-1 is a proto-oncogenic ubiquitin ligase for PTEN. Cell 128, 129-139.

Welchman, R.L., Gordon, C., and Mayer, R.J. (2005). Ubiquitin and ubiquitin-like proteins as multifunctional signals. Nat. Rev. Mol. Cell Biol. 6, 599-609.

Werner, H.B., Kuhlmann, K., Shen, S., Uecker, M., Schardt, A., Dimova, K., Orfaniotou, F., Dhaunchak, A., Brinkmann, B.G., Möbius, W., et al. (2007). Proteolipid protein is required for transport of sirtuin 2 into CNS myelin. J. Neurosci. 27, 7717-7730.

Wiese, S., Reidegeld, K.A., Meyer, H.E., and Warscheid, B. (2007). Protein labeling by iTRAQ: A new tool for quantitative mass spectrometry in proteome research. PROTEOMICS 7, 340-350.

Wu, D.-C., Yu, Y.-P., Lee, N.T.K., Yu, A.C.H., Wang, J.H.C., and Han, Y.-F. (2000). The expression of Cdk5, p35, p39, and Cdk5 kinase activity in developing, adult, and aged rat brains. Neurochem. Res. 25, 923-929.

Xie, Y., Avello, M., Schirle, M., McWhinnie, E., Feng, Y., Bric-Furlong, E., Wilson, C., Nathans, R., Zhang, J., Kirschner, M.W., et al. (2013). Deubiquitinase FAM/USP9X interacts with the E3 ubiquitin ligase SMURF1 protein and protects it from ligase activity-dependent self-degradation. J. Biol. Chem. 288, 2976-2985.

Xie, Z., Sanada, K., Samuels, B.A., Shih, H., and Tsai, L.-H. (2003). Serine 732 phosphorylation of FAK by Cdk5 is important for microtubule organization, nuclear movement, and neuronal migration. Cell 114, 469-482.

Yamagata, M., Sanes, J.R., and Weiner, J.A. (2003). Synaptic adhesion molecules. Curr. Opin. Cell Biol. 15, 621-632.

Yang, B., Gay, D.L., MacLeod, M.K.L., Cao, X., Hala, T., Sweezer, E.M., Kappler, J., Marrack, P., and Oliver, P.M. (2008a). Nedd4 augments the adaptive immune response by promoting ubiquitin-mediated degradation of Cbl-b in activated $\mathrm{T}$ cells. Nat Immunol 9, 1356-1363.

Yang, L., Wang, S., Sung, B., Lim, G., and Mao, J. (2008b). Morphine induces ubiquitin-proteasome activity and glutamate transporter degradation. J. Biol. Chem. 283, 21703-21713.

Yen, H.-C.S., Xu, Q., Chou, D.M., Zhao, Z., and Elledge, S.J. (2008). Global protein stability profiling in mammalian cells. Science 322, 918-923. 
Yim, E.-K., Peng, G., Dai, H., Hu, R., Li, K., Lu, Y., Mills, G.B., Meric-Bernstam, F., Hennessy, B.T., Craven, R.J., et al. (2009). Rak functions as a tumor suppressor by regulating PTEN protein stability and function. Cancer Cell 15, 304-314.

Yu, Y., Yoon, S.-O., Poulogiannis, G., Yang, Q., Ma, X.M., Villén, J., Kubica, N., Hoffman, G.R., Cantley, L.C., Gygi, S.P., et al. (2011). Phosphoproteomic analysis identifies Grb10 as an mTORC1 substrate that negatively regulates insulin signaling. Science 332, 1322-1326.

Zhang, J., Cicero, S.A., Wang, L., Romito-DiGiacomo, R.R., Yang, Y., and Herrup, K. (2008). Nuclear localization of Cdk5 is a key determinant in the postmitotic state of neurons. Proc. Natl. Acad. Sci.

Zhang, J., Li, H., Zhou, T., Zhou, J., and Herrup, K. (2012). Cdk5 levels oscillate during the neuronal cell cycle. J. Biol. Chem. 287, 25985-25994.

Zhang, P., Yu, P.-C., Tsang, A.H.K., Chen, Y., Fu, A.K.Y., Fu, W.-Y., Chung, K.K., and Ip, N.Y. (2010). S-nitrosylation of cyclin-dependent kinase 5 (Cdk5) regulates its kinase activity and dendrite growth during neuronal development. J. Neurosci. 30, 14366-14370.

Zheng, M., Leung, C.L., and Liem, R.K.H. (1998). Region-specific expression of cyclin-dependent kinase 5 (cdk5) and its activators, p35 and p39, in the developing and adult rat central nervous system. J. Neurobiol. 35, 141-159.

Zou, W., Chen, X., Shim, J.-H., Huang, Z., Brady, N., Hu, D., Drapp, R., Sigrist, K., Glimcher, L.H., and Jones, D. (2011). The E3 ubiquitin ligase WWP2 regulates craniofacial development through mono-ubiquitylation of Goosecoid. Nat Cell Biol $13,59-65$.

Zukerberg, L.R., Patrick, G.N., Nikolic, M., Humbert, S., Wu, C.-L., Lanier, L.M., Gertler, F.B., Vidal, M., Van Etten, R.A., and Tsai, L.-H. (2000). Cables links Cdk5 and $\mathrm{c}-\mathrm{Abl}$ and facilitates $\mathrm{Cdk} 5$ tyrosine phosphorylation, kinase upregulation, and neurite outgrowth. Neuron 26, 633-646. 


\section{CURRICULUM VITAE}

Family Name:

First Name:

Date of Birth:

Gender:

Nationality:

Present address:

E-Mail:

\section{Education}

Apr/2011 - Mar/2015

Sep/2005- Jun/2009

Sep/2009 - Mar/2011

\section{Doctoral Student}

The International Master's/PhD/MD-PhD Neuroscience Program, Faculty of Biology, GeorgAugust-Universität Göttingen, Germany

Hung-En

Oct. $20^{\text {th }} 1986$

Female

Taiwanese

Department of Molecular Neurobiology, Max-Planck-Institute for Experimental Medicine, Hermann-Rein-Str. 3, 37075 Göttingen, Germany hsia@em.mpg.de

Master of Science

The International Master's/PhD/MD-PhD

Neuroscience Program, Faculty of Biology, GeorgAugust-Universität Göttingen, Germany

Bachelor of Science

Department of Biochemical Science and Technology, College of Life Science, National Taiwan University,

Taiwan

\section{Scholarships and Awards}

$\begin{array}{ll}\text { Sep/2009 - Aug/2010 } & \begin{array}{l}\text { Stipend of the Excellence Foundation for the } \\ \text { Promotion of the Max Planck Society, Göttingen, } \\ \text { Germany }\end{array} \\ \text { Jul/2008 - Feb/2009 } & \begin{array}{l}\text { Fellowship for Undergraduate Student Research, } \\ \text { National Science Council, Taiwan }\end{array} \\ 2007 \text { and } 2009 & \begin{array}{l}\text { Presidential Award (Top 5\% of the Department), } \\ \text { National Taiwan University, Taiwan }\end{array}\end{array}$

\section{Research Experiences}

\author{
Apr/2011 - Mar/2015 Doctoral Student \\ 'Roles of the HECT-Type Ubiquitin E3 Ligases of \\ Nedd4 and WWP Subfamilies in Neuron \\ Development' \\ Advisors: Dr. Hiroshi Kawabe/Prof. Dr. Nils Brose \\ Department of Molecular Neuroscience \\ (Head: Prof. Dr. Nils Brose)
}


Max-Planck-Institute for Experimental Medicine, Göttingen, Germany

Oct/2010 - Mar/2011 Master Student

'The Nedd4 Ubiquitin Ligase Subfamily in Rodent Brain Development'

Advisors: Dr. Hiroshi Kawabe/Prof. Dr. Nils Brose Department of Molecular Neuroscience (Head: Prof. Dr. Nils Brose)

Max-Planck-Institute for Experimental Medicine, Göttingen, Germany

May/2010 - Jun/2010 Research Intern

'Roles of Anaphase-Promoting Complex in a Mouse Model of Alzheimer's Disease'

Advisors: Dr. Tanja Kucera/Prof. Dr. Andre Fischer Laboratory for Aging and Cognitive Diseases European Neuroscience Institute, Göttingen, Germany

Mar/2010 - Apr/2010 Research Intern

'Co-localization of AMPA and Kainate Receptors at $C R N \rightarrow P n C$ Synapses'

Advisors: Dr. Kun-Han Lin/Dr. Holger Taschenberger Department of Membrane Biophysics (Head: Prof. Dr. Erwin Neher)

Max Plank Institute for Biophysical Chemistry, Göttingen, Germany

Jan/2010 - Feb/2010 Research Intern

'Optically Activated Neuronal Network'

Advisor: Dr. Ahmed El Hady

Department of Molecular Biology of Neuronal Signals (Head: Prof. Dr. Walter Stühmer)

Max Planck Institute for Experimental Medicine, Göttingen, Germany

Feb/2007 - Jul/2009 Undergraduate Researcher

'Molecular Mechanisms Underlying Stem Cell

Therapy of Alzheimer's Disease'

Advisor: Dr. Kuen-Jer Tsai

Laboratory of Molecular and Cellular Neuroscience

(Principle Investigator: Prof. Che-Kun James Shen) Institute of Molecular Biology, Academic Sinica,

Taipei, Taiwan 


\section{List of Publications}

Kuczera T, Stilling RM, Hsia HE, Bahari-Javan S, Irniger S, Nasmyth K, Sananbenesi F, Fischer A. (2010) The anaphase promoting complex is required for memory function in mice. Learn. Mem. 18:49-57.

Hsia HE, Kumar R, Luca R, Takeda M, Courchet J, Nakashima J, Wu S, Goebbels S, An W, Eickholt BJ, Polleux F, Rotin D, Wu H, Rossner MJ, Bagni C, Rhee JS, Brose N, Kawabe H (2014) Ubiquitin E3 ligase Nedd4-1 acts as a downstream target of PI3K/PTEN-mTORC1 signaling to promote neurite growth. Proc. Nat. Acad. Sci. U.S.A. 111:13205-13210. 